\title{
Percolation on self-dual polygon configurations
}

\author{
Béla Bollobás ${ }^{* \dagger \ddagger} \quad$ Oliver Riordan ${ }^{\S}$
}

May 11, 2010

\begin{abstract}
Recently, Scullard and Ziff noticed that a broad class of planar percolation models are self-dual under a simple condition that, in a parametrized version of such a model, reduces to a single equation. They state that the solution of the resulting equation gives the critical point. However, just as in the classical case of bond percolation on the square lattice, self-duality is simply the starting point: the mathematical difficulty is precisely showing that self-duality implies criticality. Here we do so for a generalization of the models considered by Scullard and Ziff. In these models, the states of the bonds need not be independent; furthermore, increasing events need not be positively correlated, so new techniques are needed in the analysis. The main new ingredients are a generalization of Harris's Lemma to products of partially ordered sets, and a new proof of a type of RussoSeymour-Welsh Lemma with minimal symmetry assumptions.
\end{abstract}

\section{Contents}

1 Introduction

2 The model and results 5

3 A generalization of Harris's Lemma 18

3.1 High probability unions of upsets . . . . . . . . . . . 21

4 Colourings, hypergraphs and crossings 22

4.1 How crossing probabilities vary . . . . . . . . . . 26

* Department of Pure Mathematics and Mathematical Statistics, Wilberforce Road, Cambridge CB3 0WB, UK

${ }^{\dagger}$ Department of Mathematical Sciences, University of Memphis, Memphis TN 38152, USA

$\ddagger$ Research supported in part by NSF grants DMS-0906634, CNS-0721983 and CCF0728928, and ARO grant W911NF-06-1-0076

$\S$ Mathematical Institute, University of Oxford, 24-29 St Giles', Oxford OX1 3LB, UK 
5 A rectangle-crossing lemma 33

5.1 Bond percolation on $\mathbb{Z}^{2} \ldots \ldots \ldots \ldots \ldots$

5.2 A rectangle-crossing lemma for hyperlattices . . . . . . . . . . . 42

5.3 A stronger rectangle-crossing lemma . . . . . . . . . . . 53

6 Self-duality and rectangle crossings 54

7 From rectangle crossings to percolation 63

8 On the critical surface 68

\section{Introduction}

In 1963 Sykes and Essam [30] noticed that, in independent bond percolation, a star with bond probabilities $p_{1}, p_{2}$ and $p_{3}$ may be replaced by a triangle with bond probabilities $r_{1}, r_{2}$ and $r_{3}$, provided the $p_{i}$ and $r_{i}$ satisfy certain equations; in particular, a star in which each bond has probability $p_{0}=1-2 \sin (\pi / 18)$ of being open may be replaced by a triangle with bond probabilities $1-p_{0}$. Sykes and Essam went on to use this star-triangle transformation to predict that $p_{0}$ and $1-p_{0}$ should be the critical probabilities for bond percolation on the hexagonal and triangular lattices, respectively. In 1981 Wierman 31. gave a rigorous proof of this result with the aid of a Russo-Seymour-Welshtype theorem. In 1982 Kesten [17] extended Wierman's theorem to describe the 'critical surface' of weighted bond percolation on the triangular lattice, although the details were worked out only in 1999 by Grimmett 13. Later, in 2008, it was shown 7 that the sum of the critical probabilities of a centrally symmetric planar lattice and its dual is 1; more generally, an analogous statement allowing for percolation with different probabilities for different bonds was proved, giving the Kesten-Grimmett theorem as an easy corollary.

In 1984 Wierman 32 used the general star-triangle transformation to determine the exact critical probability for bond percolation (with equal bond probabilities) on a lattice obtained from the square lattice by adding some diagonals. Using a simpler transformation, Suding and Ziff [29] deduced the critical probability for site percolation on the extended Kagomé lattice from Wierman's result for the hexagonal lattice.

Recently, extending work of Scullard 24 and Chayes and Lei 10, Ziff 35 and Ziff and Scullard [36 proposed a simple criterion predicting the value of the critical probability for a wide variety of percolation models in the plane. In addition to the usual independent site and/or bond percolation models on a number of lattices, these models include cases which can be seen as bond percolation with local dependencies between the states of certain bonds.

The predictions of Scullard and Ziff are similar in nature to those of Sykes and Essam [30] mentioned above: having shown that for a certain probability $p_{0}$ the percolation model is 'self-dual', they state that this probability $p_{0}$ is therefore critical. Mathematically, there is a folklore 'conjecture' (with, as far 
as we are aware, no precise formulation) stating that any 'reasonable' self-dual planar model is critical. This conjecture is still wide open. It is well known to hold in certain special cases, in particular for site or bond percolation on lattices with certain symmetries, such as reflection in a line (see Kesten [17]), or rotational symmetry of any order, as shown in 7]. More generally, as remarked in [7, it can be shown for site or bond percolation on any lattice by combining results of Sheffield [27, Corollary 9.4.6], Aizenman, Kesten and Newman [1] and Menshikov [21].

Chayes and Lei [10] independently described a special case of the ScullardZiff criterion (as well as a generalization to random cluster models), and gave a sketch proof of criticality under an extra assumption. In a recent preprint, Wierman and Ziff 33 proved criticality in certain special cases, using known results on self-dual planar lattices.

In this paper we shall prove that the Scullard-Ziff criterion does indeed give the critical point for a wide variety of planar percolation models. In the original papers in the physics literature, the exact scope of applicability of the criterion is not entirely clear. In this paper we shall define precisely a general class of models that are self-dual in the appropriate sense, and use new methods to show that the self-dual point is indeed critical in all cases.

Although full definitions will be given only in the next section, let us illustrate some simple special cases of our main result, starting with one very concrete (but rather specific) example, and then turning to a more general family.

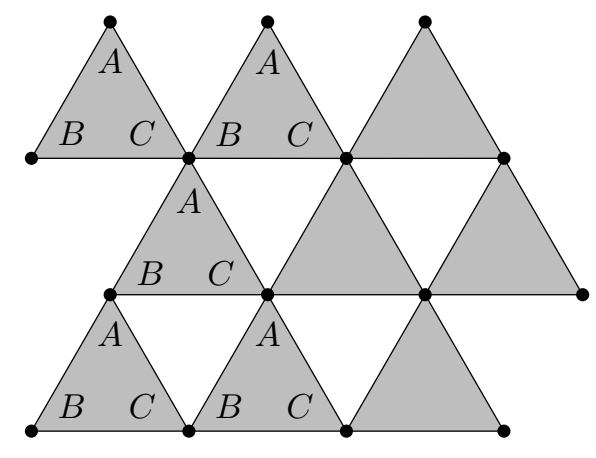

Figure 1.1: The triangular lattice, with alternate triangles shaded.

Example 1.1. Consider the usual triangular lattice shown in Figure 1.1. Given a parameter $0<p<1$, we initially select each bond (i.e., edge) independently with probability $p$. But then bonds within a shaded triangle 'compete': if there are two bonds, the first in the clockwise order 'wins', and the other is deleted. However, if all three bonds are present there is a standoff, and all three remain. This results in a configuration of bonds such that, within each shaded triangle, with probability $(1-p)^{3}$ no bonds are present, with probability $p^{3}$ all three are present, and otherwise exactly one bond is present, with each of these cases 
having probability $\frac{1}{3}\left(1-p^{3}-(1-p)^{3}\right)=p(1-p)$. The configurations in different triangles are of course independent.

Applying our main results to this particular model we shall see that (with probability 1) the remaining bonds form a graph containing an infinite component if and only if $p>1 / 2$; in other words, the model percolates if and only if $p>1 / 2$. Note for later that considering a single shaded triangle, the probability that the vertices $A$ and $B$ are connected within this triangle is $p(1-p)+p^{3}$, as is the probability that $B$ and $C$ are connected. The probability that both events hold is $p^{3}$, since this happens if and only if all three bonds are selected.

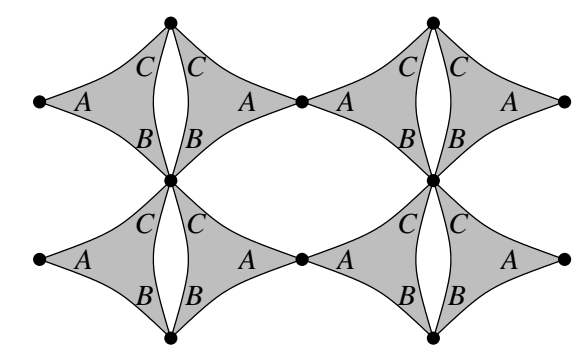

Figure 1.2: A lattice of labelled triangles with no axes of symmetry, and no rotational symmetries.

Example 1.2. More generally, consider, for example, either the usual triangular lattice as above (viewed as an arrangement of shaded triangles), or the lattice of triangles a small part of which is illustrated in Figure 1.2. These are both examples of self-dual hyperlattices, to be defined in the next section. Suppose that each shaded triangle contains some mechanism that connects certain subsets of its vertices, with these processes independent in different triangles; we have given one example above, but the mechanism is irrelevant, only the final connection probabilities matter. Suppose that in each triangle the probability that all vertices are connected (inside the triangle) is $p_{A B C}$, that none are connected is $p_{\emptyset}$, that $A$ and $B$ are connected to each other but not to $C$ is $p_{A B}$, and so on, with these probabilities the same for all triangles. Then, except possibly in the degenerate case $p_{A B C}=p_{\emptyset}=0$, there is (with probability 1 ) an infinite connected component if and only if $p_{A B C}>p_{\emptyset}$.

Even the very special cases described above are outside the scope of existing results, for several reasons. Firstly, in general they do not correspond to independent bond or site percolation on any lattice (as required in [33]). This is certainly the case when increasing events are not positively correlated; see the discussion in Section 2. Such correlation is absent in Example 1.1 when $p$ is equal to or close to $1 / 2$, since $p^{3}<\left(p(1-p)+p^{3}\right)^{2}$ when $p=1 / 2$. Positive correlation is also required for the arguments in [10. Secondly, in Example 1.2 there is no rotational or reflectional symmetry, as required in [33] and in [10], so existing Russo-Seymour-Welsh-type results do not apply. 
Our results show that self-duality implies criticality in a context that is much broader than the Scullard-Ziff criterion; an example is given in Figure 2.7.

Mathematically, the main interest of this paper is perhaps in the development of new tools needed to analyze the general model, including new proofs of analogues of the Russo-Seymour-Welsh Lemma, and a generalization of Harris's Lemma. The rest of the paper is organized as follows. In Section 2 we describe the model we shall study, and state our main results. In Section 3 we present a generalization of Harris's Lemma to products of posets. In Section 4 we prove various technical results about the model. The heart of the paper is Section 5. where we prove a new RSW-type lemma; the proof is rather involved, so we first illustrate the ideas in a simpler setting (bond percolation on $\mathbb{Z}^{2}$ ) in Subsection 5.1. In Section 6 we show how to apply this lemma using only the minimal symmetry guaranteed by self-duality. Finally, in Section 7 we show that (as in 4), using a suitable sharp-threshold result, it is but a small step from rectangle crossings to the final results. In Section 8 we conclude with a brief discussion of the behaviour of the model in the critical case.

\section{The model and results}

The starting point of our investigation is an embedding of a suitable hypergraph in the plane, as described implicitly by Ziff and Scullard [36] and explicitly by Wierman and Ziff [33. In these papers the hypergraphs considered are 3uniform, but much of the time there is no need for this restriction. Since the concept of a plane hypergraph seems fundamental, we give several equivalent definitions. To avoid irrelevant difficulties we always assume piecewise linearity of all curves.

By a polygon we mean a piecewise-linear closed curve $P$ in the plane that, if it touches itself at all, does so only externally at some vertices. (To be pedantic, $P$ is the image of some regular $n$-gon $P^{\prime}$ under a piecewise linear map defined on the closed domain bounded by $P^{\prime}$ that is injective except possibly at the vertices of $P^{\prime}$.) Such a polygon surrounds (more precisely winds around) a simply connected open set that we call its interior.

By a plane hypergraph $\mathcal{H}$ we mean a set of points of $\mathbb{R}^{2}$, the vertices, together with a set of polygons, the hyperedges, with the following properties:

(i) any bounded subset of the plane contains only finitely many vertices, and meets only finitely many hyperedges,

(ii) the interiors of the hyperedges are disjoint,

(iii) each hyperedge is incident with at least one vertex, and

(iv) hyperedges meet themselves or each other only at vertices.

Note that we allow a hyperedge to meet the same vertex several times, as in Figure 2.1 simply because there turns out to be no reason not to. Property (i) ensures that each vertex meets only finitely many hyperedges, and vice versa. 
In this paper, all plane hypergraphs we consider are connected, in the natural sense.
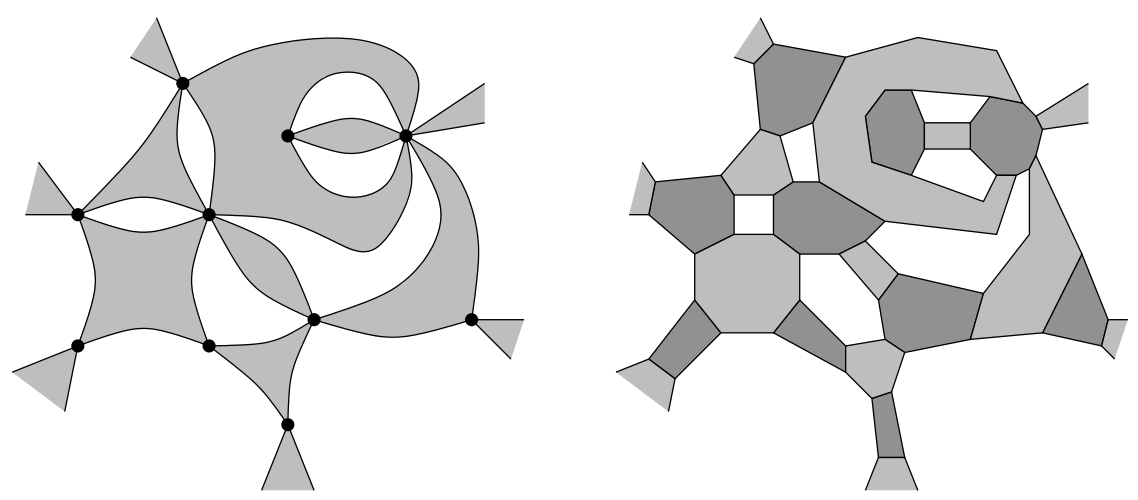

Figure 2.1: On the left is part of a plane hypergraph $\mathcal{H}$ : the shaded regions are the (interiors of the) polygons corresponding to hyperedges. The same picture may be seen as a proper 2-colouring of the faces of a plane graph. On the right is the corresponding 3-coloured cubic map. Note that not all edges are drawn as straight lines (see the top right of the figure).

Two plane hypergraphs are isomorphic if there is a homeomorphism from the plane to itself mapping one into the other, in the obvious sense. Of course, given a plane hypergraph (or indeed an isomorphism class of plane hypergraphs) there is a corresponding abstract hypergraph; more precisely, a (multi-)hyper(multi-)graph, where we have included 'multi' twice to indicate that two or more hyperedges may be incident with the same set of vertices, and a single hyperedge may be incident with a vertex more than once. However, we shall work directly with the drawings throughout.

Plane hypergraphs are natural generalizations of plane (multi)graphs. Indeed, we may think of a plane graph as a representation of an abstract graph, with the vertices represented by points, and the edges by connected sets meeting only at the vertices. The edges of a graph are naturally represented by minimal sets connecting the corresponding vertices, i.e., simple curves, but in the hypergraph case polygons are more natural, so we use them even for hyperedges with only two vertices (or indeed, one vertex). When we come to percolation in a moment, the idea is that instead of each edge in a graph randomly either connecting its vertices or not, independently of the other edges, each hyperedge will randomly connect some subsets of its vertices, independently of the other hyperedges.

It is easy to check that (connected, as always in this paper) plane hypergraphs $\mathcal{H}$ correspond exactly to shaded locally finite connected plane multigraphs $G$, where the faces of $G$ are properly coloured grey and white, so that 
every edge borders faces of different colours, with any unbounded faces white. (At this stage there may be one or more unbounded faces; in the bulk of the paper all faces will be bounded.) Indeed, vertices correspond to vertices, and the hyperedges of $\mathcal{H}$ are simply the grey faces of $G$, as on the left in Figure 2.1 Of course, a plane graph $G$ with at most one unbounded face has an appropriate shading if and only if every degree is even, and then it has either one or two shadings, depending on whether or not it has an unbounded face.

Note that if $e$ is a hyperedge incident with $|e|$ vertices (counting multiplicity), then, as a polygon, $e$ is made up of $|e|$ segments joining vertices, corresponding to the edges of $G$. We cannot in general draw these segments as straight lines. For example, $\mathcal{H}$ may contain triples $u v w$ and $u v w^{\prime}$, say; furthermore, there may be further hyperedges inside the region bounded by the two curves joining $u$ and $v$ associated to these triples.

By a face of a plane hypergraph $\mathcal{H}$ (defined as above) we mean a component of what is left of the plane after removing all hyperedges and their interiors, i.e., a white face of the corresponding graph $G$. For our next few definitions (in particular that of duality) to make sense, it is convenient to insist that each face of $\mathcal{H}$ has finitely many edges in its boundary. This is equivalent to imposing the condition that if $\mathcal{H}$ is infinite, then it has no unbounded faces. (Of course, if $\mathcal{H}$ is finite, then it necessarily has exactly one unbounded face.) When it comes to percolation, we naturally consider only infinite $\mathcal{H}$.

There is yet another way of defining plane hypergraphs, which will turn out to be much more convenient to work with, but is at first sight perhaps less natural (at least for percolation). Given a (connected, as usual) plane hypergraph $\mathcal{H}$ and the corresponding graph $G$ as above, replace each vertex $v$ of $\mathcal{H}$ by a black $2 d$-gon, where $d=d(v)$ is the degree of $v$, each face by a white $2 d$-gon, where $d$ is the number of edges (of $G$ ) bounding the face, and each hyperedge $e$ incident with $d$ vertices (counted with multiplicity) by a grey $2 d$-gon. In this way we obtain a cubic planar map $M$ in which the faces are properly coloured black, white and grey; see Figure 2.1 (As before, we cannot necessarily draw the edges of the polygons as straight lines.)

The reverse transformation is even simpler: starting from a cubic map $M$ (i.e., a connected locally finite 3 -regular plane graph in which each face has finitely many edges in its boundary) in which the faces are properly coloured black, white and grey, we simply contract each black face to a point to form a vertex of $\mathcal{H}$, and take the grey faces to form the hyperedges. In what follows we shall refer to such a coloured map $M$ as a map hypergraph (or simply a hypergraph, when there is no danger of confusion), and denote it also by $\mathcal{H}$.

Note that when $\mathcal{H}$ is a graph (i.e., $\mathcal{H}$ is 2 -uniform), then the corresponding map is the one considered in Chapter 3 of [6] (see Figures 2 and 4, for example). There, the 4-gon corresponding to an edge is coloured black or white according to whether the edge is open or closed; here, the $2|e|$-gon corresponding to a hyperedge $e$ is grey for now, but will be coloured with a mixture of black and white later.

By a plane hyperlattice $\mathcal{H}$ we shall mean an infinite connected plane hypergraph (defined in any of the three ways above) with a lattice $\mathcal{L}$ of translational 
symmetries, i.e., such that there are linearly independent vectors $\mathbf{a}$ and $\mathbf{b}$ with the property that translation of the plane through either vector maps the drawing into itself in the obvious sense, corresponding to an isomorphism of the underlying hypergraph. Throughout, we view $\mathcal{L}=\{m \mathbf{a}+n \mathbf{b}: m, n \in \mathbb{Z}\}$ as a subset of $\mathbb{R}^{2}$. More formally, we define a plane hyperlattice to be a pair $(\mathcal{H}, \mathcal{L})$ as above, since in what follows $\mathcal{L}$ need not be the full lattice of translational symmetries of $\mathcal{H}$; in spite of this, we usually omit $\mathcal{L}$ from the notation. Naturally, when we consider isomorphisms of plane hyperlattices, these are required to preserve the corresponding lattices of symmetries. More precisely, a homeomorphism $S: \mathbb{R}^{2} \rightarrow \mathbb{R}^{2}$ is an isomorphism from the plane hyperlattice $(\mathcal{H}, \mathcal{L})$ to $\left(\mathcal{H}^{\prime}, \mathcal{L}^{\prime}\right)$ if it corresponds to a plane hypergraph isomorphism and satisfies $S(x+\ell)=S(x)+T(\ell)$ for all $x \in \mathbb{R}^{2}$ and $\ell \in \mathcal{L}$, where $T$ is a linear map with $T(\mathcal{L})=\mathcal{L}^{\prime}$.

In the context of percolation, the natural notion of the dual of a plane hypergraph $\mathcal{H}$ turns out to be the plane hypergraph $\mathcal{H}^{*}$ defined as follows. Take a vertex of $\mathcal{H}^{*}$ inside each face of $\mathcal{H}$. To obtain the hyperedges of $\mathcal{H}^{*}$, replace each hyperedge $e$ of $\mathcal{H}$ by the dual hyperedge $e^{*}$ joining the vertices corresponding to the faces that $e$ meets, as in Figure 2.2. In the special case of 2 -uniform hyper-

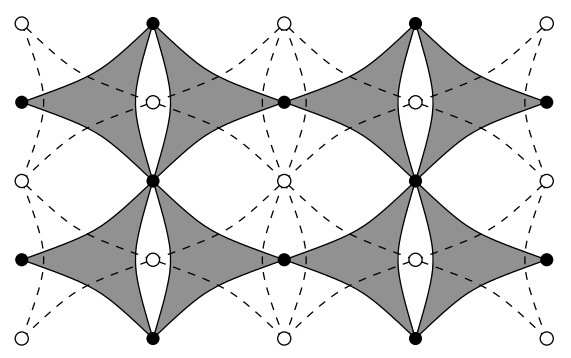

Figure 2.2: Part of a 3-uniform plane hyperlattice (filled circles and shaded triangles) and its dual (open circles and dashed triangles). The hyperedges of the dual are the concave dashed triangles.

graphs, i.e., graphs, this is the usual notion of planar duality. In the 3-uniform case considered (with mild additional restrictions) by Wierman and Ziff 33, the notion of duality just defined coincides with theirs; the description of Ziff and Scullard 36] seems to be ambiguous.

In terms of the shaded graph $G$, the operation of taking the dual is rather complicated - it is not simply reversing the shading. However, in the 3 -coloured map formulation, it is very simple to construct the dual: simply exchange black and white. Indeed, one can think of a hyperedge and its dual together as corresponding to a grey face of the map $M$ (for example, in Figure 2.2 one can take the intersection of $e$ and $e^{*}$ as the grey face); the vertices of $\mathcal{H}$ correspond to the black faces of $M$, and the vertices of $\mathcal{H}^{*}$ to the white faces of $M$.

Of course, choosing the drawing appropriately, we may take the dual $\mathcal{H}^{*}$ of a plane hyperlattice $\mathcal{H}$ to be a plane hyperlattice, and the dual of $\mathcal{H}^{*}$ to be $\mathcal{H}$. A plane hyperlattice $\mathcal{H}$ is self-dual if $\mathcal{H}^{*}$ is isomorphic to $\mathcal{H}$; examples are shown 
in Figures 2.2, 2.5 and 2.7.

If $P$ is a polygon in the plane, then by a non-crossing partition of its vertex set $V(P)$ we mean a partition $\pi$ such that no two distinct parts of $\pi$ contain interlaced pairs of vertices: if $x, y, z, w$ are four distinct vertices appearing in this cyclic order around $P$, a non-crossing partition $\pi$ cannot contain two parts one of which includes $x$ and $z$, and the other $y$ and $w$. Equivalently, a partition $\pi$ of $V(P)$ is a non-crossing partition if and only if it may be realized by constructing disjoint (path-)connected subsets $S_{i}$ of $P$ (which we take to include its interior) so that each part of $\pi$ is the intersection of some $S_{i}$ with $V(P)$.

The dual $\pi^{*}$ of a non-crossing partition $\pi$ is the non-crossing partition of the edges of $P$ in which two edges $e$ and $f$ are in the same part if and only if they are not interlaced with two vertices $x$ and $y$ in a common part of $\pi$. Here interlaced means that the edges and vertices occur in the cyclic order $e, x, f, y$ or its reverse.

Turning finally to percolation, the state of a hyperedge $e$ will simply be a non-crossing partition of its vertices. (More precisely, of the incidences of $e$ with its vertex set, so if $e$ touches itself, the relevant vertex appears multiple times in the groundset of the partition.) A configuration $\omega$ is an assignment of a state to each hyperedge of the hypergraph $\mathcal{H}$ under consideration. We think of the state of a hyperedge $e$ as describing connections within $e$. In particular, by an open path in a configuration $\omega$ we mean a sequence $v_{0} e_{1} v_{1} e_{2} \ldots e_{\ell} v_{\ell}$ such that, for each $i$, the partition of the vertices of $e_{i}$ has a part containing both $v_{i-1}$ and $v_{i}$. Two vertices are connected in $\omega$ if they are joined by an open path, and the open clusters of $\omega$ are the maximal connected sets of vertices.

Finally, a hyperlattice percolation model consists of a plane hyperlattice $(\mathcal{H}, \mathcal{L})$ together with a probability measure on configurations on $\mathcal{H}$ such that the states of different hyperedges are independent, and the measure is preserved by the action of $\mathcal{L}$. In other words, if $e^{\prime}$ is a translate of $e$ under an element of $\mathcal{L}$, then corresponding states in $e^{\prime}$ and $e$ have the same probabilities. Note that for a single hyperedge $e$, all probability distributions on the set of non-crossing partitions associated to $e$ are allowed.

As usual, the sigma-field of measurable events is the one generated by cylindrical sets, i.e., by events depending only on the states of a finite set of hyperedges. In fact, except when defining percolation, throughout this paper we can work with large enough finite regions of $\mathcal{H}$, so there are no issues of measurability.

The dual $\omega^{*}$ of a configuration $\omega$ on $\mathcal{H}$ is the configuration on $\mathcal{H}^{*}$ in which the state of $e^{*}$ is the dual of the state of $e$ (noting that vertices of $e^{*}$ correspond to edges of the polygon $e$ ). It is not hard to check that finite open clusters in $\omega$ are surrounded by open cycles in $\omega^{*}$ and vice versa; this is most easily seen in the colouring formulation described at the start of Section 4 .

Given a plane hyperlattice $(\mathcal{H}, \mathcal{L})$, suppressing $\mathcal{L}$ in the notation as usual, the hyperlattice percolation models on $\mathcal{H}$ may be parametrized as follows. First pick one representative $e_{i}$ of each orbit of the action of $\mathcal{L}$ on the hyperedges. Then for each non-crossing partition $\pi$ of the vertices of $e_{i}$, choose a probability 
$p_{i, \pi}$, subject only to $\sum_{\pi} p_{i, \pi}=1$ for each $i$. We call such a vector $\mathbf{p}=\left(p_{i, \pi}\right)_{i, \pi}$ a probability vector (for $\mathcal{H}$ ), and write $\mathcal{H}(\mathbf{p})$ for the corresponding percolation model. The dual vector $\mathbf{p}^{*}$ assigns the probability $p_{i, \pi}$ to the partition $\pi^{*}$ of $e_{i}^{*}$, so $\mathcal{H}^{*}\left(\mathbf{p}^{*}\right)$ is a hyperlattice percolation model on $\mathcal{H}^{*}$.

The hyperlattice percolation model $\mathcal{H}(\mathbf{p})$ is self-dual if $\mathcal{H}(\mathbf{p})$ and $\mathcal{H}^{*}\left(\mathbf{p}^{*}\right)$ are isomorphic, i.e., if there is an isomorphism from $\mathcal{H}$ to $\mathcal{H}^{*}$ such if $e \in E(\mathcal{H})$ and $f \in E\left(\mathcal{H}^{*}\right)$ correspond under the isomorphism, then each partition $\pi$ of $f$ has the same probability in $\mathcal{H}^{*}\left(\mathbf{p}^{*}\right)$ as the corresponding partition of $e$ does in $\mathcal{H}(\mathbf{p})$. Our aim is to show that self-dual hyperlattice models are 'critical', but first we must define what critical means.

The set of partitions of a (here finite) set $S$ forms a poset $\mathcal{P}$ in a natural way: we have $\pi \preccurlyeq \pi^{\prime}$ if any two elements in the same part of $\pi$ are in the same part of $\pi^{\prime}$, i.e., the parts of $\pi^{\prime}$ are unions of those of $\pi$, i.e., if $\pi$ refines $\pi^{\prime}$.

Given a hyperedge $e_{i}$ as above, let $\mathcal{P}=\mathcal{P}_{e_{i}}$ be the poset formed by the non-crossing partitions of the vertices of $e_{i}$. An upset $\mathcal{U}$ in $\mathcal{P}$ is a subset of $\mathcal{P}$ such that if $\pi \in \mathcal{U}$ and $\pi \prec \pi^{\prime}$ then $\pi^{\prime} \in \mathcal{U}$. Given an upset $\mathcal{U}$ in $\mathcal{P}_{e_{i}}$ and a probability vector $\mathbf{p}$, let $p_{i}(\mathcal{U})=\sum_{\pi \in \mathcal{U}} p_{i, \pi}$ denote the probability that the state of $e_{i}$ is in $\mathcal{U}$. Given two probability vectors $\mathbf{p}$ and $\mathbf{q}$, we say that $\mathbf{q}$ dominates $\mathbf{p}$ if $q_{i}(\mathcal{U}) \geq p_{i}(\mathcal{U})$ for each $i$ and each upset $\mathcal{U} \subset \mathcal{P}_{e_{i}}$. We say that $\mathbf{q}$ strictly dominates $\mathbf{p}$, and write $\mathbf{q} \succ \mathbf{p}$, if $q_{i}(\mathcal{U})>p_{i}(\mathcal{U})$ for each $i$ and each non-trivial upset $\mathcal{U} \subset \mathcal{P}_{e_{i}}$, i.e., for all upsets apart from $\mathcal{U}=\emptyset$ and $\mathcal{U}=\mathcal{P}_{e_{i}}$. Note that we can have $\mathbf{p} \neq \mathbf{q}$ such that $\mathbf{q}$ dominates $\mathbf{p}$ but does not strictly dominate it.

Hall's theorem implies that $\mathbf{q}$ dominates $\mathbf{p}$ if and only if $\mathbf{q}$ can be obtained from $\mathbf{p}$ by moving 'probability mass' from elements $p_{i, \pi}$ to elements $p_{i, \pi^{\prime}}$ with $\pi \prec \pi^{\prime}$. In the case of strict domination, we can assume that, for each $i$, a non-zero mass is moved from each $\pi$ to each $\pi^{\prime} \succ \pi$.

A percolation model $\mathcal{H}(\mathbf{p})$ percolates if the probability that the open cluster containing any given vertex is infinite is positive. As usual, this is equivalent to the existence with probability 1 of an infinite open cluster. The model $\mathcal{H}(\mathbf{p})$ is critical if two conditions hold: for any $\mathbf{q} \succ \mathbf{p}$ the model $\mathcal{H}(\mathbf{q})$ percolates, and for any $\mathbf{q} \prec \mathbf{p}$ the model $\mathcal{H}(\mathbf{q})$ does not percolate.

We say that the model $\mathcal{H}(\mathbf{p})$ exhibits exponential decay (of the volume) if there is a constant $\alpha>0$ such that for any fixed vertex $v$ the probability that the open cluster containing $v$ contains at least $n$ vertices is at most $e^{-\alpha n}$ for all $n \geq 2$. Our main result is the following.

Theorem 2.1. Let $\mathcal{H}(\mathbf{p})$ be a self-dual hyperlattice percolation model. Then for any $\mathbf{q} \succ \mathbf{p}$ the model $\mathcal{H}(\mathbf{q})$ percolates, and for any $\mathbf{q} \prec \mathbf{p}$ the model $\mathcal{H}(\mathbf{q})$ exhibits exponential decay. In particular, $\mathcal{H}(\mathbf{p})$ is critical.

Although this is far from the main point, a very special case is that the critical probability for bond percolation on any self-dual planar lattice is $1 / 2$. Note that the condition $\mathbf{q} \succ \mathbf{p}$ is stronger than what one could hope for, namely a similar result with this condition replaced by $\mathbf{q}$ dominating $\mathbf{p}$ and $\mathbf{q} \neq \mathbf{p}$. However, one would then need to rule out degenerate special cases (corresponding to increasing the probability of bonds that are 'dead ends' in a bond percolation 
model, for example). Also, Theorem 2.1 with the present conditions is strong enough for the main application, Corollary 2.3 below.

It turns out that in proving Theorem 2.1] we do not require an exact isomorphism between $\mathcal{H}(\mathbf{p})$ and $\mathcal{H}^{*}\left(\mathbf{p}^{*}\right)$. We call two hyperlattice percolation models $\mathcal{H}_{1}\left(\mathbf{p}_{1}\right)$ and $\mathcal{H}_{2}\left(\mathbf{p}_{2}\right)$ equivalent if they can be coupled so that, for some constant $C$, for every open path $P$ in either model there is an open path $P^{\prime}$ in the other model at Hausdorff distance at most $C$ from $P$. Roughly speaking, the typical reason for two models to be equivalent is that they can be viewed as different ways of realizing a single underlying model.

For example, consider a plane triangulation $G$ with a lattice $\mathcal{L}$ of translational symmetries, such as the triangular lattice. Then there are two natural ways to form a hyperlattice from $G$, illustrated (for a more complicated lattice) in Figure 2.3. In the first, there is a vertex for each edge of $G$, in the second,
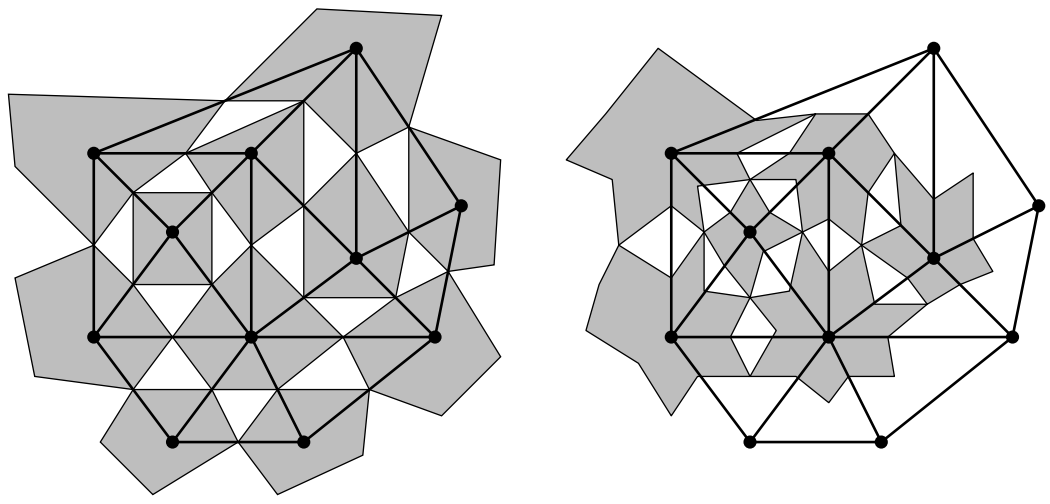

Figure 2.3: The filled circles and thick lines show part of a planar triangulation $G$, which is assumed to have a lattice of symmetries not visible on this scale. On the left is part of the hyperlattice $\mathcal{H}$ formed from $G$ by taking a vertex for each edge, and a hyperedge for each vertex. On the right is part of the hyperlattice $\mathcal{H}^{\prime}$ formed by taking a vertex for each face of $G$, and a hyperedge for each vertex. Note that $\mathcal{H}$ and $\mathcal{H}^{\prime}$ are dual as hyperlattices.

a vertex for each face of $G$. In either case, there is a hyperedge for each vertex $v$ of $G$; this hyperedge is incident to all vertices corresponding to edges or faces of $G$ that $v$ is incident to. Within each hyperedge, assign probability $p$ to the partition in which all vertices are in a single part, and $1-p$ to that in which every vertex is in a separate part. Let us call a hyperedge open if we select the partition into one part, and closed otherwise. Then the resulting models $\mathcal{H}(p)$ and $\mathcal{H}^{\prime}(p)$ are equivalent using the natural coupling, i.e., the coupling in which the hyperedges in the two models corresponding to a vertex $v$ of $G$ have the same state, open or closed. Indeed, in either model an open path of length more than 1 consists of a sequence of open hyperedges with consecutive ones sharing (hyperlattice) vertices. But two hyperedges share a vertex in $\mathcal{H}$ if and only if 
the corresponding vertices of $G$ are joined by an edge, and share a vertex in $\mathcal{H}^{\prime}$ if and only if the corresponding vertices of $G$ are in a common face. In a triangulation, two vertices are in a common face if and only if they are joined by an edge.

Emphasizing the lattice in the notation, for a change, we say that a hyperlattice percolation model $(\mathcal{H}(\mathbf{p}), \mathcal{L})$ is approximately self-dual if there is a model $\left(\mathcal{H}^{\prime}\left(\mathbf{p}^{\prime}\right), \mathcal{L}\right)$ equivalent to $\left(\mathcal{H}^{*}\left(\mathbf{p}^{*}\right), \mathcal{L}\right)$ such that $\left(\mathcal{H}^{\prime}\left(\mathbf{p}^{\prime}\right), \mathcal{L}\right)$ and $(\mathcal{H}(\mathbf{p}), \mathcal{L})$ are isomorphic as plane hyperlattices, with the corresponding linear map $T$ an isometry of the plane. The last restriction is a technicality: in the case of (exact) self-duality we did not impose it, but as we shall see in Lemma 6.7. any isomorphism witnessing self-duality has this property (after a suitable affine transformation). This is presumably also true for approximate self-duality, but as the condition will (we believe) self-evidently hold in any applications, we do not bother checking this. For our proofs, approximate self-duality is (apart from one technicality) just as good as self-duality, so we obtain the following strengthening of Theorem 2.1. In this result 'malleability' is a technical condition we shall introduce later (see Definitions 5.8 and 5.9); in the 3-uniform case, all hyperlattice percolation models are malleable. Also, any 'site percolation' model, where only the partition into singletons and that into a single part occur, is malleable.

Theorem 2.2. Let $\mathcal{H}(\mathbf{p})$ be a malleable approximately self-dual hyperlattice percolation model. Then for any $\mathbf{q} \succ \mathbf{p}$ the model $\mathcal{H}(\mathbf{q})$ percolates, and for any $\mathbf{q} \prec \mathbf{p}$ the model $\mathcal{H}(\mathbf{q})$ exhibits exponential decay. In particular, $\mathcal{H}(\mathbf{p})$ is critical.

To indicate that this extension may be useful, consider a plane triangulation $G$ with a lattice of translational symmetries, and a real number $0<p<1$. Consider the two hyperlattice percolation models $\mathcal{H}(p)$ and $\mathcal{H}^{\prime}(p)$ corresponding to site percolation on $G$, defined as above. Then $\mathcal{H}$ and $\mathcal{H}^{\prime}$ are dual to each other, so $\mathcal{H}(p)$ and $\mathcal{H}^{\prime}(1-p)$ are dual as hyperlattice percolation models. Since, as noted above, $\mathcal{H}(p)$ and $\mathcal{H}^{\prime}(p)$ are equivalent, we see that $\mathcal{H}(1 / 2)$ is approximately self-dual, so Theorem 2.2 implies that the critical probability for site percolation on $G$ is $1 / 2$. In itself this is not new (see the discussion in the introduction), but it indicates that the models to which Theorem 2.2 applies include ones with a site percolation 'flavour'.

We have just seen that site percolation on a triangulation with a lattice of symmetries, which for $p=1 / 2$ is easily seen to be self-dual in an appropriate sense, may be transformed to a hyperlattice percolation model that is only approximately self-dual. In this case, the duality is clearer in the site percolation formulation than the hyperlattice one. Unsurprisingly, there are also cases where the reverse holds. Indeed, for any plane hyperlattice $\mathcal{H}$, selecting only the partitions in which all vertices in a given edge are connected or none are, we obtain a site percolation model on an (in general non-planar) graph. For example, taking $\mathcal{H}$ as in Figure 1.2. one obtains the non-planar graph in Figure 2.4 Theorem 2.1] shows that if $\mathcal{H}$ is self-dual, then the critical probability for site percolation on the resulting graph is $1 / 2$. 


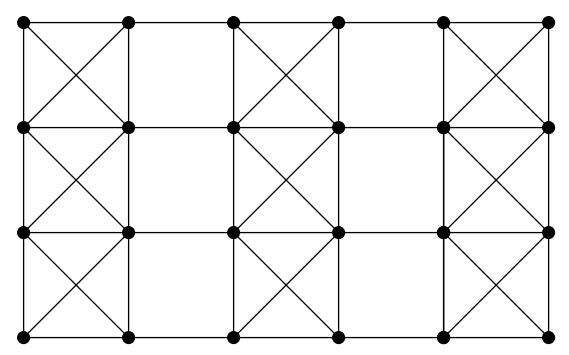

Figure 2.4: A non-planar lattice which is 'self-dual' for site percolation.

Returning to the original motivation, the key observation of Ziff [35] and Ziff and Scullard [36] (present also in the original papers of Scullard 24] and Chayes and Lei [10] in the special case where $\mathcal{H}$ is the 'triangular hyperlattice' $T$ shown in Figure 2.5) is that if $\mathcal{H}$ is 3 -uniform, then the condition for self-duality becomes very simple, at least if one takes the same connection probabilities in all triangles. Unfortunately, this involves some further definitions, to allow for non-symmetric cases.

By a labelled plane hyperlattice we mean a plane hyperlattice in which the vertices around each hyperedge are labelled $1,2, \ldots, k$ in a way that is consistent with either the clockwise or anti-clockwise cyclic order within each hyperedge, and is globally consistent with the lattice $\mathcal{L}$ of translational symmetries. When $k=3$ or $k=4$ we often use letters $A, B, \ldots$, to denote the labels rather than numbers. In the 3 -uniform case, assigning labels as above amounts to designating the vertices of a hyperedge $e$ as its $A$-, $B$ - and $C$-vertex in any order, as in 33 . The simplest example of a labelled plane hyperlattice is the labelled triangular hyperlattice shown in Figure 2.5. Another example is illustrated in Figure 2.6.

Note that the $A$-vertex of one hyperedge may be the $B$-vertex of another hyperedge (or indeed, of the same hyperedge if it touches itself). Given a $k$-uniform labelled plane hyperlattice and a probability vector $\mathbf{p}$ consisting of probabilities $p_{\pi}, \pi \in \mathcal{P}_{\{1,2, \ldots, k\}}$, that sum to 1 , there is a corresponding hyperlattice percolation model $\mathcal{H}(\mathbf{p})$ : for every hyperedge $e$, we assign $p_{\pi}$ as the probability that the vertices of $e$ are partitioned according to $\pi$, with the groundset $\{1,2, \ldots, k\}$ of $\pi$ corresponding to the vertices of $e$ in a manner indicated by the labelling.

Specializing to the 3 -uniform case, the dual $e^{*}$ of an edge $e$ inherits a labelling from $e$ : take the first vertex to be the one opposite the first vertex of $e$, and so on, so a labelled 3 -uniform plane hyperlattice $\mathcal{H}$ has a dual $\mathcal{H}^{*}$ that is again a labelled 3-uniform plane hyperlattice.

If $A c B a C b$ is a triangle with vertices $A, B, C$ and edges $a, b$ and $c$, then there are five possible partitions of the vertex set, all of which are non-crossing. These are represented by Wierman and Ziff [33] as $A|B| C, A B|C, A C| B, B C \mid A$, and $A B C$; we use the more compact notation $\emptyset, A B, A C, B C$ and $A B C$. The 


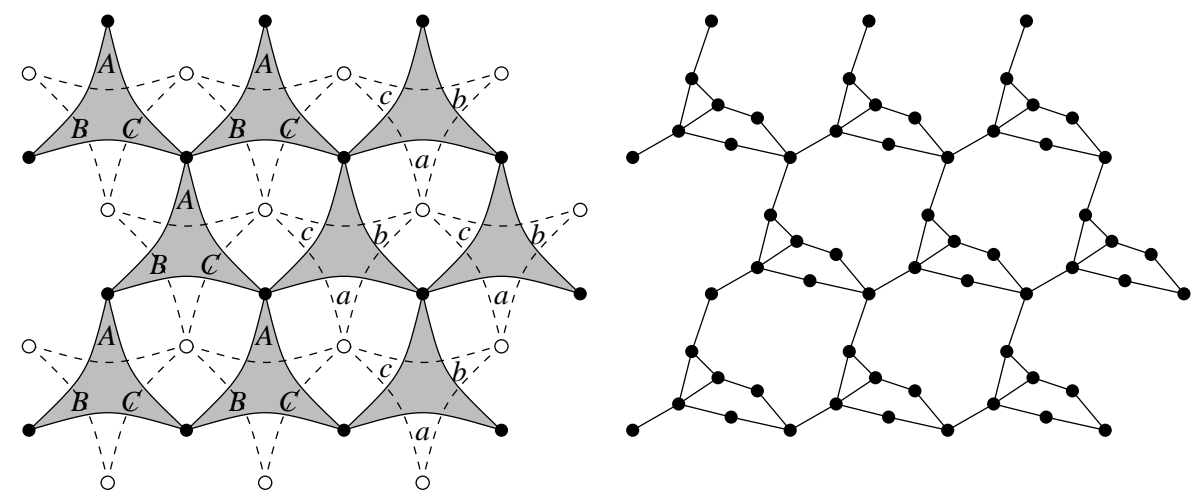

Figure 2.5: The shaded triangles depict the labelled triangular hyperlattice $T$, i.e., the labelled 3-uniform hyperlattice obtained by taking alternate triangles in the triangular lattice, and labelling them consistently; capital letters denote the labelling of $T$. The dashed lines and lower case letters depict the dual $T^{*}$. Note that $T$ is self-dual: there is a rotation through $\pi$ mapping $T$ to $T^{*}$. The figure on the right shows a lattice $L$ obtained by substituting a 'generator' into each hyperedge of $T$.

duals of these partitions are, respectively, $a b c, a b, a c, b c$ and $\emptyset$. In this setting, a probability vector $\mathbf{p}$ is simply a vector $\mathbf{p}=\left(p_{\emptyset}, p_{A B}, p_{A C}, p_{B C}, p_{A B C}\right)$ of nonnegative reals summing to 1 , and the dual of $\mathcal{H}(\mathbf{p})$ is simply $\mathcal{H}^{*}\left(\mathbf{p}^{*}\right)$, where $\mathbf{p}^{*}$ is formed from $\mathbf{p}$ by interchanging $p_{\emptyset}$ and $p_{A B C}$.

The observation of Scullard and Ziff mentioned earlier may be formulated as follows: if the labelled plane hyperlattice $\mathcal{H}$ is self-dual, then the model $\mathcal{H}(\mathbf{p})$ is self-dual if and only if $p_{\emptyset}=p_{A B C}$. The key point is that the other three partitions are all self-dual, as long as the dual triangle is labelled in the appropriate way, so only two entries in the probability vector, namely $p_{\emptyset}$ and $p_{A B C}$, change when we pass to the dual. [In the papers [35, 36], the formulation of duality is not totally clear. Wierman and Ziff [33] clearly formulate the notion

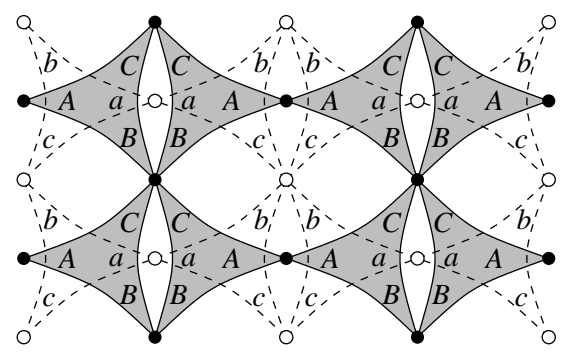

Figure 2.6: A labelled 3-uniform hyperlattice with only translational symmetries that is isomorphic to its dual by reflection in a horizontal line. For visual clarity, the labelling of the dual is indicated with lower case letters. 
of self-duality for unlabelled plane hyperlattices, and state that one can consider any labelling with lattice structure, but this seems to be an oversight; one needs the labelled hyperlattice to be self-dual as a labelled hyperlattice, which is not always the case.]

If the probabilities in $\mathbf{p}$ are appropriate functions of a single parameter $p$, the condition $p_{\emptyset}=p_{A B C}$ allows one to determine the critical point of the model; in general it gives the critical surface. As remarked earlier, Scullard and Ziff do not discuss whether self-duality in fact implies criticality; that it does is shown by Theorem 2.1

Corollary 2.3. Let $\mathcal{H}$ be a labelled 3-uniform plane hyperlattice that is isomorphic (as a labelled plane hyperlattice) to its dual, and let $\mathbf{p}$ be a probability vector $\left(p_{\emptyset}, p_{A B}, p_{A C}, p_{B C}, p_{A B C}\right)$. Then $\mathcal{H}(\mathbf{p})$ percolates if $p_{A B C}>p_{\emptyset}$, and exhibits exponential decay if $p_{A B C}<p_{\emptyset}$.

Proof. Let $\mathbf{q}$ be the probability vector $\left(p, p_{A B}, p_{A C}, p_{B C}, p\right)$, where $p=\left(p_{\emptyset}+\right.$ $\left.p_{A B C}\right) / 2$, and note that $\mathbf{q}^{*}=\mathbf{q}$. Then the dual of the hyperlattice percolation model $\mathcal{H}(\mathbf{q})$ is $\mathcal{H}^{*}\left(\mathbf{q}^{*}\right)=\mathcal{H}^{*}(\mathbf{q})$, which is isomorphic to $\mathcal{H}(\mathbf{q})$ by the assumption on $\mathcal{H}$. Thus $\mathcal{H}(\mathbf{q})$ is a self-dual hyperlattice percolation model, and so is critical by Theorem 2.1. If $p_{A B C}>p_{\emptyset}$, then $\mathbf{p} \succ \mathbf{q}$, while if $p_{A B C}<p_{\emptyset}$, then $\mathbf{p} \prec \mathbf{q}$, so the result follows from Theorem 2.1 .

When $p_{A B C}=p_{\emptyset}$, the model $\mathcal{H}(\mathbf{p})$ may or may not percolate. A (degenerate) example that percolates is given by taking $p_{A B}=1$ and all other probabilities zero in the triangular hyperlattice shown in Figure 2.5. An example that does not is given by taking connection probabilities in the same hyperlattice corresponding to critical bond percolation on the triangular lattice. As we shall see in Section 8, in non-degenerate models there is no percolation at the self-dual point.

As we have seen, Corollary 2.3 follows from Theorem 2.1 simply by restricting the parametrization of the percolation model, using the same partition probabilities for all triangles, rather than allowing different ones for each orbit under the action of the lattice of symmetries. In other words, we took all triangles to be of the same type. Of course, one can restrict the model in other ways, considering two or more types of triangle, or one type of triangle and one type of 4-gon, etc. Since any results obtained in this way are simply special cases of Theorem 2.1 we omit the details; the case of a single type of triangle is of special importance, since (for self-dual $\mathcal{H}$ ), self-duality reduces to a single equation, so one obtains the entire critical surface, rather than a lower dimensional subset of it.

For example, consider the self-dual plane hyperlattice $\mathcal{H}$ shown in Figure 2.7 Depending on the parameters we choose, $\mathcal{H}(\mathbf{p})$ can be self-dual via several different maps $S$ from the plane to itself. Letting $e_{1}$ denote the hyperedge $A B C D$ and $e_{2} x y z w$, there is a translation mapping $e_{1}$ into $e_{2}^{*}$ and $e_{2}^{*}$ into a hyperedge congruent to $e_{1}$. The model $\mathcal{H}(\mathbf{p})$ is self-dual under this translation if and only if the following equations hold: $p_{1, \emptyset}=p_{2, x y z w}, p_{1, A B}=p_{2, x y z}, p_{1, A B C}=p_{2, y z}$, $p_{1, A C}=p_{2, x w \mid y z}, p_{1, A B \mid C D}=p_{2, x z}$, and $p_{1, A B C D}=p_{2, \emptyset}$, together with the 

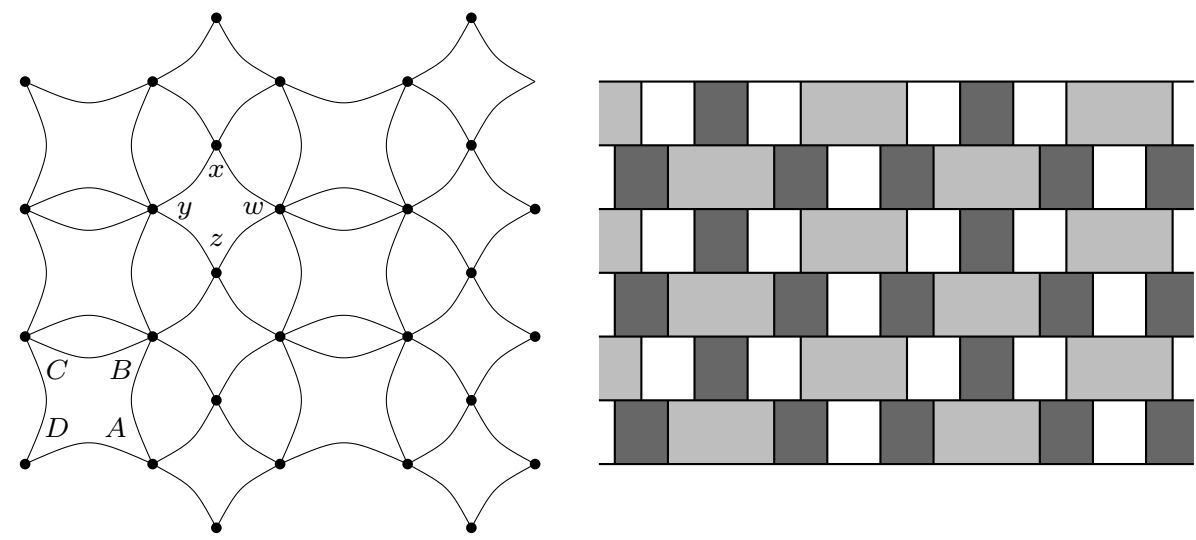

Figure 2.7: Part of a self-dual 4-uniform hyperlattice $\mathcal{H}$, shown both as a plane hypergraph and as the corresponding 3-coloured cubic map.

images of these equations under rotation. One natural way to satisfy these equations is simply to take the same probability $p=1 / 14$ for each of the 14 non-crossing partitions in each square; taking probability $1 / 14$ for all partitions other than that into a single part or into four singletons, and probabilities $p$ and $1 / 7-p$ for these two partitions, it follows that the percolation threshold in this model is at $p=1 / 14$.

Before turning to the proof of Theorem 2.1, let us make some remarks. Firstly, an important special class of hyperlattice percolation models consists of those corresponding to bond or site percolation on (planar) lattices. By a $k$-generator we mean a finite graph $G$ with $k$ distinguished vertices; when $k=3$, we denote these vertices by $A, B$ and $C$. In a planar generator we insist that $G$ is planar, and that the distinguished vertices lie in a common face, which we may take to be the outer face. Suppose that each bond (edge) of $G$ is assigned a probability. Then, taking the bonds open independently with these probabilities, for each partition $\pi$ of the distinguished vertices, let $p_{\pi}$ be the probability that precisely those vertices in the same part of $\pi$ are connected by open paths in $G$. Replacing each hyperedge in a labelled $k$-uniform plane hyperlattice $\mathcal{H}$ by a copy of $G$ one obtains a (planar, if $G$ is planar) lattice $L$. Taking all bonds open independently with the appropriate probabilities, the resulting bond percolation model is equivalent to $\mathcal{H}(\mathbf{p})$ in an obvious sense. In this case we say that $\mathcal{H}(\mathbf{p})$ is bond realizable. We may define site realizability analogously; this time, in each generator we insist that the distinguished vertices are always open. The definitions extend to general plane hyperlattices using one generator for each equivalence class of hyperedges.

The key observation of Ziff [35] and Ziff and Scullard [36] is that, for any selfdual labelled 3-uniform plane hyperlattice $\mathcal{H}$ and any generator $G$, no matter how complicated, the equation $\mathbf{p}=\mathbf{p}^{*}$ reduces to a single polynomial equation in the probabilities associated to the bonds or sites of $G$, so, assuming self- 
duality implies criticality, this equation gives the entire critical surface in the inhomogeneous case. Moreover, if we take the same probability for each bond or site, this polynomial equation gives the critical probability for bond or site percolation on the lattice generated. Indeed, this was how Scullard 24 found the critical point for the 'martini lattice'.

As an example, this method predicts the critical point for bond percolation on the lattice $L$ shown on the right in Figure 2.5, as the root of a certain polynomial of degree 9 . Corollary 2.3 proves that this predicted value is indeed critical. To see the power of the method, consider the original proof of Wierman 31] that the critical probability for bond percolation on the triangular lattice is the $\operatorname{root} 2 \sin (\pi / 18)$ of the equation $p^{3}-3 p+1=0$. This proof relied on the star-triangle transformation, and an apparent coincidence between the various connection probabilities associated to a star and to a triangle. Scullard and Ziff's method gives an argument (which becomes a proof using the results of Wierman and Ziff 33 or Corollary 2.3) that does not rely on this - one simply considers a triangle and writes down the equation that the probability $p^{3}+3 p^{2}(1-p)$ that all vertices are connected is equal to the probability $(1-p)^{3}$ that none are. The fact that one need not consider the dual lattice is key for examples such as the lattice shown on the right in Figure 2.5. which is not simply related to its dual.

We should emphasize that having observed that a certain percolation model is self-dual, one is very far from proving criticality. The classical example is bond percolation on the square lattice with $p=1 / 2$. This model is obviously self-dual; proving the conjectured criticality was one of the key open problems in the early days of percolation theory, finally settled after 20 years by Kesten [16. Since then, criticality at the self-dual point has been proved for a number of other models, but these remain the exceptions. In the hyperlattice context, Wierman and Ziff [33] proved a result of this type using existing results on planar lattices; for this reason they consider only a subclass of bond-realizable models, with planar generators and certain symmetries. This does not include examples such as that in Figure 2.5, which has no symmetries other than translations. However, as outlined earlier, one can easily adapt their method to such lattices, as long as the generator is planar. Chayes and Lei [10] proved a result of this class for triangular hyperlattices; they considered only the symmetric case, and imposed an additional condition on the parameters to ensure positive correlation (see below). They sketched an argument using standard techniques for independent percolation on planar lattices that they claimed extends to this case.

The real significance of Theorem 2.1 is that it applies to models that are not bond or site realizable. In such cases, standard results (such as Menshikov's Theorem [21, for example) do not apply, and there seems to be no simple way to adapt the arguments of Chayes and Lei or Wierman and Ziff. Indeed, considerable work will be needed to prove lemmas corresponding to, for example, Harris's Lemma and the Russo-Seymour-Welsh Lemma. Let us note that there are hyperlattice percolation models that are not bond or site realizable. Indeed, considering a single generator $G$ and the corresponding probabilities $\mathbf{p}$, the event that two given vertices are connected in $G$ is an upset in terms of the states of 
the individual bonds or sites. Thus Harris's Lemma implies that if the vector p is realizable, then for any two upsets $\mathcal{U}_{1}, \mathcal{U}_{2}$ in $\mathcal{P}$ we have $\mathbb{P}\left(\mathcal{U}_{1} \cap \mathcal{U}_{2}\right) \geq$ $\mathbb{P}\left(\mathcal{U}_{1}\right) \mathbb{P}\left(\mathcal{U}_{2}\right)$. For example, we must have $p_{A B C} \geq\left(p_{A B C}+p_{A B}\right)\left(p_{A B C}+p_{B C}\right)$. (In the 3-uniform case, Chayes and Lei [10] show that a necessary and sufficient condition for such positive correlation is that $p_{A B C} p_{\emptyset} \geq p_{A B}\left(p_{B C}+p_{A C}\right)$, and the equations obtained from this by permuting $A, B$ and $C$, all hold.) Of course it is trivial to find a probability vector $\mathbf{p}$ for which this does not hold; an example is given in the introduction.

The applicability of Corollary 2.3 is also not limited to models that are bond or site realizable. Indeed Scullard and Ziff [24, 35, 36] noted that their duality observation does not require the model to be bond or site realizable. As in the original paper of Scullard 24, one may think of any model $\mathcal{H}(\mathbf{p})$ as bond percolation on a suitable (planar) lattice, where the states of the bonds within a generator may be dependent, although those in different generators must be independent. For example, we may take each 3 -generator to be a triangle, and declare that with probability $p_{i, \emptyset}$ none of the edges are open, with probability $p_{i, A B C}$ all three are, with probability $p_{i, A B}$ the edge $A B$ is open and the other edges are closed, and so on. Alternatively, we can take the generator to be a star. However, there is no need to think of bonds at all; for percolation, the only relevant property of the configuration within a triangle is which of the vertices $A, B$ and $C$ the configuration connects to which others, so it is natural to take this (random) partition of the vertices as the fundamental object of study. For the mathematical work (deducing criticality from self-duality), the details of the hyperlattice turn out to be mostly irrelevant; this is why we consider general plane hyperlattices in the rest of the paper.

Finally, let us note that extensions of the Scullard-Ziff criterion to the random cluster model and Potts model have been described by Chayes and Lei [10] and $\mathrm{Wu}$ [34; establishing criticality at the self-dual point remains an open problem in these cases. Returning to hyperlattice percolation, in a few very special cases results have been proved that go further than determining the critical point. For example, Sedlock and Wierman [25] established the equality of the critical exponents between certain pairs of models, and Chayes and Lei [1] extended Smirnov's conformal invariance result 28 , to what is essentially a very restricted case of the present model. In this paper we shall not consider such extensions; rather we shall prove that self-duality does imply criticality, in the full generality of hyperlattice percolation.

\section{A generalization of Harris's Lemma}

Given posets $\mathcal{P}_{1}, \ldots, \mathcal{P}_{n}$, their product is the poset $\mathcal{P}_{1} \times \cdots \times \mathcal{P}_{n}$ where each element $\mathbf{x}$ is a list $\left(x_{1}, \ldots, x_{n}\right)$ with $x_{i}$ an element of $\mathcal{P}_{i}$, with $\mathbf{x} \preccurlyeq \mathbf{y}$ if and only if $x_{i} \preccurlyeq y_{i}$ for $i=1, \ldots, n$. If $\mathbb{P}$ is a product probability measure on a product of posets, then with a slight abuse of notation we write $\mathbb{P}$ for any of the corresponding marginal measures.

In the later sections of this paper we shall make repeated use of the following 
generalization of Harris's Lemma [14 to products of posets. We only need the case where all $\mathcal{P}_{i}$ are equal and finite, but since the proof gives a little more, we state the result more generally. As usual, a greatest element in a poset $\mathcal{P}$ means an element $y$ such that $x \preccurlyeq y$ for all $x \in \mathcal{P}$. Of course, if a greatest element exists, then it is unique.

Lemma 3.1. Let $p>0$. There is a constant $C=C(p)>0$ such that if $\mathbb{P}$ is a product measure on a product $\mathcal{P}=\mathcal{P}_{1} \times \cdots \times \mathcal{P}_{n}$ in which each factor $\mathcal{P}_{i}$ is a poset with a greatest element whose probability is at least $p$, then for any two upsets $A$ and $B$ in $\mathcal{P}$ we have

$$
\mathbb{P}(A \cap B) \geq(\mathbb{P}(A) \mathbb{P}(B))^{C} .
$$

Proof. We shall prove the result with $C=\lceil 2 / p\rceil$.

As usual, we use induction on $n$. When $n=0$, the set $\mathcal{P}$ contains only a single element, and the inequality is trivial. (It is also not hard to verify directly for $n=1$.) Suppose then that $n \geq 1$, and that the result holds for smaller $n$. Suppose for notational convenience that $\mathcal{P}_{n}$ is finite, and list its elements as $x_{0}, x_{1}, \ldots, x_{k}$, with $x_{0}$ the greatest element. Let $p_{i}$ denote the probability of element $i$ in $\mathcal{P}_{n}$, so our assumption is that $p_{0} \geq p$.

Given a set $S \subset \mathcal{P}$, let

$$
S_{i}=\left\{y \in \mathcal{P}_{1} \times \cdots \times \mathcal{P}_{n-1}:\left(y, x_{i}\right) \in S\right\} \subset \mathcal{P}_{1} \times \cdots \times \mathcal{P}_{n-1}
$$

denote the $i$ th slice of $S$ (with respect to the last factor in the product). Clearly, if $S$ is an upset, then so is $S_{i}$, so the induction hypothesis gives $\mathbb{P}\left((A \cap B)_{i}\right) \geq$ $\left(\mathbb{P}\left(A_{i}\right) \mathbb{P}\left(B_{i}\right)\right)^{C}$ for each $i$. Also, the upset conditions give $A_{i} \subset A_{0}$ and $B_{i} \subset B_{0}$ for $i>0$. Since $\mathbb{P}$ is a product measure, we have $\mathbb{P}(S)=\sum_{i} p_{i} \mathbb{P}\left(S_{i}\right)$ for any $S$. Using these observations, it suffices to show that

$$
\sum_{i=0}^{k} p_{i}\left(a_{i} b_{i}\right)^{C} \geq(a b)^{C}
$$

holds whenever the non-negative real numbers $p_{i}, a_{i}$ and $b_{i}$ satisfy the following conditions: $p_{0}, \ldots, p_{k}$ sum to $1, p_{0} \geq p, a_{0}=\max _{i} a_{i}, b_{0}=\max _{i} b_{i}, a=\sum p_{i} a_{i}$, and $b=\sum p_{i} b_{i}$.

In proving (11) we may assume that $a, b>0$. Dividing the $a_{i}$ through by $a$ and the $b_{i}$ by $b$, we may assume that $a=b=1$. Let $\alpha_{i}=a_{i}-1$ and $\beta_{i}=b_{i}-1$. Since $\sum_{i} p_{i} \alpha_{i}=0$, we have $\alpha_{0}=\max _{i} \alpha_{i} \geq 0$. Also,

$$
\sum_{i: \alpha_{i}<0}-p_{i} \alpha_{i}=\sum_{i: \alpha_{i}>0} p_{i} \alpha_{i} \leq \sum_{i} p_{i} \alpha_{0}=\alpha_{0},
$$

and similarly for the $\beta_{i}$. Our aim is to prove that $\sum_{i} p_{i}\left(1+\alpha_{i}\right)^{C}\left(1+\beta_{i}\right)^{C} \geq 1$. Recalling that $\sum_{i} p_{i}\left(\alpha_{i}+\beta_{i}\right)=0$, this is equivalent to showing that

$$
\Delta=\sum_{i} p_{i}\left(\left(1+\alpha_{i}\right)^{C}\left(1+\beta_{i}\right)^{C}-1-C\left(\alpha_{i}+\beta_{i}\right)\right) \geq 0 .
$$


Since $\alpha_{0}, \beta_{0} \geq 0$, we have $\left(1+\alpha_{0}\right)^{C}\left(1+\beta_{0}\right)^{C} \geq 1+C \alpha_{0}+C \beta_{0}+C^{2} \alpha_{0} \beta_{0}$, so the contribution to the sum $\Delta$ from the $i=0$ term is at least $p_{0} C^{2} \alpha_{0} \beta_{0}$.

Turning to the remaining terms, since $(1+x)^{n} \geq 1+n x$ if $x \geq-1$ and $n$ is a positive integer, we have

$$
\left(1+\alpha_{i}\right)^{C}\left(1+\beta_{i}\right)^{C}=\left(1+\alpha_{i}+\beta_{i}+\alpha_{i} \beta_{i}\right)^{C} \geq 1+C\left(\alpha_{i}+\beta_{i}+\alpha_{i} \beta_{i}\right) .
$$

If $\alpha_{i}$ and $\beta_{i}$ have the same sign, then the contribution of the $i$ th summand to (31) is nonnegative. If $\alpha_{i}<0$ and $\beta_{i}>0$, then the negative of the contribution of the $i$ th summand to (3) is at most $p_{i} C\left|\alpha_{i}\right| \beta_{i} \leq p_{i} C\left|\alpha_{i}\right| \beta_{0}$. By (21), the negative of the sum of the contribution of all such terms is at most

$$
C \beta_{0} \sum_{i: \alpha_{i}<0} p_{i}\left(-\alpha_{i}\right) \leq C \beta_{0} \alpha_{0} .
$$

The same bound holds for terms with $\alpha_{i}>0$ and $\beta_{i}<0$, so we conclude that the sum in (3) satisfies

$$
\Delta \geq p_{0} C^{2} \alpha_{0} \beta_{0}-2 C \alpha_{0} \beta_{0}=\left(p_{0} C-2\right) C \alpha_{0} \beta_{0} .
$$

Since $p_{0} C \geq 2$ by our choice of $C$, this establishes the inequality, and hence the lemma.

Note that we have not attempted to optimize the value of $C$ above. Indeed, for $p_{0}$ small, the proof above goes through with $C$ only slightly larger than $1 / p_{0}$, noting that the contribution to $\Delta$ from $i=0$ is at least $C(C-1)\left(\alpha_{0}^{2}+\beta_{0}^{2}\right) / 2+$ $C^{2} \alpha_{0} \beta_{0} \geq\left(C(C-1)+C^{2}\right) \alpha_{0} \beta_{0}$.

Of course, Harris's Lemma itself does not apply in this setting, i.e., one cannot simply take $C=1$. Indeed, considering the upsets $\left\{x_{0}, x_{1}\right\}$ and $\left\{x_{0}, x_{2}\right\}$ in the poset on $\left\{x_{0}, x_{1}, x_{2}\right\}$ in which $x_{0}$ is greatest and $x_{1}$ and $x_{2}$ are incomparable, with $\mathbb{P}\left(x_{0}\right)=p_{0}$ and $\mathbb{P}\left(x_{1}\right)=\mathbb{P}\left(x_{2}\right)=\left(1-p_{0}\right) / 2$, we may have $\mathbb{P}(A)=\mathbb{P}(B)=\left(1+p_{0}\right) / 2$ and $\mathbb{P}(A \cap B)=p_{0}$. For $p_{0}$ small, this shows that we need the exponent $C$ to be at least a constant times $\log \left(1 / p_{0}\right)$.

Since the form of the bound obtained will be irrelevant in our remaining arguments, let us state as a corollary a weaker, more abstract version of the result.

Lemma 3.2. Let $\mathcal{P}$ be a finite poset with a greatest element $x_{0}$ and let $\mathbb{P}$ be a probability measure on $\mathcal{P}$ with $\mathbb{P}\left(x_{0}\right)>0$. There is a function $F=F_{\mathcal{P}, \mathbb{P}}$ from $(0,1]^{2}$ to $(0,1]$ that is strictly increasing in each argument such that, for any $n \geq 1$ and any upsets $A$ and $B$ in $\mathcal{P}^{n}$ with $\mathbb{P}(A), \mathbb{P}(B)>0$, we have

$$
\mathbb{P}(A \cap B) \geq F(\mathbb{P}(A), \mathbb{P}(B)) .
$$

Proof. Immediate from Lemma 3.1.

As usual, the extension to infinite products is immediate by approximating with the finite case. 
Note that while the form of the function $F$ is irrelevant, it is natural to look for an $F$ of the form $F(a, b)=(a b)^{C}$. Indeed, given upsets $A_{i}$ and $B_{i}$ in $\mathcal{P}^{n_{i}}$ then, considering the product upsets $A_{1} \times A_{2}$ and $B_{1} \times B_{2}$ in $\mathcal{P}^{n_{1}+n_{2}}$, one sees that the optimal $F$ satisfies $F\left(a_{1} a_{2}, b_{1} b_{2}\right) \leq F\left(a_{1}, b_{1}\right) F\left(a_{2}, b_{2}\right)$. Of course the optimal $F$ cannot be precisely $F(a, b)=(a b)^{C}$, since we certainly need $C>1$, and then the bound is not tight if $a=1$, for example.

\subsection{High probability unions of upsets}

In many applications of Harris's Lemma in percolation, the exact form of the bound is not important, so the weaker conclusion of the more generally applicable Lemma 3.2 may be used instead of Harris's bound. We give one example that we shall use later: a form of the 'square-root' trick, showing that if the union of a fixed number of upsets has high enough probability, then one of the upsets has high probability.

Corollary 3.3. Let $\mathcal{P}$ be a finite poset with a least element $x_{0}$ and let $\mathbb{P}$ be a probability measure on $\mathcal{P}$ with $\mathbb{P}\left(x_{0}\right)>0$. Given $\varepsilon>0$ and a positive integer $k$ there is a $\delta=\delta(\mathcal{P}, \mathbb{P}, k, \varepsilon)>0$ such that, for any $n \geq 1$, if $A_{1}, \ldots, A_{k}$ are upsets in $\mathcal{P}^{n}$ with $\mathbb{P}\left(\bigcup A_{i}\right) \geq 1-\delta$, then $\mathbb{P}\left(A_{i}\right) \geq 1-\varepsilon$ for some $i$.

Note that $x_{0}$ is a least element here, not a greatest one.

Proof. Set $\delta_{1}=\varepsilon$. For $j \geq 2$ let $\delta_{j}=F\left(\delta_{j-1}, \varepsilon\right)$, where $F$ is the function given by Lemma 3.2 applied to the reverse of $\mathcal{P}$, and set $\delta=\delta_{k} / 2$.

If $\mathbb{P}\left(A_{i}\right)<1-\varepsilon$ for each $i$, then the downsets $A_{i}^{\mathrm{c}}$ each have probability at least $\varepsilon$. Viewing these downsets as upsets in the reversed poset, it follows by Lemma 3.2 and induction on $j$ that $\mathbb{P}\left(A_{1}^{\mathrm{c}} \cap \cdots \cap A_{j}^{\mathrm{c}}\right) \geq \delta_{j}$. Thus $\mathbb{P}\left(\bigcup_{i=1}^{k} A_{i}\right) \leq$ $1-\delta_{k}<1-\delta$, a contradiction.

We shall also need a related result, stating that if we have a union of upsets which is extremely likely to hold, then it is very likely that many of the individual upsets hold, as long as we rule out the trivial case that the union is extremely likely because one of the individual upsets is itself extremely likely.

Lemma 3.4. Let $\mathcal{P}$ be a poset with a least element $x_{0}$, and let $\mathbb{P}$ be a probability measure on $\mathcal{P}$ with $\mathbb{P}\left(x_{0}\right)>0$. Given an integer $N>0$ and a real number $\varepsilon>0$, there exists a $\delta=\delta\left(\mathbb{P}\left(x_{0}\right), N, \varepsilon\right)>0$ such that, for any $n$ and any collection $A_{1}, \ldots, A_{m}$ of upsets in $\mathcal{P}^{n}$ with $\mathbb{P}\left(A_{i}\right) \leq 1-\varepsilon$ for all $i$ and $\mathbb{P}\left(\bigcup A_{i}\right) \geq 1-\delta$, the probability that at least $N$ of the events $A_{i}$ hold is at least $1-\varepsilon$.

Proof. Let $F$ be the function appearing in Lemma 3.2 applied to the reverse of the poset $\mathcal{P}$. Set $\varepsilon^{\prime}=F(\varepsilon / N, \varepsilon)$ and $\delta_{1}=\varepsilon / N$. Inductively define $\delta_{k}$ by $\delta_{k}=F\left(\varepsilon^{\prime}, \delta_{k-1}\right)$ for $k \geq 2$.

We claim that, for any $k \geq 1$, if $A_{1}, \ldots, A_{m}$ is any collection of upsets in any power $\mathcal{P}^{n}$ of $\mathcal{P}$ with $\mathbb{P}\left(\bigcup A_{i}\right) \geq 1-\delta_{k}$, then we can find disjoint index sets $I_{1}, I_{2}, \ldots, I_{k}$ such that for each $1 \leq j \leq k$ we have $\mathbb{P}\left(\bigcup_{i \in I_{j}} A_{i}\right) \geq 1-\varepsilon / N$. The result then follows by setting $\delta=\delta_{N}$ and $k=N$ : the claim tells us that with 
probability at least $1-N \varepsilon / N=1-\varepsilon$, for every $j$ at least one of the events $\left\{A_{i}: i \in I_{j}\right\}$ holds, so at least $N$ of the $A_{i}$ hold.

For $k=1$ the claim is trivial, taking $I_{1}=\{1,2, \ldots, m\}$.

Suppose then that $k \geq 2$ and that the claim holds when we replace $k$ by $k-1$. Let $f_{i}$ be the probability that none of $A_{1}, \ldots, A_{i}$ holds. For any $k$ we have $\delta_{k} \leq \delta_{1}=\varepsilon / N$, so $f_{m} \leq \delta_{k} \leq \varepsilon / N$, and

$$
i_{1}=\min \left\{i: f_{i} \leq \varepsilon / N\right\}
$$

is defined. Setting $I_{1}=\left\{1,2, \ldots, i_{1}\right\}$, note that the event $\bigcup_{j \in I_{1}} A_{i}$ has probability at least $1-\varepsilon / N$.

Consider the downsets $\bigcap_{1 \leq i \leq i_{1}-1} A_{i}^{\mathrm{c}}$ and $A_{i_{1}}^{\mathrm{c}}$. Applying Lemma 3.2 to these events, seen as upsets in the reversed poset, we have $f_{i_{1}} \geq F\left(f_{i_{1}-1}, 1-\right.$ $\left.\mathbb{P}\left(A_{i_{1}}\right)\right)$. Since $F$ is increasing, using the definition of $i_{1}$ and our assumption on $\mathbb{P}\left(A_{i}\right)$, it follows that $f_{i_{1}} \geq F(\varepsilon / N, \varepsilon)=\varepsilon^{\prime}$. Let $\mathcal{D}_{1}=\bigcap_{1 \leq i \leq i_{1}} A_{i}^{\mathrm{c}}$ and $\mathcal{D}_{2}=\bigcap_{i_{1}+1 \leq i \leq m} A_{i}^{\text {c }}$. Applying Lemma 3.2 to $\mathcal{D}_{1}$ and $\mathcal{D}_{2}$, we have

$$
\delta_{k} \geq \mathbb{P}\left(\mathcal{D}_{1} \cap \mathcal{D}_{2}\right) \geq F\left(\mathbb{P}\left(\mathcal{D}_{1}\right), \mathbb{P}\left(\mathcal{D}_{2}\right)\right) \geq F\left(f_{i_{1}}, \mathbb{P}\left(\mathcal{D}_{2}\right)\right) .
$$

Since $F$ is strictly increasing, $f_{i_{1}} \geq \varepsilon^{\prime}$, and $\delta_{k}=F\left(\varepsilon^{\prime}, \delta_{k-1}\right)$, it follows that

$$
F\left(\varepsilon^{\prime}, \delta_{k-1}\right)=\delta_{k} \geq F\left(\varepsilon^{\prime}, \mathbb{P}\left(\mathcal{D}_{2}\right)\right),
$$

so $\mathbb{P}\left(\mathcal{D}_{2}\right) \leq \delta_{k-1}$, and the union of $A_{i_{1}+1}, \ldots, A_{m}$ has probability at least 1 $\delta_{k-1}$. Applying the induction hypotheses to this set of events gives us $I_{2}, \ldots, I_{m}$ with the required properties, completing the proof.

\section{Colourings, hypergraphs and crossings}

Our next aim is to prove a form of rectangle-crossing lemma loosely analogous to the Russo-Seymour-Welsh Lemma [22, 26, but applicable in the hyperlattice percolation context. Naturally, this involves considering 'open crossings of rectangles'. As in [3, 5], for example (see also [6]), to make this precise and clean we shall work instead with 'black crossings' in a suitable black/white colouring of the faces of a cubic map (i.e., 3-regular plane graph). We assume throughout that our maps are well-behaved, meaning that the edges are drawn as piecewise-linear curves, every face is bounded, and any bounded subset of the plane contains only finitely many vertices and meets only finitely many edges.

Recall that a plane hyperlattice $\mathcal{H}$ may be thought of as a 3 -coloured cubic map, where the faces are properly coloured black, white and grey, with a lattice $\mathcal{L}$ of translational symmetries; as usual we view $\mathcal{L}$ as a subset of $\mathbb{R}^{2}$. From now on this is our default viewpoint when considering any plane hyperlattice. A colouring $\mathcal{C}$ of $\mathcal{H}$ is a 2 -coloured cubic map obtained as follows: first subdivide each grey face of $\mathcal{H}$ into one or more subfaces, in such a way that the resulting map is still cubic. Then colour each subface black or white, as in Figure 4.1 for example. The resulting colouring $\mathcal{C}$ is an (improper, of course) black/white colouring of the faces of a (well-behaved) cubic map. It will be convenient to 
$A$

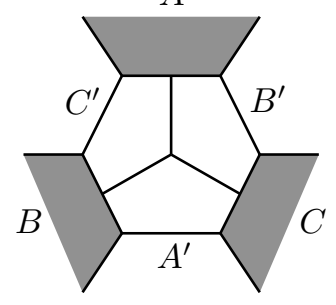

$A$

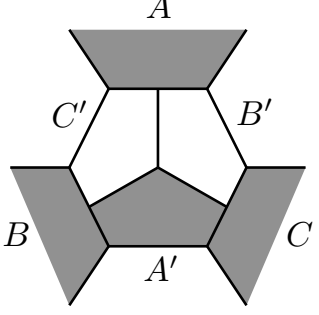

$A$

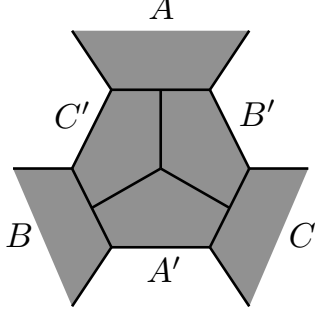

Figure 4.1: Colourings of a grey face (the central hexagon) corresponding to three of the five possible partitions of the vertices (black faces) $A, B, C$ of the corresponding hyperedge, namely the partitions $A|B| C, A \mid B C$ and $A B C$. The outer black faces corresponding to two vertices are connected by a black path inside the hexagon if and only if the vertices are in the same part of the partition; the outer white faces and the white connections between them correspond to the dual partition.

declare that points in the boundary of a face have the colour of that face, so some points are both black and white. A black path in $\mathcal{C}$ is then simply a (piecewise-linear) path in the plane every point of which is black; such a path corresponds to a sequence of black faces in which consecutive faces share a point and thus (since the map is cubic) an edge. White paths are defined similarly.

Recall that a grey face $F_{e}$ of $\mathcal{H}$ corresponds to a hyperedge $e$. Also, since $\mathcal{H}$ is properly 3 -coloured, $F_{e}$ is surrounded alternately by black and white faces, corresponding to vertices and dual vertices. In $\mathcal{C}$, certain pairs of vertices incident with $e$ are connected by black paths within $F_{e}$; this generates a partition $\pi$ of the vertices of $e$, which is easily seen to be non-crossing. Thus $\mathcal{C}$ corresponds to a configuration $\omega$ on $\mathcal{H}$, with open paths in $\omega$ corresponding to black paths in $\mathcal{C}$ and vice versa. (Of course, each open path is represented by many 'nearby' black paths). Crucially, white paths within $F_{e^{*}}=F_{e}$ induce the dual partition $\pi^{*}$ of the dual vertices incident with $e^{*}$, so the negative of $\mathcal{C}$, obtained by interchanging black and white throughout, corresponds to the dual configuration $\omega^{*}$ on $\mathcal{H}^{*}$.

By a black cluster in $\mathcal{C}$ we mean a maximal connected black subset of the plane. A white cycle is a white path that starts and ends at the same point. Since $\mathcal{C}$ is a black/white colouring of a cubic planar map, it is easy to see that a black cluster is surrounded by a white cycle if and only if it is finite. This is a precise form of the duality property relating $\omega$ and $\omega^{*}$ mentioned in Section 2

Remark 4.1. It will be convenient later to assume that all partitions corresponding to $e$ are realized by colourings of a single subdivision of $F_{e}$ into subfaces, as in Figure 4.1. This can be achieved for all non-crossing partitions of hyperedges with any number of vertices, as illustrated in Figure 4.2 .

Note that there are many possible colourings $\mathcal{C}$ corresponding to a given configuration $\omega$ on $\mathcal{H}$ : even if we fix the division of each grey face into subfaces 


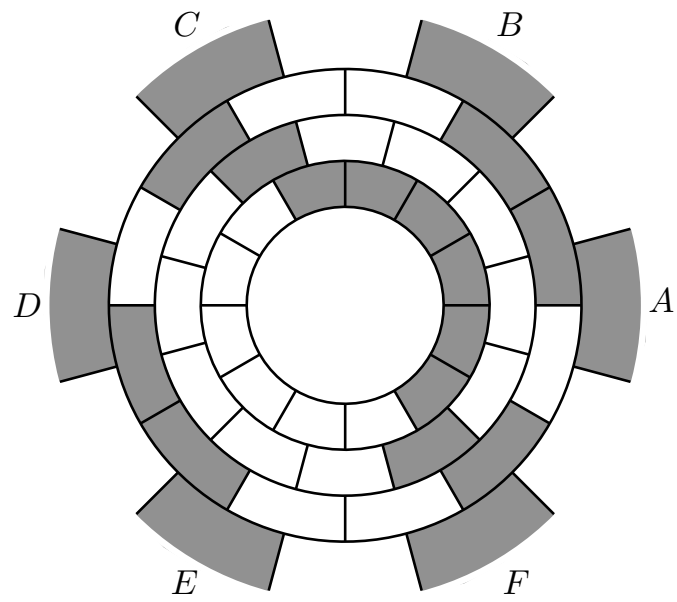

Figure 4.2: A subdivision of the $2 n$-gon corresponding to a hyperedge $e$ with $n=6$ vertices, and a colouring corresponding to the partition $A B|C F| D E$. In general, it suffices to take $2 k-1$ rings of subfaces of the type shown to realize any non-crossing partition, where $k \leq n / 2$ is the maximum 'nesting depth' of a non-crossing partition of $n$ objects.

(as we shall), there may be many colourings of the subfaces giving the same partition of the vertices. For much of the rest of the paper, we shall take the random colouring $\mathcal{C}$ as the fundamental object of study, rather than the random configuration $\omega$.

More formally, an independent lattice colouring $\mathcal{C}$, or simply a colouring, is a colouring obtained from a plane hyperlattice $(\mathcal{H}, \mathcal{L})$ by subdividing each grey face in a deterministic manner, and then colouring the resulting subfaces randomly black and white, in such a way that the colourings inside different grey faces are independent, and translations through elements of the lattice $\mathcal{L}$ preserve the distribution of $\mathcal{C}$. From the remarks above, any plane lattice percolation model $\mathcal{H}(\mathbf{p})$ can be realized by an independent lattice colouring $\mathcal{C}$ associated to $\mathcal{H}$.

We assume throughout that our colourings $\mathcal{C}$ are non-degenerate, meaning that within any grey face, the all-black and all-white colourings have positive probability. Later on we shall have to impose some additional conditions for faces corresponding to hyperedges with more than three vertices.

Note that the state space $\Omega$ underlying the random colouring $\mathcal{C}$ may be viewed as a product of one poset $\mathcal{P}_{F}$ for each grey face $F$ of $\mathcal{H}$ : in the partial order, we have $c_{1} \preccurlyeq c_{2}$ if every subface that is black in $c_{1}$ is black in $c_{2}$. Picking a finite set $F_{1}, \ldots, F_{n}$ of faces representing the orbits of $E(\mathcal{H})$ (the set of grey faces) under the action of $\mathcal{L}$, from lattice invariance we may regard $\Omega$ as a power of the poset $\mathcal{P}=\mathcal{P}_{F_{1}} \times \cdots \times \mathcal{P}_{F_{n}}$. From independence, the probability measure associated to $\mathcal{C}$ is then a product probability measure on $\Omega$. The non-degeneracy 
condition implies that $\mathcal{P}$ has a greatest element (all subfaces of each $F_{i}$ black) and a least element (all white), and that each has positive probability.

The event that a given path is black, or that a black path exists with certain properties, is an upset in $\Omega$, in the sense of Section 3 . Thus Lemma 3.2 applies to two such events. Similarly, considering the reverse poset, Lemma 3.2 applies to two events each defined by the existence of a white path with certain properties. This is the reason for the non-degeneracy assumption.

Note that we are always considering two coloured maps: $\mathcal{H}$ (which is deterministic) and $\mathcal{C}$. To avoid ambiguity, we say that a point or face is $\mathcal{H}$-black if it is black in $\mathcal{H}$, and $\mathcal{C}$-black if it is black in $\mathcal{C}$, and similarly for other colours. By default, black or white refers to $\mathcal{C}$, while grey necessarily refers to $\mathcal{H}$.

The lattice structure ensures that the faces of the hyperlattice $\mathcal{H}$ (seen as a map, as usual) cannot be too wild.

Lemma 4.2. Let $(\mathcal{H}, \mathcal{L})$ be a plane hyperlattice, viewed as a cubic map. There are finitely many faces $F_{1}, \ldots, F_{N}$ of $\mathcal{H}$ such that for any face $F$, there is an element of $\mathcal{L}$ such that the corresponding translation of $\mathbb{R}^{2}$ induces an isomorphism of $\mathcal{H}$ mapping $F$ to one of $F_{1}, \ldots, F_{N}$. Furthermore, there is a constant $d_{0}$ such that (i) every face has diameter at most $d_{0}$ and (ii) every point of $\mathbb{R}^{2}$ is within distance $d_{0}$ of an element of $\mathcal{L}$, and for each $r>0$ there is a constant $N_{r}$ such that any disk of radius $r$ meets at most $N_{r}$ faces.

Proof. Let $D$ be a fundamental domain of $\mathcal{L}$. Since $D$ is bounded, by the definition of a plane hypergraph $D$ contains finitely many vertices of $\mathcal{H}$, and meets finitely many hyperedges. It follows that $D$ meets only finitely many faces of $\mathcal{H}$, viewed now as a cubic map. Hence there is a finite set $F_{1}, \ldots, F_{N}$ of faces, all meeting $D$, containing one representative of each orbit of the action of $\mathcal{L}$ on the faces of $\mathcal{H}$. The remaining statements follow easily, taking $d_{0}$ to be the larger of $\max _{i} \operatorname{diam}\left(F_{i}\right)$ and $\operatorname{diam}(D)$.

The parameter $d_{0}=d_{0}(\mathcal{H})$ appearing in Lemma 4.2 will be used throughout this and the next section. For example, we say that a rectangle is large if all its sides have length at least $100 d_{0}$. In what follows, to avoid trivialities such as a rectangle having a black crossing with probability 1 , we only ever consider large rectangles.

Remark 4.3. Let us remark briefly on the numerical constants appearing in this paper. In many places, rather than argue that some constants exist with certain properties, we simply give numerical values that work, such as 100 (here) or the less natural constants $0.1,1.1,8$, etc appearing later. Of course the precise values are not important.

Given an angle $\theta$, by a $\theta$-aligned rectangle we mean a rectangle $R \subset \mathbb{R}^{2}$ such that one pair of sides makes an angle $\theta$ to the $x$-axis, measured in the positive sense from the $x$-axis. We refer to these sides as horizontal and the other sides as vertical. Thus, after rotating $R$ clockwise through an angle $\theta$, the horizontal sides become horizontal in the usual sense. Whenever we speak of a rectangle 
$R$, we have an angle $\theta$ in mind and assume that $R$ is $\theta$-aligned. Note that the same geometric rectangle is $\theta$-aligned for two values of $\theta$ differing by $\pi / 2$.

By the width and height of a rectangle, we mean the length of the horizontal and vertical sides, respectively; which is which depends on whether we view $R$ as $\theta$-aligned or $(\theta+\pi / 2)$-aligned.

We always assume that our rectangles $R$ are in general position with respect to our colouring $\mathcal{C}$, meaning that each vertex of $R$ lies in the interior of a face of the colouring, no vertices of the colouring are on the boundary of $R$, and the edges of the colouring can only cross the edges of $R$ transversely.

Given a rectangle $R$, by a black horizontal crossing of $R$ we mean a $\mathcal{C}$-black path within $R$ starting at some point on one vertical side of $R$ and ending at some point on the other vertical side. White vertical crossings are defined similarly, and so on. We write $H_{\mathrm{b}}(R)$ for the event that $R$ has a black horizontal crossing (in the random colouring $\mathcal{C}$ ), and $V_{\mathrm{w}}(R)$ for the event that it has a white vertical crossing, and so on. For a proof of the following 'obvious' lemma concerning (well-behaved) 2-coloured maps see [6, Ch. 8, Lemma 12].

Lemma 4.4. Given any well-behaved colouring $\mathcal{C}$ of the plane and any rectangle $R$ in general position with respect to $\mathcal{C}$, exactly one of the events $H_{\mathrm{b}}(R)$ and $V_{\mathrm{w}}(R)$ holds.

This lemma, together with self-duality, will be the starting point for our Russo-Seymour-Welsh-type argument. This argument will be rather involved. There are various technical complications arising from the generality of plane hyperlattices; we deal with most of these in the rest of this section. In the next section we turn to the core of the argument, where the complications are mostly due to the lack of symmetry.

For the rest of the section we consider a given non-degenerate independent lattice colouring $\mathcal{C}$, associated to a plane hyperlattice $\mathcal{H}$.

\subsection{How crossing probabilities vary}

Let $h(R)=\mathbb{P}\left(H_{\mathrm{b}}(R)\right)$ be the probability that $R$ has a black horizontal crossing, and let $v(R)=\mathbb{P}\left(V_{\mathrm{b}}(R)\right)$. Note that if we switch from viewing a given geometric rectangle $R$ as $\theta$-aligned to viewing it as $(\theta+\pi / 2)$-aligned, then $h(R)$ and $v(R)$ swap. Our next aim is to show that $h(R)$ and $v(R)$ do not change too much if we move the edges of $R$ slightly. This is not very surprising, but giving full details in the present generality requires a little work. We start with a technical lemma.

We say that a path $P$ in the plane is potentially black with respect to a hyperlattice $\mathcal{H}$ if no point of $P$ is $\mathcal{H}$-white, so $P$ corresponds to a sequence of black and grey faces of $\mathcal{H}$. In other words, $P$ is potentially black if and only if there is a positive probability that $P$ is actually black in the random colouring $\mathcal{C}$. We write $d_{\mathrm{H}}$ for the Hausdorff distance between subsets of $\mathbb{R}^{2}$. Let $d_{0}=d_{0}(\mathcal{H})$ be the constant given by Lemma 4.2, so every face of $\mathcal{H}$ has diameter at most $d_{0}$. 
Lemma 4.5. Let $\mathcal{H}$ be a hyperlattice and $P$ a piecewise-linear path. Then there is a potentially black path $P^{\prime}$ with $d_{\mathrm{H}}\left(P, P^{\prime}\right) \leq 2 d_{0}$.

Proof. Recall that in the 3 -coloured map $\mathcal{H}$, no two white faces are adjacent. Whenever $P$ passes through a white face, simply take a detour around (or just outside) this face. Similarly, if $P$ starts or ends in a white face, modify $P$ to start/end just outside this face.

Lemma 4.6. Let $\mathcal{C}$ be a non-degenerate independent lattice colouring. Then there exists a constant $c>0$, depending only on (the distribution of) $\mathcal{C}$, such that, for any large rectangle $R$, if $R_{+}$is a rectangle formed by moving one vertical side of $R$ outwards by a distance of at most 1 , then $h(R) \geq h\left(R_{+}\right) \geq c h(R)$.

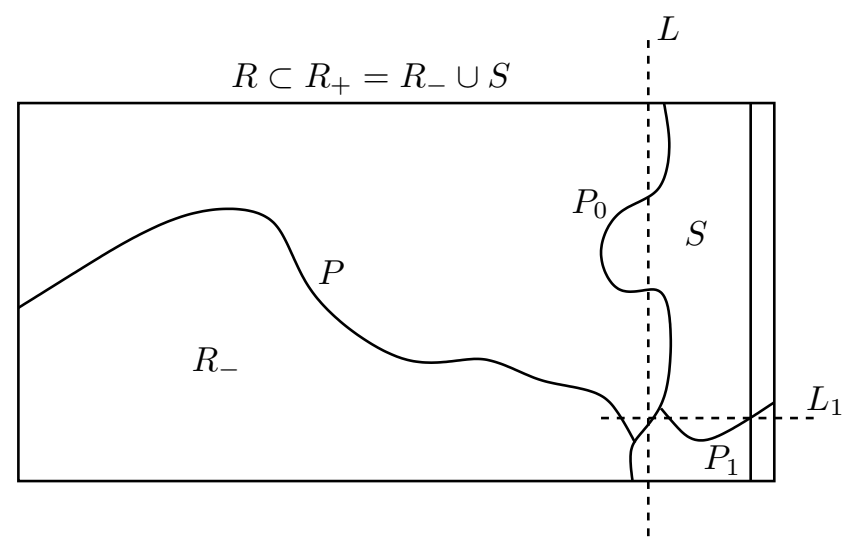

Figure 4.3: A rectangle $R_{+}$slightly extending a rectangle $R$, divided into $R_{-}$ and $S$ by a potentially black path $P_{0}$ that crosses $R$ from top to bottom near its right-hand side. The path $P$ starts on the left-hand side of $R$ and ends on $P_{0}$. Finally, $P_{1}$ starts on $P_{0}$ and ends on the right of $R_{+}$. All paths lie inside $R_{+}$.

Proof. Note first that any black horizontal crossing of $R_{+}$contains a black horizontal crossing of $R$, so $h(R) \geq h\left(R_{+}\right)$. Also, we may assume without loss of generality that we move a side of $R$ outwards by distance exactly 1 to obtain $R_{+}$.

In the arguments that follow, various constants appear that depend on $\mathcal{C}$. However, they will depend only on (a) the quantity $d_{0}=d_{0}(\mathcal{H})$, where $\mathcal{H}$ is the hyperlattice underlying $\mathcal{C}$, and (b) the minimum probability of the all-black state in a grey face. These are invariant under rotation and translation, so, rotating and translating $R$ and $\mathcal{C}$, without loss of generality we may assume that $\theta=0$, so horizontal means horizontal in the usual sense, and that $R=[0, a] \times[0, b]$ and $R_{+}=[0, a+1] \times[0, b]$, even though $h(R)$ itself varies as $R$ is rotated and/or translated with $\mathcal{C}$ fixed. 
We may assume that $a, b \geq 100 d_{0}$. Applying Lemma 4.5 to the line segment $L$ from $\left(a-3 d_{0},-3 d_{0}\right)$ to $\left(a-3 d_{0}, b+3 d_{0}\right)$, and truncating the resulting path $P^{\prime}$ when it last hits the bottom side of $R$ and first hits the top side, we find a potentially black path $P_{0}$ crossing $R$ from top to bottom, where all points have $x$-coordinate between $a-5 d_{0}$ and $a-d_{0}$. Let $S$ be the 'strip' consisting of those points of $R_{+}$to the right of $P_{0}$, and let $R_{-}$denote the rest of $R_{+}$, so $R_{-} \subset R$ is a 'distorted rectangle' whose right-hand side is potentially black. Let $E_{0}$ be the event that $R_{-}$has a horizontal black crossing, noting that if $H_{\mathrm{b}}(R)$ holds then so does $E_{0}$, so $\mathbb{P}\left(E_{0}\right) \geq h(R)$.

Let $\mathcal{F}_{-}$be the set of grey faces meeting $R_{-}$but not $S$, and let $\mathcal{F}_{S}$ be the set of grey faces meeting $S$, so $E_{0}$ depends on the colourings of the faces in $\mathcal{F}_{-} \cup \mathcal{F}_{S}$. Let $E_{0}^{\prime}$ be the event that $E_{0}$ would hold after recolouring (in $\mathcal{C}$ ) all faces in $\mathcal{F}_{S}$ to black; thus $E_{0}^{\prime}$ is the event that there is a path $P$ crossing $R_{-}$ from left to right, every point of which is either $\mathcal{C}$-black or in a face in $\mathcal{F}_{S}$. If $E_{0}$ holds then so does $E_{0}^{\prime}$, so $\mathbb{P}\left(E_{0}^{\prime}\right) \geq \mathbb{P}\left(E_{0}\right) \geq h(R)$.

Now $E_{0}^{\prime}$ depends only on the states of the faces in $\mathcal{F}_{-}$. Let us condition on these states, assuming that $E_{0}^{\prime}$ holds. Our aim is to show that the conditional probability that $H_{\mathrm{b}}\left(R_{+}\right)$holds is not too small. To do this we shall first modify $P$ in a certain way (if needed), and then extend $P$, obtaining a path $P_{+}$crossing $R_{+}$from left to right in which every point is either $\mathcal{C}$-black or in a face in $\mathcal{F}_{S}$, in such a way that the set of faces in $\mathcal{F}_{S}$ that $P_{+}$meets has size $O(1)$. Then we reveal the (as yet unexamined) states of these faces. Since there are $O(1)$ of them, with probability bounded away from zero they are all entirely black in $\mathcal{C}$.

We start with the modification of $P$. Let us call a face bad if it meets both $R_{-}$and $S$, but does not lie entirely in $R_{+}=R_{-} \cup S$, and good otherwise. Since all faces have diameter at most $d_{0}$, any bad face must contain a point within distance $10 d_{0}$ of either $(a, 0)$ or $(a, b)$. In particular, there are $O(1)$ bad faces. Suppose $P$ meets one or more good faces in $\mathcal{F}_{S}$. Tracing $P$ from the left, stop the first time it meets such a face $F$. Since $F$ is connected and meets $S$, we can continue within this face to a point of $S$. Since $F$ is contained in $R_{+}$, in doing so we do not go outside $R_{+}$, but we do leave $R_{-}$, so we cross the right-hand side of $R_{-}$. Stop when this happens.

After this modification, $P$ has the properties above (all points $\mathcal{C}$-black or in faces in $\mathcal{F}_{S}$ ), and it meets at most one good face in $\mathcal{F}_{S}$, and thus (since there are $O(1)$ bad faces in total) $O(1)$ faces in $\mathcal{F}_{S}$.

Let $(x, y)$ be the right-hand end of $P$, so $(x, y)$ lies on $P_{0}$. Pick $y^{\prime} \in\left[3 d_{0}, b-\right.$ $\left.3 d_{0}\right]$ with $\left|y-y^{\prime}\right| \leq 3 d_{0}$. Let $L_{1}$ be the line-segment from $\left(a-7 d_{0}, y^{\prime}\right)$ to $\left(a+1+2 d_{0}, y^{\prime}\right)$. Apply Lemma 4.5 to $L_{1}$ to obtain a potentially black path $P_{1}^{\prime}$. Then $P_{1}^{\prime}$ starts inside $R_{-}$, ends outside $R_{+}$, and cannot cross the lines $y=0$ and $y=b$, so it contains a sub-path $P_{1}$ within $S$ crossing $S$ from left to right. The left-hand end of $P_{1}$ is within distance $10 d_{0}$ of $(x, y)$. To construct our final path $P_{+}$, trace $P$ from left to right, run along $P_{0}$ from $(x, y)$ to the first end of $P_{1}$, and then trace $P_{1}$. This path crosses $R_{+}$from left to right. Furthermore, any point of $P_{+} \backslash P$ is within distance $20 d_{0}$ of $(x, y)$, so $P_{+} \backslash P$ meets $O(1)$ grey faces. Now $P_{+}$is potentially black. Any point of $P_{+}$in a grey face in $\mathcal{F}_{-}$ is necessarily a point of $P$, and so is $\mathcal{C}$-black by the properties of $P$. Finally, 
$P_{+}$meets $O(1)$ grey faces in $\mathcal{F}_{S}$. With (conditional) probability bounded away from 0 the latter faces are all entirely $\mathcal{C}$-black, and then $P_{+}$is a black path, so $H_{\mathrm{b}}\left(R_{+}\right)$holds.

When $h(R)$ is very close to 1 , Lemma 4.6 is not very informative; it does not rule out $h(R)$ dropping from 1 to $1 / 100$, say, as $R$ is extended a tiny bit horizontally. In this case the probability of a white vertical crossing would increase from 0 to $99 / 100$. Note that this vertical crossing probability can change by a large ratio: if the white colouring is subcritical, with a constant and $b$ large, the white vertical crossing probability is approximately of the form $e^{-c_{a} b}$, where $c_{a}$ is a positive constant depending on $a$. Increasing $a$ by 1 decreases $c_{a}$ to a new constant value, which can change $e^{-c_{a} b}$ by an arbitrarily large ratio.

However, it is still true that the white vertical crossing probability cannot jump from very small to fairly large. We phrase the result in terms of black horizontal crossings as above.

Lemma 4.7. Let $\mathcal{C}$ be a non-degenerate independent lattice colouring. Given $\varepsilon>0$ there is a $\delta>0$ such that for any large rectangle $R$ and any rectangle $R_{+}$ formed by moving one vertical side of $R$ outwards by a distance of at most 1 , if $h(R) \geq 1-\delta$ then $h\left(R_{+}\right) \geq 1-\varepsilon$.

Proof. The proof is an extension of that of Lemma 4.6 we define the path $P_{0}$ splitting $R_{+}$into $R_{-}$and $S$ as before, and let $\mathcal{F}_{-}$be the set of grey faces meeting $R_{-}$but not $S$, and $\mathcal{F}_{S}$ the set of grey faces meeting $S$. As before, let $E_{0}^{\prime}$ be the event that $R_{-}$has a horizontal crossing every point of which is $\mathcal{C}$-black or in a grey face in $\mathcal{F}_{S}$, recalling that $\mathbb{P}\left(E_{0}^{\prime}\right) \geq h(R)$.

Let $f_{1}, \ldots, f_{N}$ list all grey or $\mathcal{H}$-black faces of $\mathcal{H}$ meeting $S$. Let $E_{i}$ be the event that $R_{-}$contains a $\mathcal{C}$-black path $P$ starting on the left-hand side of $R_{-}$, ending at a boundary point of $f_{i}$, and meeting no other $f_{j}$, nor the interior of $f_{i}$. Note that $E_{i}$ depends only on the states of grey faces in $\mathcal{F}_{-}$, not those in $\mathcal{F}_{S}$. If $E_{0}^{\prime}$ holds then, truncating a path $P$ witnessing this event the first time $P$ meets any $f_{i}$, we see that one of the $E_{i}$ must hold. Hence,

$$
\mathbb{P}\left(\bigcup E_{i}\right) \geq \mathbb{P}\left(E_{0}^{\prime}\right) \geq h(R)
$$

We claim that any given face $f$ of $\mathcal{H}$ is surrounded by a 'ring' of white and grey faces of $\mathcal{H}$ with bounded size such that if all grey faces in the ring happen to be coloured white in $\mathcal{C}$, then no $\mathcal{C}$-black path starting outside the ring can end at a point of $f$. Here the bound depends only on $\mathcal{H}$, not the face chosen. Indeed, if $f$ is $\mathcal{H}$-black, we simply take the faces neighbouring $f$ to form the ring. If $f$ is white or grey, we may simply take all white or grey faces of $\mathcal{H}$ within distance $2 d_{0}$ of $f$ as our ring: since no black face of $\mathcal{H}$ touches any other black face, any $\mathcal{C}$-black path to $f$ from distance more than $2 d_{0}$ must meet a grey face in our ring.

If every grey face in the ring just described about $f_{i}$ happens to be coloured entirely white in $\mathcal{C}$, then no black path ends at any point of $f_{i}$, and $E_{i}$ does not hold. It follows that for some constant $\varepsilon_{1}>0$ we have $\mathbb{P}\left(E_{i}\right) \leq 1-\varepsilon_{1}$ for all $i$. 
Let $N_{0}$ be the maximum number of faces meeting any disk of radius $100 d_{0}$, and let $N_{1}$ be the number of bad grey or $\mathcal{H}$-black faces, i.e., grey or black faces meeting $S, R_{-}$, and the exterior of $R_{+}$. Note that $N_{0}$ and $N_{1}$ are bounded by constants as before. Let $M$ be a huge constant to be chosen in a moment.

By Lemma 3.4 (applied with $\min \left\{\varepsilon / 2, \varepsilon_{1}\right\}$ in place of $\varepsilon$ ), our assumption $h(R) \geq 1-\delta$, and (44), if we choose $\delta$ small enough, then with probability at least $1-\varepsilon / 2$ at least $K=N_{1}+M N_{0}$ of the events $E_{1}, \ldots, E_{N}$ hold. Let us condition on the states of all faces in $\mathcal{F}_{-}$, assuming that at least $K$ of the $E_{i}$ hold. It suffices to show that the conditional probability that $H_{\mathrm{b}}\left(R_{+}\right)$holds is then at least $1-\varepsilon / 2$. As before, we use the fact that we have not yet looked at the faces in $\mathcal{F}_{S}$.

Since there are at most $N_{1}$ bad faces among the $f_{i}$, there is a set $I_{0}$ of size at least $M N_{0}$ such that for every $i \in I_{0}$ the event $E_{i}$ holds and $f_{i}$ is good. Using the greedy algorithm, we may pick a subset $I \subset I_{0}$ of size at least $M$ such that for distinct $i, j \in I_{0}$ the faces $f_{i}$ and $f_{j}$ are at distance at least $30 d_{0}$. For $i \in I_{0}$, let $P_{i}$ be a path witnessing $E_{i}$. Our aim is to complete the proof as before, but now showing that each $P_{i}$ has a not-too-small chance of being extendable to cross $R_{+}$, and that these events (that the particular extensions we look for are present) are independent, so with high probability at least one hold. The details are essentially as before: since $f_{i}$ is good, we may extend $P_{i}$ within the face $f_{i}$ to meet our right-hand side $P_{0}$. Then we find an extension $P_{i}^{+}$of $P_{i}$ as before, remaining within distance $10 d_{0}$ of the end of $P_{i}$. The extensions meet disjoint sets of faces, so we are done.

Together, Lemmas 4.6, 4.7 and 4.4 show that no crossing probability changes 'too much' when a rectangle is moved slightly. This statement needs a little interpretation: we could in principle obtain explicit bounds in Lemmas 4.6 and 4.7. However, these turn out to be irrelevant. In the end, all we care about is whether certain probabilities tend to 0 or tend to 1 as some parameter (the area of the rectangles we consider) tends to infinity. It will thus be convenient to 're-scale' all probabilities by an increasing function $\varphi:(0,1) \rightarrow \mathbb{R}$ with $\varphi(x) \rightarrow-\infty$ as $x \rightarrow 0$ and $\varphi(x) \rightarrow \infty$ as $x \rightarrow 1$, in such a way that the maximum change in a probability $p$ 'allowed' by our lemmas corresponds to a change in $\varphi(p)$ of at most 2 , say.

To make this precise, let $c_{\mathrm{b}}$ be the constant given by Lemma 4.6 recall that this does not depend on the orientation of $R$. Let $c_{\mathrm{w}}$ be the corresponding constant with black and white exchanged, and let $c_{0}=\min \left\{c_{\mathrm{b}}, c_{\mathrm{w}}\right\}$. Similarly, given $\varepsilon>0$, let $\delta_{0}(\varepsilon)=\min \left\{\delta_{\mathrm{b}}, \delta_{\mathrm{w}}\right\}$, where $\delta_{\mathrm{b}}=\delta_{\mathrm{b}}(\varepsilon)$ is given by Lemma 4.7 and $\delta_{\mathrm{w}}$ by Lemma 4.7 with black and white exchanged.

Define a sequence $\left(\varepsilon_{n}\right)_{n>0}$ inductively by setting $\varepsilon_{0}=1 / 2$ and $\varepsilon_{n+1}=$ $\min \left\{c_{0} \varepsilon_{n}, \delta_{0}\left(\varepsilon_{n}\right)\right\}$. Set $\pi_{n}=1-\varepsilon_{n}$ for $n \geq 0$ and $\pi_{n}=\varepsilon_{-n}$ for $n \leq 0$. Consider the scaling function $\varphi:(0,1) \rightarrow \mathbb{R}$ defined as follows: set $\varphi\left(\pi_{n}\right)=n$ for all $n \in$ $\mathbb{Z}$, and interpolate linearly between these points. Note that $\varphi(1-p)=-\varphi(p)$. This function (or rather its inverse) is illustrated in Figure 4.4.

Recall that we call a rectangle large if both sides have length at least $100 d_{0}$. 


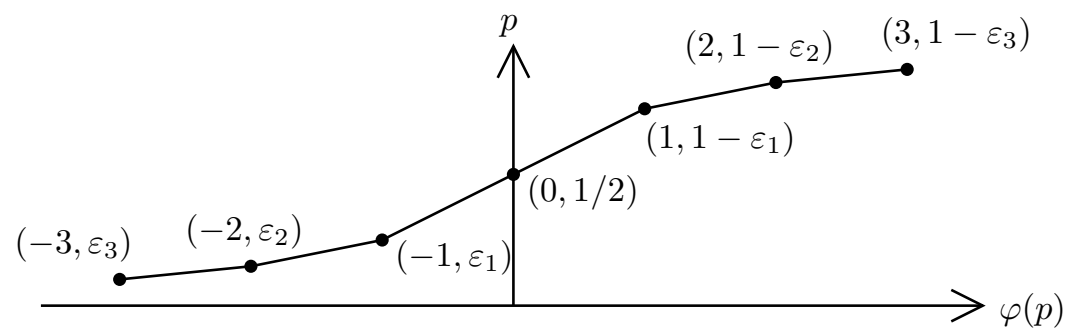

Figure 4.4: The inverse of the scaling function $p \mapsto \varphi(p)$.

Lemma 4.8. Let $R_{1}$ and $R_{2}$ be two large rectangles such that one of $R_{1}$ and $R_{2}$ is obtained from the other by moving one side outwards by a distance between 0 and 1 . Let $f(R)$ be any of the four functions $\mathbb{P}\left(H_{\mathrm{b}}(R)\right), \mathbb{P}\left(H_{\mathrm{w}}(R)\right), \mathbb{P}\left(V_{\mathrm{b}}(R)\right)$ and $\mathbb{P}\left(V_{\mathrm{w}}(R)\right)$. Then $\left|\varphi\left(f\left(R_{1}\right)\right)-\varphi\left(f\left(R_{2}\right)\right)\right| \leq 2$.

Proof. Without loss of generality, $R_{2}$ extends $R_{1}$ horizontally. Using Lemma 4.4 and the fact that $\left|\varphi\left(1-p_{1}\right)-\varphi\left(1-p_{2}\right)\right|=\left|-\varphi\left(p_{1}\right)+\varphi\left(p_{2}\right)\right|=\left|\varphi\left(p_{1}\right)-\varphi\left(p_{2}\right)\right|$, we may assume that we are considering horizontal crossings. Exchanging colours, we may assume that $f(R)=\mathbb{P}\left(H_{\mathrm{b}}(R)\right)=h(R)$. Let $p_{j}=f\left(R_{j}\right)=h\left(R_{j}\right)$, noting that $p_{1} \geq p_{2}$. Let $i=\left\lfloor\varphi\left(p_{1}\right)\right\rfloor$, so $\pi_{i} \leq p_{1}<\pi_{i+1}$. If $i \leq 0$ then by definition of $\pi_{i}$ we have $\pi_{i-1}=\varepsilon_{-i+1} \leq c_{0} \varepsilon_{-i}=c_{0} \pi_{i}$. By Lemma 4.6 we have $p_{2} \geq c_{0} p_{1}$, so $p_{2} \geq \pi_{i-1}$ and $\varphi\left(p_{2}\right) \geq i-1$.

On the other hand, if $i \geq 1$ then $\pi_{i}=1-\varepsilon_{i} \geq 1-\delta_{0}\left(\varepsilon_{i-1}\right)$. Since $p_{1} \geq \pi_{i}$, Lemma4.7 gives $p_{2} \geq 1-\varepsilon_{i-1}=\pi_{i-1}$. In either case we have $\pi_{i-1} \leq p_{2} \leq p_{1}<$ $\pi_{i+1}$, so $i-1 \leq \varphi\left(p_{2}\right) \leq \varphi\left(p_{1}\right)<i+1$ and the result follows.

Lemma 4.8 shows that if we measure probabilities in the right way, they don't change too much when we move a rectangle slightly. Since we have a lattice of translational symmetries, this has the following consequence. (Recall that a rectangle is 'large' if its sides have length at least $100 d_{0}$, where $d_{0}=d_{0}(\mathcal{H})$ is the constant from Lemma 4.2.)

Corollary 4.9. There is a constant $C$ such that if $R^{\prime}$ is a translate of a large rectangle $R$, then $\left|\varphi(h(R))-\varphi\left(h\left(R^{\prime}\right)\right)\right| \leq C$.

Proof. Pick a fundamental domain $D$ of the lattice $\mathcal{L}$ of symmetries. Since our colouring $\mathcal{C}$ is invariant under translations corresponding to elements of $\mathcal{L}$, we may assume that $R$ and $R^{\prime}$ are related by translation by a vector in $D$. Since $D$ is bounded, the result follows by applying Lemma 4.8 a bounded number of times.

Define $\psi:(0,1) \rightarrow(0,1)$ by $\psi(p)=\varphi^{-1}(\varphi(p)-C)$, where $C$ is the constant given by Corollary 4.9. Then $\psi$ is increasing. In fact, although we shall not use this, $\psi$ is strictly increasing, and $\psi(p)$ tends to 0 as $p \rightarrow 0$ and to 1 as $p \rightarrow 1$. We may rewrite Corollary 4.9 is the following more convenient form. 
Corollary 4.10. Suppose that $R$ and $R^{\prime}$ are large rectangles with the same orientation, width and height. Then $h\left(R^{\prime}\right) \geq \psi(h(R))$ and $v\left(R^{\prime}\right) \geq \psi(v(R))$.

We may also rotate a rectangle slightly without changing the crossing probabilities much.

Corollary 4.11. Let $R$ be any rectangle of width $a$ and height $b$, with $a, b \geq$ $100 d_{0}$ (so $R$ is large), and let $R^{\prime}$ be obtained from $R$ by rotating it about its centre through an angle $\theta \leq 1 /(10 \max \{a, b\})$. If $f(\cdot)$ denotes any of the four crossing probability functions considered in Lemma 4.8, then $\left|\varphi(f(R))-\varphi\left(f\left(R^{\prime}\right)\right)\right| \leq 8$.

Proof. Without loss of generality we may assume that $f$ is the function $f(\cdot)=$ $h(\cdot)$ giving the probability of a black horizontal crossing. Rotating and translating the rectangles and $\mathcal{C}$ together as before (or simply changing coordinates) we may assume that $R=[-a, a] \times[-b, b]$. Let $R^{\prime \prime}=[-a-1, a+1] \times[-b+1, b-1]$. Then any horizontal crossing of $R^{\prime \prime}$ crosses $R^{\prime}$ horizontally, so $h\left(R^{\prime}\right) \geq h\left(R^{\prime \prime}\right)$. Hence, by Lemma $4.8 \varphi\left(h\left(R^{\prime}\right)\right) \geq \varphi\left(h\left(R^{\prime \prime}\right)\right) \geq \varphi(h(R))-8$.

A similar argument interchanging $R$ and $R^{\prime}$ gives $\varphi(h(R)) \geq \varphi\left(h\left(R^{\prime}\right)\right)-8$, so the result follows.

A key consequence of the lemma above is that for any given orientation, we may find a large rectangle $R$ with $h(R)$ not too close to 0 or 1 , and that when we rotate, we can assume that the dimensions of $R$ vary 'smoothly'. For now we formalize only the first of these statements. Given an angle $\theta$, we write $h_{\theta}(m, n)$ and $v_{\theta}(m, n)$ for $\mathbb{P}\left(H_{\mathrm{b}}(R)\right)$ and $\mathbb{P}\left(V_{\mathrm{b}}(R)\right)$, where $R$ is an $m$-by- $n \theta$ aligned rectangle centred on the origin. (Thus $v_{\theta}(m, n)=h_{\theta+\pi / 2}(n, m)$ )

Lemma 4.12. Let $\mathcal{C}$ be a non-degenerate independent lattice colouring associated to a hyperlattice $\mathcal{H}$. Given $L \geq 100 d_{0}$, there is a constant $A_{0}$ that for any $A \geq A_{0}$ and any angle $\theta$, there are $m, n \geq L$ with $m n=A$ such that $-4 \leq \varphi\left(h_{\theta}(m, n)\right) \leq 4$.

Proof. By Lemma 4.2 there is a constant $N=N(L)$ such that any disk of radius $2 L$ meets at most $N$ faces of $\mathcal{H}$. By Lemma 4.5. if $R$ is a rectangle of any orientation with width $m \geq L$ and height $n=L$, then we can find $\left\lfloor m /\left(10 d_{0}\right)\right\rfloor \geq m /\left(20 d_{0}\right)$ potentially white paths $P_{i}$ crossing $R$ from top to bottom, with these paths separated by distances of at least $d_{0}$. Since the events that these paths are white are independent, and each path meets at most $N$ grey faces, the probability that no $P_{i}$ is white is at most $\exp (-\alpha m)$ for some constant $\alpha>0$ that does not depend on $\theta$. By Lemma 4.4 if any $P_{i}$ is white, then $H_{\mathrm{b}}(R)$ does not hold. Taking $A$ large enough (i.e., $A \geq L^{2}$ and $A \geq$ $\left.L \alpha^{-1}\left|\log \left(\varphi^{-1}(-4)\right)\right|\right)$, we thus have $\varphi\left(h_{\theta}(A / L, L)\right) \leq-4$; similarly, if $A$ is large enough then $\varphi\left(h_{\theta}(L, A / L)\right) \geq 4$.

Now consider a rectangle $R$ with area $A$ that varies smoothly between these two extremes, centred always on the origin. As $m$ varies, Lemma 4.8 implies that $\varphi\left(h_{\theta}(m, A / m)\right)$ cannot jump by more than 8 at any point, and the result follows. 


\section{A rectangle-crossing lemma}

In the original context of independent bond percolation on the square lattice, there are now several different proofs of the key lemma of Russo 22] and Seymour and Welsh 26; ; see, for example, 4, 5, 3. The various proofs extend (with differing degrees of additional complication) to various more general classes of percolation model. However, as far as we are aware, none of the published proofs can be made to work in the context of general self-dual hyperlattices - in addition to various technical problems, they all require symmetry assumptions that may not hold here.

In this section we shall prove an analogue of the Russo-Seymour-Welsh Lemma for self-dual hyperlattice percolation. Since this proof is a little involved, we first illustrate the key ideas by writing out the argument for bond percolation on $\mathbb{Z}^{2}$. This amounts to reproving the original RSW Lemma in a more complicated way than necessary. Even among proofs using our new strategy, we do not aim to present the simplest, but rather one that extends easily to hyperlattices.

\subsection{Bond percolation on $\mathbb{Z}^{2}$}

Throughout this subsection we consider independent bond percolation on $\mathbb{Z}^{2}$ with $p=1 / 2$. Thus a configuration $\omega$ is an assignment of a state (open or closed) to each edge $e$ of $\mathbb{Z}^{2}$, and $\mathbb{P}$ is the probability measure on the set $\Omega=2^{E\left(\mathbb{Z}^{2}\right)}$ of configurations in which the states $\omega(e)$ of different bonds are independent and each bond is open with probability $1 / 2$.

All rectangles $R$ we consider will be aligned with the axes and have corners with integer coordinates; a rectangle includes its boundary. An open horizontal crossing of $R$ is a path of open bonds in $R$ joining a vertex on the left to one on the right; we write $H(R)$ for the event that $R$ has such a crossing. Similarly, $V(R)$ is the event that $R$ has an open vertical crossing, defined analogously.

As inputs to the argument we shall present, we need two simple lemmas, which do make use of the symmetries of $\mathbb{Z}^{2}$; the main argument will then use only translational symmetries. The first lemma is a standard fact which is an easy consequence of the self-duality of $\mathbb{Z}^{2}$. It is well known to hold with $c_{1}=1 / 2$; see [4, for example. We write $c_{1}$ rather than $1 / 2$ since the main argument below does not depend on the particular value of $c_{1}$, and in the case of hyperlattices, the value of $c_{1}$ in the analogous statement will be different.

Lemma 5.1. There is a constant $c_{1}>0$ such that if $S$ is any square in $\mathbb{Z}^{2}$ then $\mathbb{P}(H(S))=\mathbb{P}(V(S)) \geq c_{1}$.

Our second 'input lemma' is the following consequence of Lemma 5.1 whose proof also requires the use of symmetry. Here we can take $c_{2}=c_{1}^{2} / 16$, but again the value of $c_{2}$ is irrelevant later.

Lemma 5.2. Given an n-by-n square $S$, let $E=E(S)$ be the event that there is an open vertical crossing $P$ of $S$ such that the $x$-coordinates of the endpoints 
of $P$ differ by at most $3 n / 5$. There is a constant $c_{2}>0$ such that $\mathbb{P}(E(S)) \geq c_{2}$ for all squares $S$.

Proof. Let $c_{1}>0$ be as in Lemma 5.1 and consider $S=[0, n]^{2}$. We may suppose that $\mathbb{P}(E)<c_{1} / 2$. Let $F_{1}$ be the event that $S$ has an open vertical crossing from some point $(x, 0)$ to some point $\left(x^{\prime}, n\right)$ with $x^{\prime}-x>3 n / 5$, and $F_{2}$ the horizontal mirror image of this event. Now $V(S)=E \cup F_{1} \cup F_{2}$, so we must have $\mathbb{P}\left(F_{i}\right) \geq c_{1} / 4$ for some $i$. Since $\mathbb{P}\left(F_{1}\right)=\mathbb{P}\left(F_{2}\right)$ by symmetry, it follows that $\mathbb{P}\left(F_{1}\right)=\mathbb{P}\left(F_{2}\right) \geq c_{1} / 4$. But then, by Harris's Lemma, $\mathbb{P}\left(F_{1} \cap F_{2}\right) \geq c_{1}^{2} / 16$. Suppose that $F_{1}$ and $F_{2}$ hold, and let $P_{1}$ and $P_{2}$ be open paths witnessing these

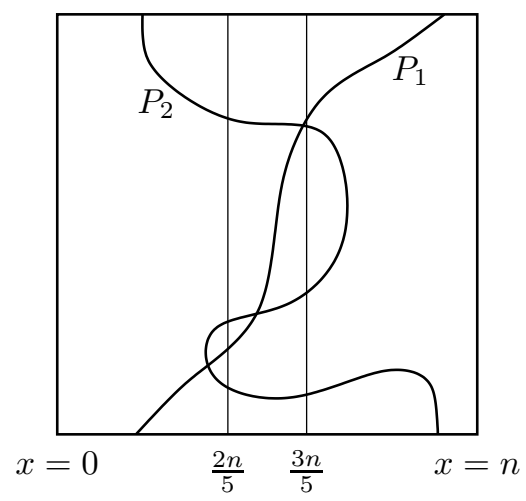

Figure 5.1: Two open paths crossing a square $S$ vertically, $P_{1}$ from bottom-left to top-right, and $P_{2}$ from bottom-right to top-left. Their union contains a path $P$ from bottom-left to top-left.

events, with $P_{i}$ joining $\left(x_{i}, 0\right)$ to $\left(x_{i}^{\prime}, n\right)$; see Figure 5.1 Since $x_{1}^{\prime}-x_{1} \geq 3 n / 5$, we have $x_{1} \leq 2 n / 5$ and $x_{1}^{\prime} \geq 3 n / 5$, and similarly $x_{2} \geq 3 n / 5$ and $x_{2}^{\prime} \leq 2 n / 5$. It follows that $P_{1}$ and $P_{2}$ cross. Hence there is an open path $P$ within $S$ joining $\left(x_{1}, 0\right)$ to $\left(x_{2}^{\prime}, n\right)$. Since $0 \leq x_{1}, x_{2}^{\prime} \leq 2 n / 5$, this shows that $E$ holds. In conclusion, if $\mathbb{P}(E)<c_{1} / 2$, then $\mathbb{P}(E) \geq c_{1}^{2} / 16$, so $\mathbb{P}(E) \geq c_{1}^{2} / 16>0$.

Let us write $h(m, n)=\mathbb{P}(H(R))$ for the probability that a rectangle $R$ of width $m$ and height $n$ has an open horizontal crossing, and $v(m, n)=\mathbb{P}(V(R))$ for the probability that it has an open vertical crossing. Our aim is to prove the following form of the RSW Lemma.

Theorem 5.3. There is a constant $c>0$ such that $v(n, 100 n) \geq c$ for all $n$.

Of course, Theorem 5.3 is a well known result of Russo 22 and Seymour and Welsh [26. As noted above, our aim in this subsection is to present a (complicated) way of deducing Theorem 5.3 from Lemmas 5.1 and 5.2 using minimal properties of the measure $\mathbb{P}$. In particular, we shall restrict ourselves to properties that extend to general hyperlattice percolation, so the argument will adapt to prove Theorem 5.12 below. For example, we shall use translational symmetry but no other symmetry. We shall make repeated use of Harris's 
Lemma, and of the geometric fact that open horizontal and vertical crossings of the same rectangle must meet.

We also use one more very important property of the measure $\mathbb{P}$ : if a rectangle $R$ has an open horizontal crossing, then it has an uppermost open horizontal crossing $U H(R)$ with the property that the event $U H(R)=P_{0}$ is independent of the states of all bonds below $P_{0}$. Indeed, $U H(R)$ may be found by 'exploring $R$ from above'; see [4, for example. Similarly, if $H(R)$ holds then $R$ has a lowest open horizontal crossing $L H(R)$, defined analogously, and found by exploring from below.

The proof of Theorem 5.3 that we shall present, although simpler than that of Theorem 5.12 is still somewhat lengthy. We shall start with three lemmas, the first two of which are standard observations.

In what follows, we shall often implicitly assume that $n$ is 'large enough', meaning larger than a suitable constant $n_{0}$ depending on the parameters, e.g., $\varepsilon$, that we choose. To avoid clutter, we ignore the rounding of plane coordinates to integers; it is easy to see that the effect of rounding can be handled by adjusting the various constants suitably. (Recall that in this subsection we are not proving new results, merely rehearsing the arguments that we shall use in the next subsection; formally, nothing outside this subsection depends on anything inside it. So we do not feel the need to dot all i's and cross all t's.)

Lemma 5.4. For any $\varepsilon>0$ and $c^{\prime}>0$ there is a $c>0$ such that for any $n$, if $v(n,(1+\varepsilon) n)>c^{\prime}$ then $v(n, 100 n)>c$.

Proof. Given $\gamma \geq 1$, let $R_{1}$ and $R_{3}$ be rectangles of width $n$ and heights $\gamma n$ and $(1+\varepsilon) n$, respectively, overlapping in a square $R_{2}$ of side $n$. If $V\left(R_{1}\right), H\left(R_{2}\right)$ and $V\left(R_{3}\right)$ all hold, then so does $V\left(R_{1} \cup R_{3}\right)$; see Figure 5.5 for an illustration of this in a slightly different context. By the original form of Harris's Lemma 14, it follows that

$$
v(n,(\gamma+\varepsilon) n) \geq v(n, \gamma n) h(n, n) v(n,(1+\varepsilon) n) .
$$

Since $v(n, n) \geq c_{1}$ and $h(n, n) \geq c_{1}$ by Lemma [5.1. applying (5) inductively $\lceil 99 / \varepsilon\rceil$ times gives the result.

Given two overlapping $n$-by- $n$ squares $S_{1}$ and $S_{2}$ such that $S_{2}$ is obtained from $S_{1}$ by translating it upwards through a distance of at most $n$, let $T=$ $T\left(S_{1}, S_{2}\right)$ denote the infinite strip bounded by the vertical lines containing the vertical sides of $S_{1}$ and $S_{2}$. Let $J\left(S_{1}, S_{2}\right)$ be the event that $S_{1}$ and $S_{2}$ have open horizontal crossings $P_{1}$ and $P_{2}$ that are joined within $T$, meaning that there is an open path $P$ within $T$ joining some point of $P_{1}$ to some point of $P_{2}$; this includes the case where $P_{1}$ and $P_{2}$ meet; see Figure 5.2 Note that when $J\left(S_{1}, S_{2}\right)$ holds, a minimal $P$ lies entirely between $P_{1}$ and $P_{2}$ in the strip, so we may assume that $P$ is contained in $S_{1} \cup S_{2}$. For later, note also that if there is an open horizontal crossing $P_{1}$ of $S_{1}$ above an open horizontal crossing $P_{2}$ of $S_{2}$, then $P_{1}$ is in fact contained in $S_{2}$, and the crossings $P_{1}$ of $S_{1}$ and $P_{1}$ of $S_{2}$ meet, so $J\left(S_{1}, S_{2}\right)$ holds. 
Lemma 5.5. For any $\varepsilon>0$ and $c^{\prime}>0$ there is a $c>0$ such that for any $n$, if there exist $n$-by-n squares $S_{1}$ and $S_{2}$ with $S_{2}$ obtained by translating $S_{1}$ upwards by a distance of $\varepsilon n$ such that $\mathbb{P}\left(J\left(S_{1}, S_{2}\right)\right) \geq c^{\prime}$, then $v(n, 100 n) \geq c$.
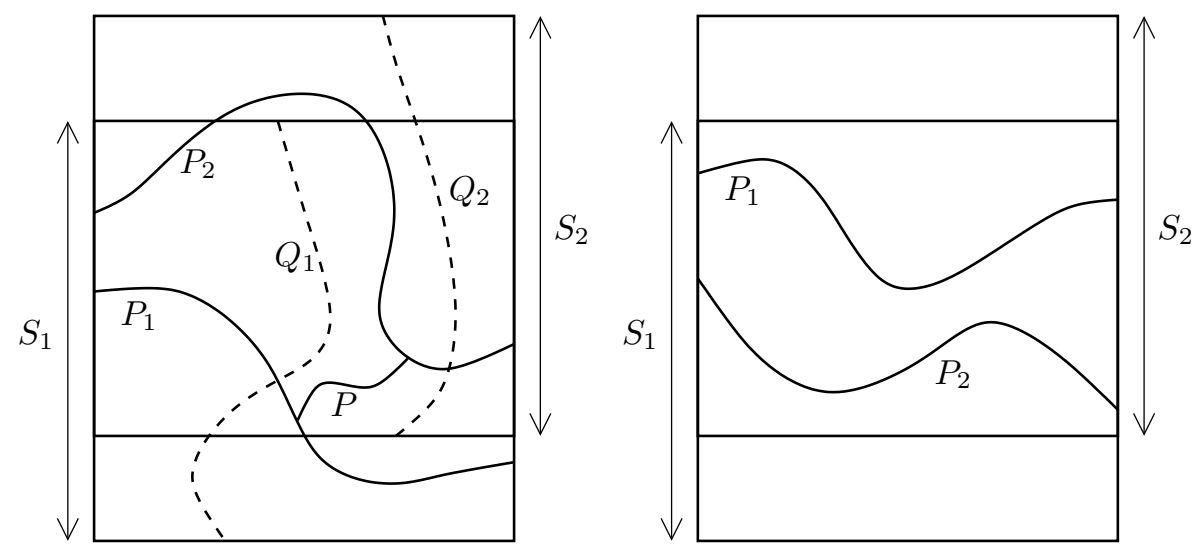

Figure 5.2: In the left figure, the solid paths illustrate the event $J\left(S_{1}, S_{2}\right)$. The dashed paths $Q_{i}$ are vertical crossings of the squares $S_{i}$. Since $P_{i}$ and $Q_{i}$ meet, the event $V\left(S_{1} \cup S_{2}\right)$ holds. The figure on the right shows that if any horizontal crossing of $S_{1}$ is above any horizontal crossing of $S_{2}$, then $J\left(S_{1}, S_{2}\right)$ holds either crossing crosses both squares.

Proof. The event $J=J\left(S_{1}, S_{2}\right)$ is increasing. Hence, by Harris's Lemma and Lemma 5.1, the event $E=J \cap V\left(S_{1}\right) \cap V\left(S_{2}\right)$ has probability at least $c_{1}^{2} c^{\prime}$.

Using the fact that horizontal and vertical crossings of the same square must meet, it is easy to see that whenever $E$ holds, so does $V\left(S_{1} \cup S_{2}\right)$; see Figure 5.2 Hence $v(n,(1+\varepsilon) n)=\mathbb{P}\left(V\left(S_{1} \cup S_{2}\right)\right) \geq \mathbb{P}(E) \geq c_{1}^{2} c^{\prime}$, and the result follows by applying Lemma 5.4 with $c_{1}^{2} c^{\prime}$ in place of $c^{\prime}$.

Our next lemma is less run-of-the-mill. Given $\varepsilon>0$ and two $n$-by- $n$ squares $S_{1}$ and $S_{2}$ with $S_{2}$ obtained by translating $S_{1}$ upwards by a distance of $\varepsilon n / 10$, define the strip $T=T\left(S_{1}, S_{2}\right)$ as above, and let $G_{\varepsilon}\left(S_{1}, S_{2}\right)$ be the event that $H\left(S_{1}\right)$ and $H\left(S_{2}\right)$ hold, the path $P_{1}=L H\left(S_{1}\right)$ is below $P_{2}=U H\left(S_{2}\right)$ in $T$, and the area of $T$ between $P_{1}$ and $P_{2}$ is at most $\varepsilon n^{2}$. In other words, the lowest open horizontal crossing of the lower square and the highest of the higher square do not meet, but they are 'close together', in the sense that the area between them is small.

Lemma 5.6. For any $0<\varepsilon<1 / 10$ there are constants $c_{3}>0$ and $c>0$ such that for any $n$, either there exist $n$-by-n squares $S_{1}$ and $S_{2}$ as above with $\mathbb{P}\left(G_{\varepsilon}\left(S_{1}, S_{2}\right)\right) \geq c_{3}$, or $v(n, 100 n)>c$.

Proof. Set $N=2\lceil 2 / \varepsilon\rceil$, and, for $i=0,1, \ldots, N$, let $S_{i}=[0, n] \times[i \varepsilon n / 10, n+$ $i \varepsilon n / 10]$, so $S_{i+1}$ is obtained by translating $S_{i}$ upwards through a distance $\varepsilon n / 10$. 
Let $H=H\left(S_{0}\right) \cap \ldots \cap H\left(S_{N}\right)$. Since each $H\left(S_{i}\right)$ has probability at least $c_{1}$, by Harris's Lemma there is some $c^{\prime}>0$ such that $\mathbb{P}(H) \geq c^{\prime}$. (We may take $c^{\prime}=c_{1}^{N+1}$, but we prefer to be less specific, with an eye to the hyperlattice case.) Set $c_{3}=c^{\prime} /(2 N)$. If for some $i$ the event $J\left(S_{i}, S_{i+1}\right)$ has probability at least $c_{3}$, then we are done by Lemma 5.5. Let $J=\bigcup_{i=0}^{N-1} J\left(S_{i}, S_{i+1}\right)$. Then we may assume that $\mathbb{P}(J) \leq N c_{3} \leq c^{\prime} / 2$. Hence $\mathbb{P}(H \backslash J) \geq c^{\prime} / 2$.

We claim that if $H \backslash J$ holds, then so does $F=\bigcup_{i=0}^{N-1} G_{\varepsilon}\left(S_{i}, S_{i+1}\right)$. Assuming the claim, the result follows, since for some $i$ we have

$$
\mathbb{P}\left(G_{\varepsilon}\left(S_{i}, S_{i+1}\right)\right) \geq N^{-1} \mathbb{P}(H \backslash J) \geq c^{\prime} /(2 N)=c_{3} .
$$

Suppose then that $H \backslash J$ holds, and let $P_{i}^{-}$and $P_{i}^{+}$be the lowest and highest open horizontal crossings of $S_{i}$. Note that $P_{i}^{-}$and $P_{i}^{+}$may meet, but $P_{i}^{-}$lies (weakly) below $P_{i}^{+}$. Since $J$ does not hold, for $i=0, \ldots, N-1$, the path $P_{i}^{+}$is strictly below $P_{i+1}^{-}$; it follows that $P_{i}^{-}$is strictly below $P_{i+1}^{-}$. For $i=0, \ldots, N-2$, let $A_{i}$ be the region in $T$ between $P_{i}^{-}$and $P_{i+2}^{-}$. Then the $A_{i}$ are disjoint. Since there are $\lfloor N / 2\rfloor \geq 2 / \varepsilon$ such regions $A_{i}$, and their union is contained in a rectangle of width $n$ and height $n+N \varepsilon n / 10 \leq n+(6 / \varepsilon) \varepsilon n / 10<$ $2 n$, it follows that some $A_{i}$ has area at most $\varepsilon n^{2}$. Since the region between $P_{i}^{-}$ and $P_{i+1}^{+}$is contained in $A_{i}$, it follows that $G_{\varepsilon}\left(S_{i}, S_{i+1}\right)$ holds, as required.

Clearly, in the present context the events $G_{\varepsilon}\left(S_{i}, S_{i+1}\right)$ considered above all have the same probability due to translational symmetry; with an eye to the general case, we avoided using this fact.

We now turn to the key idea, which is a rather involved way of generating a configuration. Given a configuration $\omega$ and a vector $v \in \mathbb{Z}^{2}$, let $\omega^{v}$ be obtained by translating $\omega$ through the vector $v$. Thus the state of a bond $e$ in $\omega^{v}$ is the state of its translate $e-v$ in $\omega$. Let us say that a random configuration $\omega$ has the standard distribution if it has the distribution corresponding to $\mathbb{P}$, so bonds are open independently in $\omega$ and each is open with probability $1 / 2$. From translation invariance, if $\omega$ is random with the standard distribution and $v$ is constant, then $\omega^{v}$ has the standard distribution. This conclusion also holds if $v$ is random, as long as $\omega$ and $v$ are independent.

Suppose we have some algorithm $\mathbb{A}$ whose input is a configuration $\omega$, and that $\mathbb{A}$ examines the states of bonds one by one, with the next bond to be examined depending on the results of previous examinations, but not on the states of any other bonds. We assume that $\mathbb{A}$ terminates after a finite number of steps, and write $\mathcal{S}=\mathcal{S}_{\mathbb{A}}(\omega)$ for the set of bonds examined by $\mathbb{A}$ when $\mathbb{A}$ is run on the configuration $\omega$. Let $\omega_{1}$ and $\omega_{2}$ be independent standard configurations. Define a new configuration $\omega$ by running $\mathbb{A}$ on $\omega_{1}$, setting $\omega(e)=\omega_{1}(e)$ if $e \in \mathcal{S}_{\mathbb{A}}\left(\omega_{1}\right)$ and $\omega(e)=\omega_{2}(e)$ otherwise. Then it is easy to check that $\omega$ has the standard distribution: we can think of tossing coins corresponding to $\omega_{1}$ to determine the states of all bonds, looking at certain bonds (corresponding to $\mathcal{S}$ ), and then retossing the coins we have not yet looked at.

Given an algorithm $\mathbb{A}$ as above, and a corresponding map $\mathcal{S}_{\mathbb{A}}$ from the set $\Omega$ of all configurations to the set of subsets of $E\left(\mathbb{Z}^{2}\right)$, define a map $f_{\mathbb{A}}$ from 
$\Omega \times \Omega \times \mathbb{Z}^{2}$ to $\Omega$ by

$$
\left(f_{\mathbb{A}}\left(\omega_{1}, \omega_{2}, v\right)\right)(e)= \begin{cases}\omega_{1}(e) & \text { if } e \in \mathcal{S}_{\mathbb{A}}\left(\omega_{1}\right), \\ \omega_{2}(e-v) & \text { if } e \notin \mathcal{S}_{\mathbb{A}}\left(\omega_{1}\right) .\end{cases}
$$

Combining the two observations above, we see that if $\omega_{1}, \omega_{2}$ and $v$ are independent and $\omega_{1}$ and $\omega_{2}$ have the standard distribution, then $\omega=f_{\mathbb{A}}\left(\omega_{1}, \omega_{2}, v\right)$ does too.

Proof of Theorem 5.3. Recall that our task is to show that the probability $v(n, 100 n)$ that an $n$-by-100n rectangle has an open vertical crossing is bounded away from zero, using Lemmas 5.1 and 5.2 as 'inputs', and otherwise making no use of reflectional or rotational symmetry.

With an eye to later generalizations, suppose that $\alpha, \beta_{1}, \beta_{2}$ and $\eta$ are positive constants satisfying

$$
\alpha, \beta_{1} \leq 1 / 3, \quad \beta_{2} \leq 2, \quad \text { and } \eta>3 \beta_{1} .
$$

Let $E_{\mathrm{v}}=E_{\mathrm{v}}(n)$ be the event that the rectangle $\left[0, \beta_{1} n\right] \times\left[0, \beta_{2} n\right]$ contains an open path from some point $(x, y)$ to some point $\left(x^{\prime}, y^{\prime}\right)$ with $y^{\prime} \geq y+\alpha n$ and $\left|x-x^{\prime}\right| \leq(1-\eta)\left|y-y^{\prime}\right|$. (Here 'v' stands for 'vertical': the overall orientation of the path is significantly closer to vertical than to horizontal.) Taking $\alpha=$ $\beta_{1}=\beta_{2}=1 / 100$ and $\eta=1 / 10$, Lemma 5.2 tells us that for all (large enough) $n$, we have $\mathbb{P}\left(E_{\mathrm{v}}(n)\right) \geq c_{2}>0$. In the rest of the proof we assume only that our various constants satisfy (77) and that, for these constants, $\mathbb{P}\left(E_{\mathrm{v}}\right)$ is bounded away from 0 .

Pick $\gamma>0$ such that

$$
\gamma \leq 1 / 3 \text { and } \eta \geq 3 \beta_{1}+3 \gamma,
$$

and choose $\varepsilon>0$ such that $\varepsilon<\gamma$ and

$$
\varepsilon<\gamma^{2} \alpha / 10
$$

Let $c_{3}$ and $c$ be the constants given by Lemma 5.6. For any $n$, by Lemma 5.6 either $v(n, 100 n) \geq c$, in which case we are done, or there are squares $S_{1}$ and $S_{2}$ with $S_{2}$ obtained by translating $S_{1}$ upwards by a distance of $\varepsilon n / 10$ such that

$$
\mathbb{P}\left(G_{\varepsilon}\left(S_{1}, S_{2}\right)\right) \geq c_{3} .
$$

We may assume that the second case holds. By translational symmetry, we may assume that $S_{1}=[0, n]^{2}$ and $S_{2}=[0, n] \times[\varepsilon n / 10,(1+\varepsilon / 10) n]$.

Recall that $J=J\left(S_{1}, S_{2}\right)$ is the event that there are open horizontal crossings of $S_{1}$ and $S_{2}$ that meet, or are connected by an open path lying within the strip

$$
T=\{(x, y): 0 \leq x \leq n\} \subset \mathbb{R}^{2}
$$

generated by $S_{1} \cup S_{2}$. Also, $G_{\varepsilon}=G_{\varepsilon}\left(S_{1}, S_{2}\right)$ is the event that $H\left(S_{1}\right)$ and $H\left(S_{2}\right)$ hold, the path $P_{1}=L H\left(S_{1}\right)$ is below $P_{2}=U H\left(S_{2}\right)$ in $T$, and the area of $T$ between $P_{1}$ and $P_{2}$ is at most $\varepsilon n^{2}$. We shall show that

$$
\mathbb{P}\left(J \cap G_{\varepsilon}\right) \geq c^{\prime}
$$


for some constant $c^{\prime}$ depending on the various constants we have chosen so far, but not on $n$. Then $\mathbb{P}(J) \geq c^{\prime}$ and so, applying Lemma 5.5 and reducing $c$ if necessary, we have $v(n, 100 n) \geq c$, as required.

Consider the following algorithm $\mathbb{A}$ for testing whether $G_{\varepsilon}$ holds: explore $S_{1}$ from below (as in [4) to find its lowest horizontal crossing $P_{1}$, if $H\left(S_{1}\right)$ holds. Similarly, explore $S_{2}$ from above to find its uppermost horizontal crossing $P_{2}$, if $H\left(S_{2}\right)$ holds; then, from the positions of $P_{1}$ and $P_{2}$, decide whether $G_{\varepsilon}$ holds. Define $f_{\mathbb{A}}$ as in (6) above.

Let $\omega_{1}, \omega_{2}$ and $X$ be independent, where the $\omega_{i}$ are random configurations (with the standard distribution) and $X$ is uniformly random on $[-5 n, 5 n-1]^{2}=$ $\{-5 n,-5 n-1, \ldots, 5 n-1\}^{2} \subset \mathbb{Z}^{2}$, and let $\omega=f_{\mathbb{A}}\left(\omega_{1}, \omega_{2}, X\right)$, so $\omega$ has the standard distribution. The reason for the slightly incongruous notation is that the core of our argument will involve conditioning on $\omega_{1}$ and $\omega_{2}$, but keeping $X$ random.

To establish (11), we first examine $\omega$ to check whether $\omega \in G_{\varepsilon}$. This depends only on the states of bonds examined by the algorithm $\mathbb{A}$ above; by the definition of $f_{\mathbb{A}}$, the state of such bonds in $\omega$ is the same as in $\omega_{1}$. Thus $\omega \in G_{\varepsilon}$ if and only if $\omega_{1} \in G_{\varepsilon}$. When this event holds, let $P_{1}$ and $P_{2}$ be the paths defined above, and write $A$ for the part of the strip $T$ on or below $P_{1}, B$ for the part of $T$ on or above $P_{2}$, and $G$ for the 'gap' between $P_{1}$ and $P_{2}$, i.e., the rest of $T$; see Figure 5.3. Note that all bonds whose interiors lie within $G$ have their states in $\omega=f_{\mathbb{A}}\left(\omega_{1}, \omega_{2}, X\right)$ given by $\omega_{2}^{X}$. Suppose that $\omega_{2}$ contains an open path $P$ from a point $u$ to a point $v$. Then $\omega_{2}^{X}$ contains the open path $P+X$, the translate of $P$ through $X$, joining $u+X$ to $v+X$. If $u+X \in A, v+X \in B$, and $P+X$ remains within the strip $0 \leq x \leq n$, then the minimal subpath $P^{\prime}$ of $P+X$ meeting $A$ and $B$ contains bonds only in $G$, so this path $P^{\prime}$ is present in $\omega$ and joins $P_{1}$ and $P_{2}$, and $\omega \in J$.

Let $E$ be the event that $\omega_{1} \in G_{\varepsilon}$ and $\omega_{2} \in E_{\mathrm{v}}$. Since $\omega_{1}$ and $\omega_{2}$ are independent, we have $\mathbb{P}(E)=\mathbb{P}\left(G_{\varepsilon}\right) \mathbb{P}\left(E_{\mathrm{v}}\right)>c_{3} c_{2}$. For the rest of the proof we condition on $\omega_{1}$ and $\omega_{2}$, so the only remaining randomness is in the choice of $X$. We assume that $E$ holds; we shall show that for any $\omega_{1}$ and $\omega_{2}$ such that $E$ holds, the conditional probability that $J$ holds satisfies

$$
\mathbb{P}\left(J \mid \omega_{1}, \omega_{2}\right) \geq c_{4}=\gamma^{2} \alpha / 400 .
$$

Then we have $\mathbb{P}(J) \geq c_{4} \mathbb{P}(E) \geq c_{4} c_{3} c_{2}>0$, establishing (11). It remains only to prove (12).

Let us choose an open path $P$ in the configuration $\omega_{2}$ witnessing $\omega_{2} \in E_{\mathrm{v}}$; thus $P$ lies within $\left[0, \beta_{1} n\right] \times\left[0, \beta_{2} n\right]$ and joins some point $(x, y)$ to some $\left(x^{\prime}, y^{\prime}\right)$ with $y^{\prime}-y \geq \alpha n$ and $\left|x^{\prime}-x\right| \leq(1-\eta)\left|y-y^{\prime}\right|$. Let $v$ be the vector $\left(x^{\prime}-x, y^{\prime}-y\right)$. Let $I=\left\lceil(1+2 \gamma) n /\left(y^{\prime}-y\right)\right\rceil$, and note that

$$
I \leq\lceil(1+2 \gamma) / \alpha\rceil \leq 2 / \alpha
$$

since $y^{\prime}-y \geq \alpha n$ and $\alpha, \gamma \leq 1 / 3$. For $0 \leq i \leq I$, set $w_{i}=i v$; we think of $w_{i}$ as an offset, for reasons that will hopefully become clear. 
Let $P^{*}$ be the ('virtual', in the sense that it is not known to be open in any configuration we are considering) path formed by starting at the origin and concatenating $I$ copies of $P$. Thus $P^{*}$ is the union of the paths $P_{1}^{*}, \ldots, P_{I}^{*}$, where each $P_{i}^{*}$ is the translate of $P$ joining the point $w_{i-1}$ to $w_{i}$; see Figure 5.3
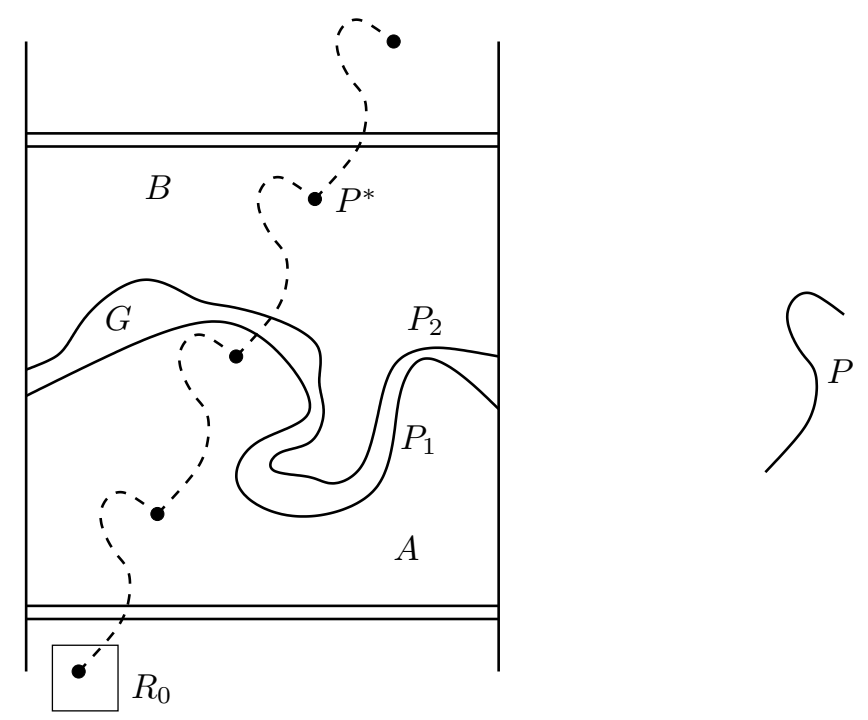

Figure 5.3: The main part of the figure shows the lowest crossing $P_{1}$ of $S_{1}$ and highest crossing $P_{2}$ of $S_{2}$, dividing the strip $T$ into the regions $A, G$ and $B$. In the final configuration $\omega$, bonds in $A \cup B$ have their states given by $\omega_{1}$; those in $G$ are given by a random translate of $\omega_{2}$. On the right, an open path $P$ in $\omega_{2}$ is shown; on the left we see a 'virtual' chain $P^{*}$ of translates of $P$, starting somewhere in the square $R_{0}$. Since $G$ is small, for most starting points in $R_{0}$, at least one copy of $P$ in $P^{*}$ crosses from $A$ to $B$. It follows that with not-toosmall probability, the random translate of $P$ present in $\omega_{2}^{X}$ crosses from $A$ to $B$, giving an open path in $\omega$ joining $P_{1}$ to $P_{2}$.

If $x^{\prime} \geq x$, let $v_{0}$ be the point $\left(\left(\beta_{1}+\gamma / 2\right) n,-\gamma n / 2\right)$; otherwise, set $v_{0}=$ $\left(n-\left(\beta_{1}+\gamma / 2\right) n,-\gamma n / 2\right)$. We claim that if a translate $\widetilde{P}^{*}$ of $P^{*}$ starts at a point within the square $R_{0}$ of side $\gamma n$ centred at $v_{0}$, then this translate lies entirely within the strip $T$. Indeed, in the case $x^{\prime} \geq x$, the $x$-coordinate of any point of $\widetilde{P}^{*}$ is within $\left(\beta_{1}+\gamma / 2\right) n$ of the $x$-coordinate of some point $v_{0}+w_{i}$, and hence is at least 0 and at most

$$
\begin{aligned}
\left(2 \beta_{1}+\gamma\right) n+I\left(x^{\prime}-x\right) & \leq\left(3 \beta_{1}+\gamma\right) n+(I-1)\left(x^{\prime}-x\right) \\
& \leq\left(3 \beta_{1}+\gamma\right) n+(I-1)(1-\eta)\left(y^{\prime}-y\right) \\
& \leq\left(3 \beta_{1}+\gamma+(1-\eta)(1+2 \gamma)\right) n \leq n,
\end{aligned}
$$

using the assumption that $\eta \geq 3 \beta_{1}+3 \gamma$ in the final step. In the case $x^{\prime}<x$ the argument is similar, subtracting all $x$-coordinates from $n$. 
Note that $R_{0}$ lies entirely below $S_{1}$, so, recalling that $A$ and $B$ are the portions of the strip $T$ that lie below $P_{1}$ and above $P_{2}$, respectively, we have $R_{0} \subset A$.

If a translate of $P^{*}$ starts at a point in $R_{0}$, then it ends at a point with $y$-coordinate at least $-\gamma n+I\left(y^{\prime}-y\right) \geq-\gamma n+(1+2 \gamma) n=(1+\gamma) n \geq(1+\varepsilon) n$, so its upper endpoint is above $S_{2}$, and hence lies in $B$.

For any point $v \in R_{0}$, we have seen that $v+w_{0}=v \in A$, while $v+w_{I} \in B$. Also, $v+w_{i} \in T=A \cup G \cup B$ for $0 \leq i \leq I$. Hence, either some point $v+w_{i}$, $1 \leq i<I$, lies in the 'gap' $G$, or there is some $i$ such that $v+w_{i-1} \in A$ and $v+w_{i} \in B$. Let us colour the points of $R_{0}$ with $I+1$ colours, assigning colour 0 in the first case, and colour $i$ in the second (choosing the minimal $i$ if there are several). Let $C_{i}$ denote the set of points in $R_{0}$ assigned colour $i$.

Now $C_{0}$ is a subset of the union of $I-1$ translates of $G$. Since $G$ has area at most $\varepsilon n^{2}$, while $I \leq 2 / \alpha$, the area of $C_{0}$ is thus at most $2 \varepsilon n^{2} / \alpha \leq \gamma^{2} n^{2} / 5$, recalling (91). Since $R_{0}$ has area $\gamma^{2} n^{2}$, it follows that there is some $i>0$ for which $C_{i}$ has area at least $I^{-1} \gamma^{2} n^{2} / 2 \geq \gamma^{2} \alpha n^{2} / 4$.

Recall that in constructing our random configuration $\omega$ as $\omega=f_{\mathbb{A}}\left(\omega_{1}, \omega_{2}, X\right)$, we shift the configuration $\omega_{2}$ by a random vector $X$ uniformly distributed on (the integer points in) $[-5 n, 5 n-1]^{2}$. Recall also that the open path $P$ in $\omega_{2}$ starts at $(x, y) \in\left[0, \beta_{1} n\right] \times\left[0, \beta_{2} n\right] \subset[0,2 n]^{2}$. Consider the set $C_{i}^{\prime}=C_{i}+w_{i-1}-(x, y)$. Then $C_{i}^{\prime}$ has the same area as $C_{i}$, and certainly lies within $[-5 n, 5 n-1]^{2}$. Hence the probability that $X$ falls in $C_{i}^{\prime}$ is at least $\left(\gamma^{2} \alpha n^{2} / 4\right) /\left(100 n^{2}\right)=\gamma^{2} \alpha / 400$. But when this happens, the translate $P+X$ of $P$ starts at a point of $C_{i}+w_{i-1}$. Hence we may think of $P+X$ as the path $P_{i}^{*}=P_{1}^{*}+w_{i-1}$ in a translate of $P^{*}$ starting at a point of $C_{i}$. From the comments above and the definition of $C_{i}$ it follows that $P+X$ lies entirely within $T$, starts in $A$, and ends in $B$. As noted earlier, the presence of such a path in $\omega_{2}^{X}$ guarantees that the configuration $\omega=f_{\mathbb{A}}\left(\omega_{1}, \omega_{2}, X\right)$ has the property $J$. Hence the conditional probability that $\omega$ belongs to $J$ given $\omega_{1}$ and $\omega_{2}$ is at least $\gamma^{2} \alpha / 400$, establishing (12) and completing the proof.

Remark 5.7. The key step of the proof above involved selecting an open path $P$ in $\omega_{2}$, and then chaining together translates of $P$ to form a path $P^{*}$ with the following properties: $P^{*}$ stays well within the strip $T$, starts well below $S_{1}$, and ends well above $S_{2}$, where 'well within' means at least a distance $\gamma n / 2$ away from the boundary. This elbow room ensures that we can translate $P^{*}$ through distances of up to $\gamma n / 2$ while retaining the properties of starting below $S_{1}$, ending above $S_{2}$, and remaining within $T$. Then we randomly shifted the whole path $P^{*}$, and randomly chose one of the links in the chain to focus on, thinking of this link as being the final random shift $P^{\prime}$ of the path $P$ in $\omega_{2}$. Since it is unlikely that any randomly shifted link starts or ends in the gap $G$, there is always at least one link that crosses from $A$ to $B$, so the probability that $P^{\prime}$ does so is bounded away from zero. In order to construct a suitable $P^{*}$, we required that $P$ remain within a fairly small region, and that its endpoints be significantly further apart vertically than horizontally.

It is just as easy to start from more than one path in $\omega_{2}$. Indeed, suppose 
that there are constants $c>0$ and $C$ such that with probability at least $c$ the configuration $\omega_{2}$ contains a set of open paths $P_{j}$ such that we can chain together at most $C$ paths $P_{i}^{*}$, each of which is a translate of some $P_{j}$, to form a path $P^{*}$ with the properties above. Note that this will hold (for example) whenever $\omega_{2}$ contains paths $P_{1}$ and $P_{2}$ each of which stays within some not-too-large region, such that the overall directions of $P_{1}$ and $P_{2}$ are significantly different, and each is not too short, in the sense that the vector from the start to the end is not too short. Then one can always chain $O(1)$ copies together to produce an approximation to a vertical line. Taking $w_{i-1}$ to be the start of the $i$ th path in the chain as above, the proof goes through essentially unaltered, except that at the very end we set $C_{i}^{\prime}=C_{i}+w_{i-1}-\left(x_{j}, y_{j}\right)$ if the $i$ th path in our chain is a copy of $P_{j}$, where $\left(x_{j}, y_{j}\right)$ is the starting point of $P_{j}$.

\subsection{A rectangle-crossing lemma for hyperlattices}

Our aim in this subsection is to prove an analogue of Theorem 5.3 in the context of percolation on plane hyperlattices; we have already illustrated the main ideas in a simpler context in the previous subsection.

Recall that a plane hyperlattice $(\mathcal{H}, \mathcal{L})$, originally defined as an embedding of a hypergraph, may also be defined simply as a cubic map whose faces are properly coloured black, white and grey, in a way that is invariant under translations through elements of the lattice $\mathcal{L}$; see Section 2 , Recall from Section 4 that an independent lattice colouring $\mathcal{C}$ associated to $\mathcal{H}$ is a random black/whitecoloured map obtained from $\mathcal{H}$ as follows: First subdivide each grey face into subfaces in a deterministic manner, keeping the resulting map cubic. Then recolour these subfaces randomly black and white, with the colourings inside different grey faces independent, such that if one grey face is obtained by translating another through an element of $\mathcal{L}$, then their colourings have the same distribution. Such a random colouring $\mathcal{C}$ gives rise to a hyperlattice percolation model $\mathcal{H}(\mathbf{p})$ : simply take the non-crossing partition of a hyperedge $e$ to be the partition of the black faces around the corresponding grey face $F_{e}$ induced by the (black part of) the colouring of $F_{e}$. We say that $\mathcal{C}$ realizes the model $\mathcal{H}(\mathbf{p})$.

It turns out that, at one point in the coming argument, we may need to modify the colouring within certain faces. To enable this, we need our random colouring to satisfy a certain technical assumption. Given a colouring $\chi$ of a grey face $F_{e}$, the colour components of $\chi$ are the maximal connected monochromatic subsets of $F_{e}$.

Definition 5.8. An independent lattice colouring $\mathcal{C}$ is malleable if two conditions hold. First, within each grey face $F_{e}$, the all-white colouring has positive probability. Second, if $\chi$ is a colouring of $F_{e}$ with positive probability, and $\chi^{\prime}$ is obtained from $\chi$ by recolouring a white component black, then $\chi^{\prime}$ has positive probability.

Note that, in a malleable colouring, the all-black colouring of a grey face necessarily has positive probability, so a malleable colouring is non-degenerate 
(meaning, as before, that within each grey face, the all-black and all-white colourings have positive probability).

In general, recolouring as above may change the partition $\pi$ corresponding to the colouring $\chi$ in many different ways. However, if we restrict the colourings suitably, the situation becomes simpler. Let us call a part of a partition $\pi$ nontrivial if it is not a singleton. A colouring $\chi$ of a grey face $F_{e}$ is minimal if its black components are in one-to-one correspondence with the non-trivial parts of $\pi$, and its white components with those of the dual partition $\pi^{*}$. Let us say that a part $P$ of $\pi$ is adjacent to a part $P^{\prime}$ of $\pi^{*}$ if, in the original polygon formulation of non-crossing partitions, $P$ contains a vertex $v$ and $P^{\prime}$ an edge $e$ incident to $v$. This corresponds to $P$ containing a black vertex adjacent to a white dual vertex in $P^{\prime}$. If $\chi$ is a minimal colouring associated to a partition $\pi$, then recolouring a white component black has the effect of uniting all parts of $\pi$ adjacent to some non-trivial part of $\pi^{*}$. We call such an operation on a partition a joining. In the dual, the operation is simpler: simply split a non-trivial part into singletons.

Given a hyperedge $e$, by the top partition of its vertices we mean the partition into a single part. The bottom partition is that into singletons. Recall that a probability vector $\mathbf{p}$ associated to a hyperlattice $(\mathcal{H}, \mathcal{L})$ assigns a probability $p_{i, \pi}$ to each non-crossing partition $\pi$ of the vertices of a hyperedge $e$, where $i$ encodes which orbit of the action of $\mathcal{L}$ the hyperedge $e$ belongs to.

A hyperlattice percolation model $\mathcal{H}(\mathbf{p})$ is non-degenerate if $p_{i, \pi}>0$ whenever $\pi$ is a top or bottom partition.

Definition 5.9. A hyperlattice percolation model $\mathcal{H}(\mathbf{p})$ is malleable if it is non-degenerate and, whenever $p_{i, \pi}>0$ and $\pi^{\prime}$ is obtained from $\pi$ by a joining operation as defined above, then $p_{i, \pi^{\prime}}>0$.

Note that if $\mathcal{H}$ is 3 -uniform, then any non-degenerate model $\mathcal{H}(\mathbf{p})$ is automatically malleable: any joining operation results in the top partition. Also, any $\mathbf{p}$ assigning positive probability to all top and bottom partitions but to no other partitions gives a malleable model: the unique joining operation that may be performed on a bottom partition yields the corresponding top partition. Thus malleability holds automatically in the 'site percolation' models considered in the discussion surrounding Theorem 2.2 .

If $\mathcal{H}(\mathbf{p})$ is self-dual, then malleability is equivalent to its dual formulation, that if $p_{i, \pi}>0$ and $\pi^{\prime}$ is obtained from $\pi$ by splitting a part into singletons, then $p_{i, \pi^{\prime}}>0$.

The next lemma captures the connection between the notions of malleability for probability vectors and for lattice colourings.

Lemma 5.10. Let $\mathcal{H}(\mathbf{p})$ be a malleable hyperlattice percolation model. Then $\mathcal{H}(\mathbf{p})$ may be realized by a malleable independent lattice colouring.

Proof. Regard $\mathcal{H}$ as a 3-coloured cubic map, as usual. For each grey face $F_{e}$ corresponding to hyperedge $e$ with at least 3 vertices, subdivide it into subfaces as in Figure 4.2 (When $|e|=3$ one can also use the simpler subdivision shown 
in Figure 4.1.) It is not hard to check that for any non-crossing partition $\pi$ of the vertices of $e$, there is at least one black/white colouring of the subfaces of $F_{e}$ that gives a minimal colouring realizing the partition $\pi$. If there are $N_{\pi}$ such colourings, assign each probability $p_{i, \pi} / N_{\pi}$, where $i$ is the probability vector entry corresponding to $e$. Since $\mathcal{H}(\mathbf{p})$ is non-degenerate, the all-black and all-white colourings (which are minimal) receive positive probability. Also, if $\chi$ is any colouring of $F_{e}$ receiving positive probability, then $p_{i, \pi}>0$ for the corresponding $\pi$. If $\chi^{\prime}$ is obtained from $\chi$ by recolouring a white component to black, then $\chi^{\prime}$ is minimal, and corresponds to a partition $\pi^{\prime}$ obtained from $\pi$ by a joining operation. Since $\mathcal{H}(\mathbf{p})$ is malleable, we have $p_{i, \pi^{\prime}}>0$, so $\chi^{\prime}$ has positive probability.

For a hyperedge $e$ with $|e|=2$, there is no need to subdivide $F_{e}$ at all; simply colour $F_{e}$ black or white with the appropriate (positive) probabilities. Finally, if $|e|=1$ then the colouring of $F_{e}$ is irrelevant, so we may colour $F_{e}$ black with probability $1 / 2$ and white otherwise.

Of course, a similar but simpler argument shows that any non-degenerate hyperlattice percolation model may be realized by a non-degenerate independent lattice colouring.

As noted in Section 4 a non-degenerate independent lattice colouring $\mathcal{C}$ corresponds to a product probability measure on a power of a certain poset in a natural way; furthermore, the non-degeneracy condition ensures that Lemma 3.2 applies both to this poset and to its reverse. Events defined by the existence of black paths with certain properties are upsets; events defined by the existence of white paths are downsets.

For the rest of this section we fix a malleable independent lattice colouring $\mathcal{C}$. Recall that a black horizontal crossing of a rectangle $R$ is a piecewise-linear path $P$ in the plane joining a point on the left-hand side of $R$ to a point on the right and otherwise lying in the interior of $R$, such that every point of $P$ is black. White vertical crossings are defined similarly, and so on. In our product probability space, events such as $H_{\mathrm{b}}(R)$ are increasing.

We shall use the following lemma, which applies to all black/white colourings of cubic planar maps, i.e., involves no randomness.

Lemma 5.11. Let $R$ be a rectangle in $\mathbb{R}^{2}$ in general position with respect to a colouring $\mathcal{C}$. Then precisely one of the events $H_{\mathrm{b}}(R)$ and $V_{\mathrm{w}}(R)$ holds.

Proof. As in [6, Ch. 8, Lemma 12], from where Figure 5.4 is adapted, re-colour the points outside $R$ as in Figure 5.4. and consider the interfaces between black and white regions.

We shall need the equivalent of highest and lowest open crossings; these are the highest and lowest black crossings of a rectangle, defined below. The definitions require a little care, as the independence properties are not quite what one would like.

Given a rectangle $R$ and our colouring $\mathcal{C}$, shade the outside of the rectangle as in Figure 5.4. Let $I^{+}$be the interface shown by the thick black line, starting 


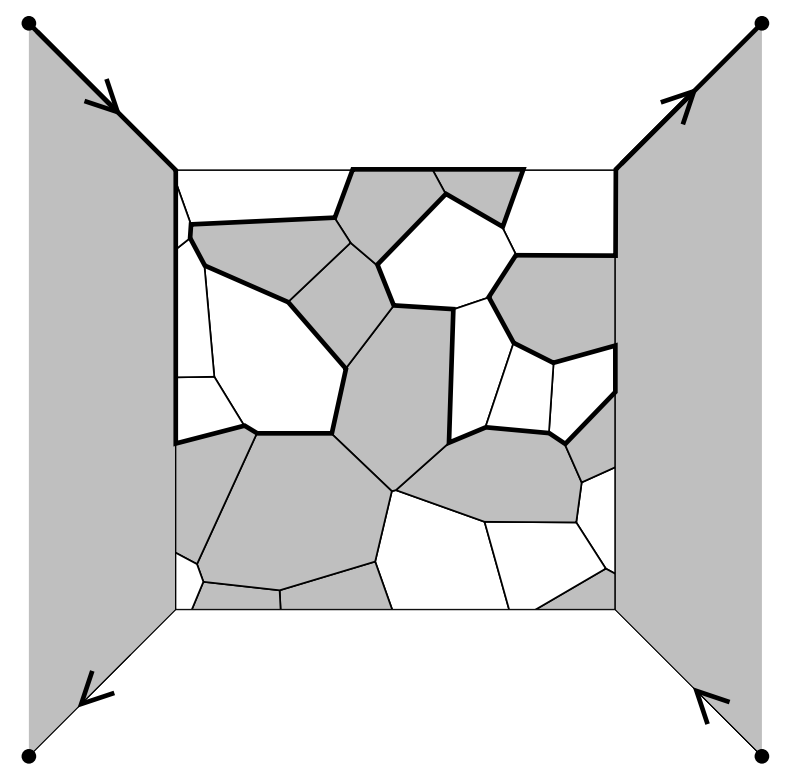

Figure 5.4: The shading inside the (square) rectangle $R$ is from $\mathcal{C}$. The event $H_{\mathrm{b}}(R)$ holds if and only if the outer black regions are joined by a black path, and $V_{\mathrm{w}}(R)$ holds if and only if the outer white regions are joined by a white path. Tracing the interface between black and white regions shows that one of these events must hold.

at the top left corner. Thus if $H_{\mathrm{b}}(R)$ holds, then $I^{+}$leaves the rectangle $R$ from the top right corner. Let $I_{0}^{+}$be the minimal subpath of $I^{+}$meeting both vertical sides of $R$. In the figure, points just to the right of $I^{+}$are black. Hence, points just to the right of $I_{0}^{+}$give a black path within $R$ joining the left to the right. We call this path the highest black horizontal crossing of $R$, and denote it $U H(R)$. Similarly, again assuming $H_{\mathrm{b}}(R)$ holds, the interface $I^{-}$starting at the bottom right corner leaves at the bottom left; we define $I_{0}^{-}$to be the minimal subpath joining the vertical sides of $R$; the points to the right of $I_{0}^{-}$form the lowest black horizontal crossing of $R$, written $L H(R)$. In fact, we can usually work directly with the interfaces $I_{0}^{-}$and $I_{0}^{+}$.

As before, a rectangle $R$ is large if all its sides have length at least $100 d_{0}$, where $d_{0}$ is the constant from Lemma 4.2 .

Given an angle $\theta$, a $\theta$-aligned rectangle $R^{\prime}$, and 'length scales' $m$ and $n$, let $E_{\mathrm{v}}\left(R^{\prime}, m, n, \alpha, \eta\right)$ be the event that $R^{\prime}$ contains a black path $P$ such that, after rotating so that $R^{\prime}$ is horizontal, the endpoints $(x, y)$ and $\left(x^{\prime}, y^{\prime}\right)$ of $P$ satisfy $\left(y^{\prime}-y\right) / n \geq \alpha$ and $\left|x^{\prime}-x\right| / m \leq(1-\eta)\left(y^{\prime}-y\right) / n$.

Our aim now is to adapt the proof of Theorem 5.3 to prove the following result.

Theorem 5.12. Let $\mathcal{C}$ be a malleable independent lattice colouring. Given con- 
stants $\alpha, \beta_{1} \leq 1 / 3, \beta_{2} \leq 2, \eta>3 \beta_{1}$, and $c_{1}, c_{2}>0$, there exists a constant $c>0$ such that the following holds. Suppose that $R$ is a large rectangle with width $m$ and height $n$ and any orientation, and $R^{\prime}$ is a large rectangle with the same orientation, width $\beta_{1} m$, and height $\beta_{2} n$. If $\mathbb{P}\left(H_{\mathrm{b}}(R)\right) \geq c_{1}, \mathbb{P}\left(V_{\mathrm{b}}(R)\right) \geq c_{1}$, and $\mathbb{P}\left(E_{\mathrm{v}}\left(R^{\prime}, m, n, \alpha, \eta\right)\right) \geq c_{2}$, then $\mathbb{P}\left(V_{\mathrm{b}}\left(R^{\prime \prime}\right)\right) \geq c$ for any rectangle $R^{\prime \prime}$ with the same orientation as $R$, width $m$ and height $100 \mathrm{n}$.

Our proof of Theorem 5.12 will follow that of Theorem 5.3 in the previous subsection, rescaling to map the square $S_{1}$ considered there onto the rectangle $R$. Unfortunately, there are various additional complications; for example, we cannot assume that congruent rectangles with the same orientation have the same crossing probabilities. These complications can be dealt with using Corollary 4.10. There will also be some other difficulties.

Remark 5.13. As noted in Section 4, although the probabilities of events such as the existence of various crossings of a rectangle $R$ will depend very much on the orientation of $R$, and to a lesser extent on its position, all the lower bounds we shall prove will depend on $\mathcal{C}$ only via three quantities: the quantity $d_{0}$ appearing in Lemma 4.2 (which provides an upper bound on the diameter of a face), the maximum number $N$ of faces meeting any disk of radius 1 , and the minimum probability $p_{0}$ of a configuration within a face. These three quantities are preserved by rotations and translations, so whenever we consider a single rectangle $R$, we may rotate and translate $R$ and $\mathcal{C}$ together so that $R$ has the form $[0, m] \times[0, n]$.

Let us write $h(R)$ for $\mathbb{P}\left(H_{\mathrm{b}}(R)\right)$ and $v(R)$ for $\mathbb{P}\left(V_{\mathrm{b}}(R)\right)$. Also, we write $h(m, n)$ for the probability that $[0, m] \times[0, n]$ has a black horizontal crossing, and $v(m, n)$ for the probability that it has a black vertical crossing. Recall that by Corollary 4.10 there is an increasing function $\psi:(0,1) \rightarrow(0,1)$ such that if $R$ and $R^{\prime}$ are large rectangles with the same orientation, width and height, then $h\left(R^{\prime}\right) \geq \psi(h(R))$ and $v\left(R^{\prime}\right) \geq \psi(v(R))$.

Recall that the colouring $\mathcal{C}$ we are considering is malleable and hence nondegenerate, meaning that within any grey face, the all-black and all-white colourings have positive probabilities. As noted earlier, non-degeneracy allows us to apply our Harris-type lemma, Lemma 3.2, to two black-increasing events, or two white-increasing events. Throughout the proof of Theorem 5.12 we write $F$ for the function whose existence is guaranteed by Lemma 3.2 , so for any two black-increasing events $A$ and $B$ we have

$$
\mathbb{P}(A \cap B) \geq F(\mathbb{P}(A), \mathbb{P}(B)) .
$$

The first step in our proof of Theorem 5.12 is the analogue of Lemma 5.4 there is an additional assumption (that $h(m, n)$ and $v(m, n)$ are at least $c_{1}$ ) since we do not have the analogue of Lemma 5.1 in this context.

Lemma 5.14. Let $\mathcal{C}$ be a non-degenerate independent lattice colouring, and let $\varepsilon>0, c_{1}>0$ and $c^{\prime}>0$. There is a $c>0$ such that for any $m, n \geq 100 d_{0}$, if $h(m, n) \geq c_{1}, v(m, n) \geq c_{1}$, and $v(m,(1+\varepsilon) n) \geq c^{\prime}$, then $v(m, 100 n) \geq c$. 
Proof. Fix $\varepsilon, c_{1}$ and $c^{\prime}>0$. Let $\alpha_{0}=c_{1}$; for $i \geq 0$ let

$$
\alpha_{i+1}=F\left(F\left(\alpha_{i}, \psi\left(c_{1}\right)\right), \psi\left(c^{\prime}\right)\right),
$$

where $\psi$ is the function appearing in Corollary 4.10, and set $c=\alpha_{\lceil 99 / \varepsilon\rceil}$. Note that $c>0$.

Suppose that $m, n \geq 100 d_{0}, h(m, n) \geq c_{1}, v(m, n) \geq c_{1}$, and $v(m,(1+\varepsilon) n) \geq$ $c^{\prime}$. We claim that for every $i \geq 0$ we have

$$
v(m,(1+i \varepsilon) n) \geq \alpha_{i} .
$$

Setting $i=\lceil 99 / \varepsilon\rceil$, we then have $1+i \varepsilon \geq 100$, so $v(m, 100 n) \geq v(m,(1+i \varepsilon) n) \geq$ $c$, and the result follows.

We prove (15) by induction. For $i=0$ it is true by assumption. Turning to the induction step, suppose that (15) holds for some $i$, and consider the rectangles $R_{1}=[0, m] \times[0,(1+i \varepsilon) n], R_{2}=[0, m] \times[i \varepsilon n,(1+i \varepsilon) n]$, and $R_{3}=$ $[0, m] \times[i \varepsilon n, 1+(i+1) \varepsilon n]$, as in Figure [5.5. Let $R_{2}^{\prime}=[0, m] \times[0, n]$ and $R_{3}^{\prime}=[0, m] \times[0,(1+\varepsilon) n]$, so $R_{2}^{\prime}$ and $R_{3}^{\prime}$ are images of $R_{2}$ and $R_{3}$ under appropriate translations.

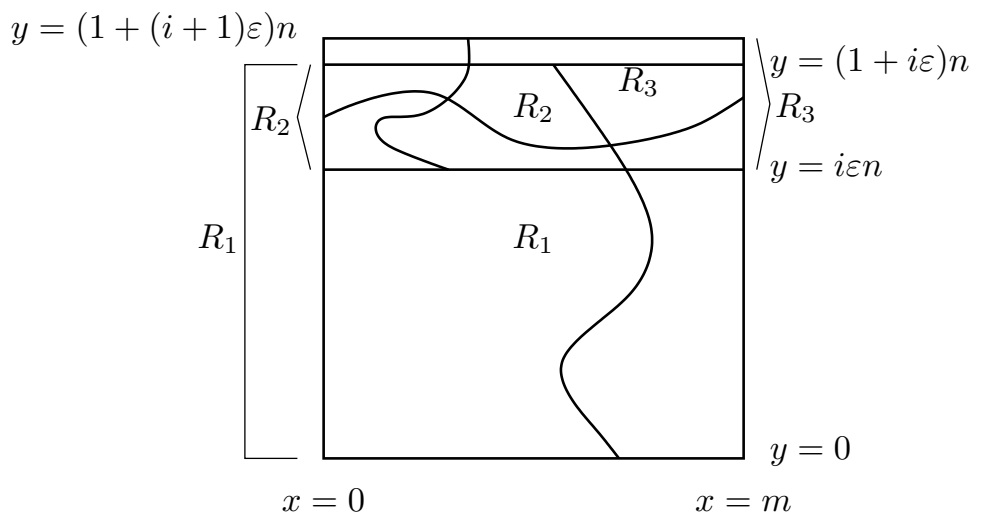

Figure 5.5: The rectangles $R_{1}$ and $R_{3}$, with their intersection $R_{2}$. Whenever $R_{1}$ and $R_{3}$ have black vertical crossings and $R_{2}$ has a black horizontal crossing, then these crossings can be combined to form a black vertical crossing of $R_{1} \cup R_{3}$.

By the induction hypothesis we have $\mathbb{P}\left(V_{\mathrm{b}}\left(R_{1}\right)\right)=v(m,(1+i \varepsilon) n) \geq \alpha_{i}$ and by assumption we have $\mathbb{P}\left(H_{\mathrm{b}}\left(R_{2}^{\prime}\right)\right)=h(m, n) \geq c_{1}$. Hence, by Corollary 4.10, we have $\mathbb{P}\left(H_{\mathrm{b}}\left(R_{2}\right)\right) \geq \psi\left(c_{1}\right)$. Similarly, by assumption $\mathbb{P}\left(V_{\mathrm{b}}\left(R_{3}^{\prime}\right)\right)=v(m,(1+$ $\varepsilon) n) \geq c^{\prime}$, so $\mathbb{P}\left(V_{\mathrm{b}}\left(R_{3}\right)\right) \geq \psi\left(c^{\prime}\right)$.

Since $V_{\mathrm{b}}\left(R_{1}\right)$ and $H_{\mathrm{b}}\left(R_{2}\right)$ are increasing events, from (14) it follows that $\mathbb{P}\left(V_{\mathrm{b}}\left(R_{1}\right) \cap H_{\mathrm{b}}\left(R_{2}\right)\right) \geq F\left(\alpha_{i}, \psi\left(c_{1}\right)\right)$. Applying (14) to the increasing events $V_{\mathrm{b}}\left(R_{1}\right) \cap H_{\mathrm{b}}\left(R_{2}\right)$ and $V_{\mathrm{b}}\left(R_{3}\right)$, it follows that with probability at least $\alpha_{i+1}$, the events $V_{\mathrm{b}}\left(R_{1}\right), H_{\mathrm{b}}\left(R_{2}\right)$ and $V_{\mathrm{b}}\left(R_{3}\right)$ all hold. Choosing black paths $P_{1}, P_{2}$ and $P_{3}$ witnessing these events, $P_{2}$ meets both $P_{1}$ and $P_{3}$, and it follows that 
$V_{\mathrm{b}}\left(R_{1} \cup R_{3}\right)$ holds. Hence

$$
v(m,(1+(i+1) \varepsilon) n)=\mathbb{P}\left(V_{\mathrm{b}}\left(R_{1} \cup R_{3}\right)\right) \geq \alpha_{i+1},
$$

proving the induction step and so completing the proof of (15).

The next step is the analogue of Lemma 5.5 this concerns crossings of nearby squares (now rectangles) that are joined. As in the previous lemma, we work with rectangles, not squares, and add the assumptions that $h(m, n)$ and $v(m, n)$ are at least some constant $c_{1}$ rather than appeal to Lemma 5.1. The definition of the event $J$ is as before, mutatis mutandis: specifically, wherever $n$ appears in the definition, it is replaced by $m$ or $n$ depending on whether we are considering an $x$-coordinate or a $y$-coordinate.

Lemma 5.15. Let $\mathcal{C}$ be a non-degenerate independent lattice colouring. For any $\varepsilon>0, c_{1}>0$ and $c^{\prime}>0$, there is a $c>0$ such that for any $m, n \geq 100 d_{0}$ with $h(m, n), v(m, n) \geq c_{1}$, if there exist $m$-by-n rectangles $R_{1}$ and $R_{2}$, with $R_{2}$ obtained by translating $R_{1}$ upwards by a distance of $\varepsilon n$, for which $\mathbb{P}\left(J\left(R_{1}, R_{2}\right)\right) \geq$ $c^{\prime}$, then $v(m, 100 n) \geq c$.

Proof. Modify the proof of Lemma 5.5 as above: replace each application of Harris's Lemma by an appeal to (14), and use the fact that, under our assumptions, any $m$-by- $n$ rectangle $R$ has $h(R), v(R) \geq \psi\left(c_{1}\right)>0$ by Corollary 4.10 .

Next comes the analogue of Lemma [5.6. For two $m$-by-n rectangles $R_{1}$ and $R_{2}$, with $R_{2}$ obtained by translating $R_{1}$ upwards by a distance of $\varepsilon n / 10$, define the strip $T=T\left(R_{1}, R_{2}\right)$ to be the region between the vertical lines containing the vertical sides of $R_{1}$ and $R_{2}$, and let $G_{\varepsilon}\left(R_{1}, R_{2}\right)$ be the event that $H_{\mathrm{b}}\left(R_{1}\right)$ and $H_{\mathrm{b}}\left(R_{2}\right)$ hold, the path $P_{1}=L H\left(R_{1}\right)$ is below $P_{2}=U H\left(R_{2}\right)$ in $T$, and the area of $T$ between $P_{1}$ and $P_{2}$ is at most $\varepsilon m n$.

Lemma 5.16. Let $\mathcal{C}$ be a non-degenerate independent lattice colouring. For any $\varepsilon>0$ and $c_{1}>0$ there are constants $c_{3}>0$ and $c>0$ such that for any $m, n \geq 100 d_{0}$ with $h(m, n), v(m, n) \geq c_{1}$, either there exist $m$-by- $n$ rectangles $R_{1}$ and $R_{2}$ as above with $\mathbb{P}\left(G_{\varepsilon}\left(R_{1}, R_{2}\right)\right) \geq c_{3}$, or $v(m, 100 n)>c$.

Proof. Imitate the proof of Lemma 5.6, mutatis mutandis.

In adapting the main part of the proof of Theorem 5.3 there is only one genuine additional complication: we shall have to work to join up paths in our two configurations $\omega_{1}$ and $\omega_{2}^{X}$. The other changes are mostly in notation.

Recall that our independent lattice colouring $\mathcal{C}$ is obtained by randomly colouring the subdivided grey faces of the 3 -coloured planar map $\mathcal{H}$. As before, let $\Omega$ denote the set of all configurations, i.e., assignments of states to the grey faces of $\mathcal{H}$. Let $\mathbb{A}$ be an algorithm that examines the states of grey faces in its input configuration one by one, with the next face to be examined determined by the states of the faces examined so far. Assume that $\mathbb{A}$ terminates and write $\mathcal{S}_{\mathbb{A}}(\omega)$ for the set of grey faces examined by $\mathbb{A}$ when run on the configuration $\omega$. Recalling that $\mathcal{H}$ is invariant under translations through elements of the 
lattice $\mathcal{L}$, define a function $f_{\mathbb{A}}$ from $\Omega^{2} \times \mathcal{L}$ to $\Omega$ by $\left(\omega_{1}, \omega_{2}, \ell\right) \mapsto \omega$, where $\omega=f_{\mathbb{A}}\left(\omega_{1}, \omega_{2}, \ell\right)$ is the configuration given by

$$
\omega(g)=f_{\mathbb{A}}\left(\omega_{1}, \omega_{2}, \ell\right)(g)= \begin{cases}\omega_{1}(g) & \text { if } g \in \mathcal{S}_{\mathbb{A}}\left(\omega_{1}\right), \\ \omega_{2}(g-\ell) & \text { if } g \notin \mathcal{S}_{\mathbb{A}}\left(\omega_{1}\right) .\end{cases}
$$

Here $g$ denotes an arbitrary grey face, and $g-\ell$ the grey face obtained by translating $g$ through the vector $-\ell$. In other words, as before, the state of a grey face in $\omega=f_{\mathbb{A}}\left(\omega_{1}, \omega_{2}, \ell\right)$ is given either by its state in $\omega_{1}$ or by its state in the translate $\omega_{2}^{\ell}$ of $\omega_{2}$, according to whether or not the algorithm $\mathbb{A}$ examines $g$ when run on the configuration $\omega_{1}$.

Let $\mathcal{L}_{m, n}$ be the set of points of $\mathcal{L}$ in $[-5 m, 5 m] \times[-5 n, 5 n]$, and interpret $\Omega^{2} \times \mathcal{L}_{m, n}$ as the product probability space in which the two configurations have the distribution associated to $\mathcal{C}$ and are independent, and the random vector $X \in \mathcal{L}_{m, n}$ is chosen uniformly from $\mathcal{L}_{m, n}$. As before, $\omega=f_{\mathbb{A}}\left(\omega_{1}, \omega_{2}, X\right)$ has the distribution appropriate for $\mathcal{C}$.

Proof of Theorem 5.12. We follow the proof of Theorem 5.3. concentrating on the differences. In the light of Remark 5.13, rotating the rectangles under consideration together with $\mathcal{C}$, we may assume that $R$ and $R^{\prime}$ are aligned with the coordinate axes, i.e., are 0 -aligned.

First, note that (7) now holds by assumption. As before, choose $\gamma>0$ such that (8) holds, and then choose $\varepsilon>0$ such that $\varepsilon<\gamma$ and (9) holds. Let $m$ and $n$ satisfy the assumptions of the theorem. Then, since $h(m, n), v(m, n) \geq c_{1}$, we can apply Lemma 5.16. Let $c_{3}$ and $c$ be the constants given by Lemma 5.16. so either $v(m, 100 n) \geq c$, in which case we are done, or there are $m$-by- $n 0$-aligned rectangles $S_{1}$ and $S_{2}$ with $S_{2}$ obtained by translating $S_{1}$ upwards by a distance of $\varepsilon n / 10$ such that

$$
\mathbb{P}\left(G_{\varepsilon}\left(S_{1}, S_{2}\right)\right) \geq c_{3} .
$$

We may assume that the second case holds. Translating the rectangles under consideration together with $\mathcal{C}$, we may assume that $S_{1}=[0, m] \times[0, n]$ and $S_{2}=[0, m] \times[\varepsilon n / 10,(1+\varepsilon / 10) n]$.

As before, we explore $S_{1}$ from below to find its lowest black horizontal crossing $P_{1}$, if it exists, and $S_{2}$ from above to find its highest black horizontal crossing $P_{2}$. More precisely, we let $I_{1}$ be the interface $I_{0}^{-}$in $S_{1}$ described earlier in the subsection, and $I_{2}$ the interface $I_{0}^{+}$in $S_{2}$. Note that orienting these interfaces as in Figure 5.6 the points just to the right of each interface are black while those to the left are either white, or inside $T$ but outside the relevant rectangle $S_{i}$ vertically, if the interface runs along the top/bottom of $S_{i}$. It follows that $I_{1}$ and $I_{2}$ cannot meet. Our definitions allow us to view $I_{i}$ as a black path itself, although it is perhaps clearer to think of $P_{i}$ as running next to $I_{i}$. In the present context, the gap $G$ is the region between $I_{1}$ and $I_{2}$; we define $A$ and $B$ to be the regions of our strip $[0, m] \times \mathbb{R}$ below $I_{1}$ and above $I_{2}$, respectively.

As before, we first test whether $\omega_{1} \in G_{\varepsilon}$, using the algorithm $\mathbb{A}$ implicitly defined above. More precisely, we implement $\mathbb{A}$ by following the full interfaces $I^{-}$and $I^{+}$in the relevant rectangles. Note that this only involves 'testing' the 


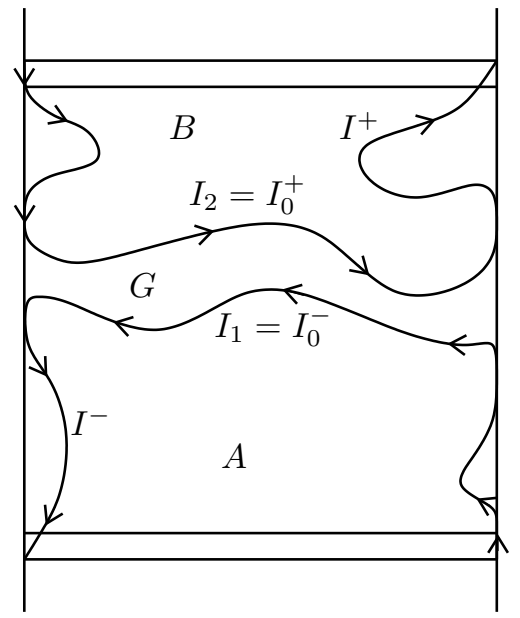

Figure 5.6: The overlapping congruent rectangles $S_{1}$ (below) and $S_{2}$ (above), with the interfaces $I^{-}$in $S_{1}$ and $I^{+}$in $S_{2}$ defined as in Figure 5.4 $I_{0}^{-}$and $I_{0}^{+}$ are the minimal subpaths of $I^{-}$and $I^{+}$crossing the strip $S_{1} \cup S_{2}$. Note that every point just to the right of $I^{ \pm}$is black or outside $S_{1} \cup S_{2}$ horizontally; points just to the right of $I_{0}^{ \pm}$are always inside $S_{1} \cup S_{2}$ and hence black. Points to the left of $I^{ \pm}$are white or outside $S_{1} \cup S_{2}$ vertically. The 'gap' $G$ is the region between $I_{1}=I_{0}^{-}$and $I_{2}=I_{0}^{+}$.

state of grey faces $f$ that meet one of these interfaces, where $f$ meets $I$ if $I$ passes through $f$, or along one of the sides of $f$. It is easy to check that all of $I^{-}$lies below $I_{0}^{-}=I_{1}$, and all of $I^{+}$above $I_{2}$. Let $Z\left(I_{i}\right)$ denote the union of $I_{i}$ and all grey faces that meet $I_{i}$, which we think of as the zone of influence of $I_{i}$. Then when $G_{\varepsilon}$ holds, the algorithm $\mathbb{A}$ establishes this by looking only at grey faces in $A \cup B \cup Z\left(I_{1}\right) \cup Z\left(I_{2}\right)$. Defining $\omega=f_{\mathbb{A}}\left(\omega_{1}, \omega_{2}, X\right)$ as before, it follows that any grey face contained in $G \backslash\left(Z\left(I_{1}\right) \cup Z\left(I_{2}\right)\right)$ has its state in $\omega$ given by its state in $\omega_{2}^{X}$, the configuration $\omega_{2}$ translated through the random vector $X$.

As before, we condition on $\omega_{1}$, assuming that $G_{\varepsilon}$ holds, which it does with probability bounded away from zero. Furthermore, we condition on $\omega_{2}$, assuming the existence of a path $P$ with the property described in the definition of $E_{\mathrm{v}}\left(R^{\prime}, m, n, \alpha, \eta\right)$. Again, the probability of this event is bounded away from zero, this time by assumption. As before, the only remaining randomness is in the choice of the random translation $X$.

Recalling that we may take $m$ and $n$ large, apart from one very minor technical issue that we postpone to the end of the proof, trivial modifications to our previous arguments show that with probability bounded away from zero, the random translate $P+X$ of $P$ crosses from $A$ to $B$, while remaining within the strip $T$. In fact, adjusting the constants slightly if necessary, we can assume that it does not come within distance $d_{0}$ of the edges of $T$.

Let $E \subset \Omega^{2} \times \mathcal{L}_{m, n}$ denote the set of triples $\left(\omega_{1}, \omega_{2}, \ell\right)$ such that $\omega_{1} \in G_{\varepsilon}$, and 
$\omega_{2}$ contains a path $P$ as above whose translate $P+\ell$ meets the interfaces $I_{1}$ and $I_{2}$ in $\omega_{1}$. We have shown that $\mathbb{P}(E)$ is bounded away from zero. Unfortunately, unlike in the $\mathbb{Z}^{2}$ setting, it is not true that if $\left(\omega_{1}, \omega_{2}, \ell\right) \in E$, then $f_{\mathbb{A}}\left(\omega_{1}, \omega_{2}, \ell\right) \in$ $J$; the problem is illustrated in Figure 5.7 .

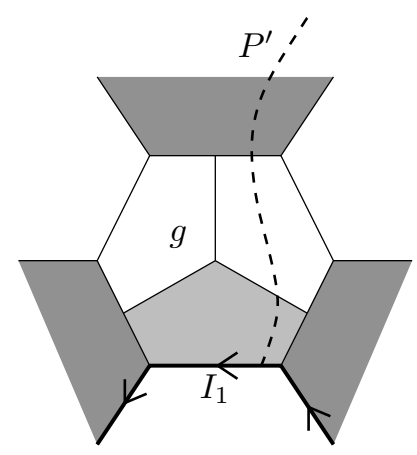

Figure 5.7: A hexagonal grey face $g$ surrounded by black and white faces of $\mathcal{H}$. In the random colouring associated to $\omega_{1}$, the internal colouring of $g$ is indicated by the lighter shading. In this case $I_{1}$ touches $g$. The dashed line shows a black path $P^{\prime}$ in the configuration $\omega_{2}^{X}$; since the state of $g$ is read from $\omega_{1}$ (even though $g$ is in the 'gap'), in the combined configuration $\omega$, the path $P^{\prime}$ fails to join up with $I_{1}$.

To overcome this problem, we adjust the colourings of at most two faces. Let $\left(\omega_{1}, \omega_{2}, \ell\right) \in E$, and let $P^{\prime}$ denote a minimal part of the path $P+\ell$ joining $I_{1}$ to $I_{2}$. Then $P^{\prime}$ necessarily lies in the gap $G$. Let $P^{\prime \prime}$ denote a minimal subpath of $P^{\prime}$ joining $Z\left(I_{1}\right)$ to $Z\left(I_{2}\right)$, and let $v_{1}$ and $v_{2}$ denote its endpoints (which may coincide, if $Z\left(I_{1}\right)$ and $Z\left(I_{2}\right)$ meet); see Figure 5.8. Note that any interior point of $P^{\prime \prime}$ lies in $G \backslash\left(Z\left(I_{1}\right) \cup Z\left(I_{2}\right)\right)$, so its colour in $\omega=f_{\mathbb{A}}\left(\omega_{1}, \omega_{2}, \ell\right)$ is its colour in $\omega_{2}^{\ell}$; since $P^{\prime \prime} \subset P+\ell$, such points are therefore black.

Now each $v_{i}$ is either on $I_{i}$, or belongs to a grey face $f_{i}$ which meets $I_{i}$. Suppose the latter case holds for $i=1,2$. To handle the remaining cases we simply recolour at most one face in the following argument, rather than two. Recalling that $P+\ell$ does not pass within distance $d_{0}$ of the edges of $T$, note that each $f_{i}$ lies entirely with the strip $T$. Let $\omega_{1}^{\prime}$ be the configuration obtained from $\omega_{1}$ by recolouring all points of $\left(f_{1} \cup f_{2}\right) \cap G$ black. As we shall show in a moment, this is a legal configuration. Assuming this for the moment, if we restrict our attention overall to a finite region of the plane, as we may, the ratio $\mathbb{P}\left(\omega_{1}^{\prime}\right) / \mathbb{P}\left(\omega_{1}\right)$ of the probabilities of the individual configurations is bounded below by $p_{0}^{2}$, where $p_{0}>0$ is the minimum probability of any possible state of a grey face.

The key point is that recolouring a set of points within the gap $G$ black does not change the interfaces $I_{1}$ and $I_{2}$; these interfaces are determined by the full interfaces $I^{ \pm}$shown in Figure [5.6. which are defined 'locally', and the side of $I_{i}$ on which the gap lies is already 'locally black'. Thus the algorithm $\mathbb{A}$ examines 


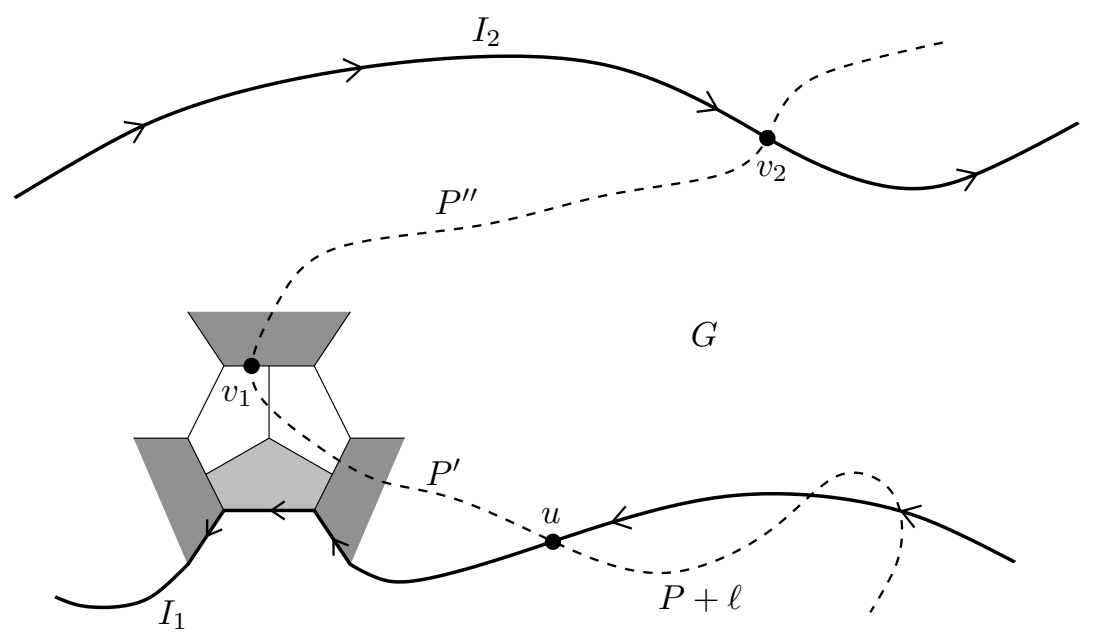

Figure 5.8: The path $P+\ell$ (dashed line) crossing the gap $G$, from below $I_{1}$ to above $I_{2}$. The subpath $P^{\prime}$ of $P+\ell$, here from $u$ to $v_{2}$, is minimal subject to crossing $G$, and so lies within $G$. In turn, $P^{\prime \prime}$, from $v_{1}$ to $v_{2}$, is minimal subject to joining $Z\left(I_{1}\right)$ to $Z\left(I_{2}\right)$. In this example, $v_{2}$ is on $I_{2}$, while $v_{1}$ is in a grey face $f_{1}$ (the hexagon in the figure) touching $I_{1}$; the two white subfaces inside $f_{1}$ will be recoloured black.

the states of the same set of faces if run on $\omega_{1}^{\prime}$ or on $\omega_{1}$. Recalling that the states of $f_{1}$ and $f_{2}$ in $\omega$ are those in $\omega_{1}$, it follows that in the configuration $\omega^{\prime}=f_{\mathbb{A}}\left(\omega_{1}^{\prime}, \omega_{2}, \ell\right)$ there is a black path joining $I_{1}$ to $I_{2}$, given by the union of $P^{\prime \prime}$ and two short paths from $v_{i}$ to $I_{i}$ within $f_{i} \cap G$. Hence $\omega^{\prime} \in J$.

Let $g: E \rightarrow \Omega^{2} \times \mathcal{L}_{m, n}$ denote the map $\left(\omega_{1}, \omega_{2}, \ell\right) \mapsto\left(\omega_{1}^{\prime}, \omega_{2}, \ell\right)$, and let $E^{\prime}=g(E)$. We have shown that $E^{\prime} \subset f_{\mathbb{A}}^{-1}(J)$. Recalling that $f_{\mathbb{A}}$ is measure preserving, it follows that $\mathbb{P}(J) \geq \mathbb{P}\left(E^{\prime}\right)$. Since our recolouring does not change the interfaces $I_{1}$ and $I_{2}$, given some $\left(\tilde{\omega}, \omega_{2}, \ell\right) \in E^{\prime}$ known to be the image of some unknown $\left(\omega_{1}, \omega_{2}, \ell\right)$ under $g$, we can read off the interfaces $I_{1}$ and $I_{2}$ (defined in $\omega_{1}$ ) by looking at $\tilde{\omega}$. We also know the path $P$ from $\omega_{2}$. This allows us to determine $P^{\prime}$ and $P^{\prime \prime}$ as defined above, and hence $f_{1}$ and $f_{2}$. In other words, we know which two (or at most two) faces were recoloured, though not how. It follows that $g^{-1}\left(\left\{\left(\tilde{\omega}, \omega_{2}, \ell\right)\right\}\right)$ consists of a bounded number of configurations, each of whose probabilities is at most $p_{0}^{-2}$ times that of $\left(\tilde{\omega}, \omega_{2}, \ell\right)$. Hence there is a constant $C$ such that $\mathbb{P}\left(g^{-1}\left(E^{\prime}\right)\right) \leq C \mathbb{P}\left(E^{\prime}\right)$. Since $g^{-1}\left(E^{\prime}\right)=g^{-1}(g(E)) \supset E$, it follows that $\mathbb{P}\left(E^{\prime}\right) \geq \mathbb{P}(E) / C$. Since $E$ is known to have probability bounded away from zero, the result follows.

It remains to establish that the recolouring is permissible, i.e., to show that if we recolour within a grey face $g$ (either $f_{1}$ or $f_{2}$ above), the new colouring has positive probability; this is where we use the assumption that $\mathcal{C}$ is malleable (a condition of Theorem 5.12). Now $G$ is bounded by interfaces running between black and white regions, as well as the sides of the strip $T$; the latter do not meet 
$g$. It follows that $g \cap G$ is the union of one or more colour components (maximal connected monochromatic subsets) of $g$. Our recolouring thus recolours one or more white components within $g$ to black; the definition of malleability ensures that the resulting colouring has positive probability.

Finally, let us comment on the technical issue we overlooked, which is that since the endpoints of $P$ may not differ by a lattice element, we cannot exactly join up translates of $P$ though lattice elements to form $P^{*}$. One way to handle this is to find a short (length $O(1)$ ) black path $P^{\prime}$ in $\omega_{2}$ joining appropriate points within a fundamental domain of $\mathcal{L}$, and form $P^{*}$ by chaining together alternate copies of $P$ and $P^{\prime}$, as described in Remark 5.7 at the end of Subsection 5.1 Alternatively, simply leave small (length at most $d_{0}=O(1)$ ) gaps between the translates of $P$ making up $P^{*}$ : when colouring $R_{0}$, assign colour 0 to any point $v$ such that in the copy of $P^{*}$ starting at $v$, one or more of these small gaps meets $G$. It remains the case that few points receive colour 0: the set of such points is contained in the union of $I=O(1)$ translates of the $d_{0}$-neighbourhood $G^{d_{0}}$ of $G$. Since $G$ is made up of faces whose size is bounded below, the area of $G^{d_{0}}$ is at most a constant times that of $G$, so the area receiving colour 0 is still $O\left(\varepsilon n^{2}\right)$; choosing $\varepsilon$ small enough, the rest of the argument is unchanged.

\subsection{A stronger rectangle-crossing lemma}

Although technically we can do without it, we now present a more convenient version of Theorem 5.12 giving the same conclusion under a weaker assumption. First we need a simple geometric lemma.

By the displacement of a path $P$ we mean the Euclidean distance from its start to its endpoint. By the direction of $P$ we mean the direction from its start to its endpoint, considered as an angle modulo $\pi$. The angle between two paths is simply the (unsigned) angle between their directions, taken as a real number between 0 and $\pi / 2$.

Lemma 5.17. Given $\delta>0$ there exists a constant $C=C(\delta)$ such that whenever $P_{1}$ and $P_{2}$ are two paths in $[0,1 / 10]^{2}$ with displacement at least $\delta$ such that the angle between $P_{1}$ and $P_{2}$ is at least $\delta$, then we can chain together at most $C$ paths each of which is a translate of $P_{1}$ or $P_{2}$ to form a path $P^{*}$ that lies within $[0.1,0.9] \times[-0.5,1.5]$, starts below $y=-0.1$, and ends above $y=1.1$.

Proof. Let $v_{i}$ be the vector from the start of $P_{i}$ to its end, and let $\mathcal{L}$ be the lattice $\left\{a v_{1}+b v_{2}: a, b \in \mathbb{Z}\right\}$. Let $D$ be the fundamental domain of $\mathcal{L}$ whose corners are the origin, $v_{1}, v_{2}$ and $v_{1}+v_{2}$. Note that the area of $D$ is bounded below by a constant depending on $\delta$.

The idea is simply to approximate the line-segment $L$ joining the points $(0.5,-0.3)$ and $(0.5,1.3)$ by a path $Q$ in the graph associated to $\mathcal{L}$ where two lattice points are neighbours if they differ by $\pm v_{i}$ for some $i$; then replace each edge by an appropriate translate of $P_{i}$. We can find such a path $Q$ so that every point of $Q$ is in the same domain $D+a v_{1}+b v_{2}$ as some point of $L$, and $Q$ starts and ends in the same domains as the start and end of $L$; to see this, 
simply apply an affine transformation mapping $\mathcal{L}$ to the usual square grid, and approximate the image of $L$ by an appropriate path.

Since any two points of $D$ have $x$-coordinates that differ by at most $2 / 10$ and $y$-coordinates that differ by at most $2 / 10$, the path $P^{*}$ obtained from $Q$ has the required properties.

Theorem 5.18. Let $\mathcal{C}$ be a malleable independent lattice colouring, and let $\delta$, $c_{1}$ and $c_{2}$ be positive constants. Then there exists a constant $c>0$ such that the following holds. Suppose that $R$ is a large rectangle with width $m$, height $n$ and any orientation, and $R^{\prime}$ is a large rectangle with the same orientation, width $m / 10$, and height $n / 10$. Let $E$ be the event that $R^{\prime}$ contains two black paths such that their images under the affine transformation mapping $R^{\prime}$ to $[0,1 / 10]^{2}$ satisfy the conditions of Lemma 5.17. If $\mathbb{P}\left(H_{\mathrm{b}}(R)\right) \geq c_{1}, \mathbb{P}\left(V_{\mathrm{b}}(R)\right) \geq c_{1}$, and $\mathbb{P}(E) \geq c_{2}$, then $\mathbb{P}\left(V_{\mathrm{b}}\left(R^{\prime \prime}\right)\right) \geq c$ for any rectangle $R^{\prime \prime}$ with the same orientation as $R$, width $m$ and height 100 n.

Proof. The proof is the same as that of Theorem 5.12, except that we construct the 'virtual' path $P^{*}$ as the union of translates of our black paths $P_{1}$ and $P_{2}$ in $\omega_{2}$ given by Lemma 5.17. As before, since we can only translate by elements of the lattice $\mathcal{L}$ of symmetries of $\mathcal{C}$, we may need additional short paths to join up these translates. Each of these additional paths lies within a fundamental domain of $\mathcal{L}$, and we may take them to be translates of appropriate short black paths $P_{3}, \ldots$ in $\omega_{2}$. (With extremely high probability, such paths will exist; we only need one of the many domains to have the property that every grey face meeting it is coloured black.)

Arguing as before, after conditioning on $\omega_{1}$ and $\omega_{2}$, we find that with probability bounded away from zero our random translate of one of the $P_{i}$ joins $I_{1}$ to $I_{2}$, and the rest of the proof is as before.

The precise constants appearing in Lemma 5.17 are not important; the key point is that we can chain together translates of our paths $P_{i}$ to give a path $P^{*}$ with the properties discussed in Remark 5.7. After appropriate rescaling, these properties are that $P^{*}$ crosses a square from bottom to top, with some 'elbow room', i.e., starting well below the bottom and ending well above the top, without coming too close to the vertical sides.

\section{Self-duality and rectangle crossings}

Our aim in this section is to show that if $\mathcal{C}$ is a malleable independent lattice colouring associated to a self-dual plane hyperlattice $\mathcal{H}$, then an analogue of Theorem 5.3 holds for $\mathcal{C}$. It turns out that, due to the lack of symmetry, we cannot specify in advance the orientation of the rectangles we work with. In fact, we cannot even fix their aspect ratio.

Recall that $h_{\theta}(m, n)$ and $v_{\theta}(m, n)$ denote $\mathbb{P}\left(H_{\mathrm{b}}(R)\right)$ and $\mathbb{P}\left(V_{\mathrm{b}}(R)\right)$, where $R$ is an $m$-by- $n \theta$-aligned rectangle centred on the origin. 
Definition 6.1. Let $\rho>1$. We say that a lattice colouring $\mathcal{C}$ has the "large rectangles' property $L R_{\rho}$ if there exists a constant $c>0$ such that for all $L$ and for all large enough $A$ (depending on $L$ ) there are $m, n \geq L$ with $m n=A$ and an angle $\theta$ such that $h_{\theta}(\rho m, n) \geq c$ and $v_{\theta}(m, \rho n) \geq c$.

In symbols, this large rectangles property with parameter $\rho$ may be stated as follows:

$$
\begin{aligned}
\exists c>0 \forall L \exists A_{0} \forall A \geq & A_{0} \exists m, n, \theta: \\
& m, n \geq L, m n=A, h_{\theta}(\rho m, n) \geq c \text { and } v_{\theta}(m, \rho n) \geq c .
\end{aligned}
$$

Roughly speaking, the idea is that we can find a rectangle $R$ so that when we extend it by a factor of $\rho$ horizontally or vertically, the probability of a black horizontal or vertical crossing, respectively, is not too small. We can take this rectangle to have any given large area, and can assume that both sides are at least any given length. However, the orientation and aspect ratio cannot be specified in advance.

Recall that $\mathcal{C}$ is non-degenerate if, within each grey face, the all-black and all-white colourings have positive probability. Under this assumption, one can adapt the usual argument from Harris's Lemma to show that for any $\rho_{1}, \rho_{2}>1$, the property $L R_{\rho_{1}}$ implies $L R_{\rho_{2}}$; the argument is as for Lemma 5.14. In the light of this, the following definition makes sense.

Definition 6.2. A non-degenerate independent lattice colouring $\mathcal{C}$ has the large rectangles property if it has the property $L R_{\rho}$ for some $\rho>1$, and hence for all $\rho>1$.

Recall that $\mathcal{C}$ is malleable if it is non-degenerate and satisfies certain technical extra conditions; for the full definition see Subsection 5.2. Our aim in this section is to prove the following result; as we shall see in the next section, it is then easy to deduce Theorems 2.1 and 2.2 .

Theorem 6.3. Let $\mathcal{C}$ be a malleable independent lattice colouring realizing an (approximately) self-dual hyperlattice percolation model $\mathcal{H}(\mathbf{p})$. Then $\mathcal{C}$ has the large rectangles property.

The proof of Theorem 6.3 will require a little preparation. First, let us restate the property slightly. Given an ellipse $E$ with centre $x_{0}$, let $2 E / 3$ denote the ellipse $\left\{x_{0}+2\left(x-x_{0}\right) / 3: x \in E\right\}$ obtained by shrinking $E$ by a factor of $3 / 2$, keeping the centre the same. Let $E^{0}$ denote the 'annulus' between $E$ and $2 E / 3$, and let $O_{\mathrm{b}}(E)$ denote the event that there is a closed black path in $E^{0}$ surrounding the central hole.

Definition 6.4. A lattice colouring $\mathcal{C}$ has the large ellipses property if there exists a constant $c>0$ such that for all $L$ and for all large enough $A$ (depending on $L$ ) there is an ellipse $E$ centred on the origin with area $A$ and with both axes having length at least $L$ such that $\mathbb{P}\left(O_{\mathrm{b}}(E)\right) \geq c$. 
It is easy to see that the large rectangles property and the large ellipses property are equivalent.

Lemma 6.5. A non-degenerate independent lattice colouring $\mathcal{C}$ has the large rectangles property if and only if it has the large ellipses property.

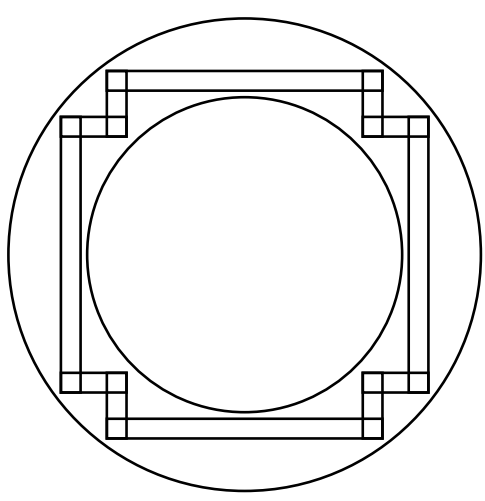

(a)

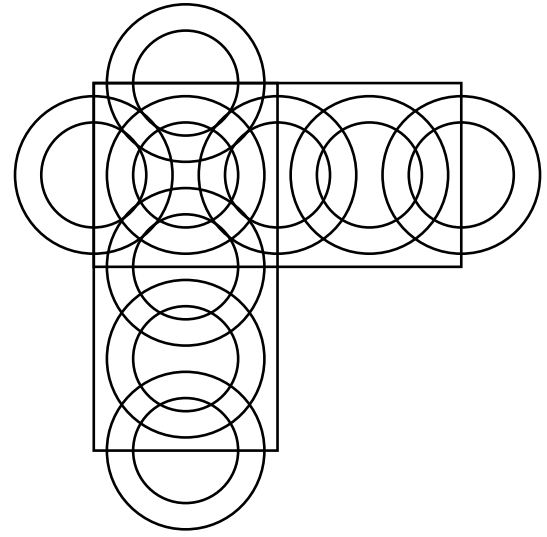

(b)

Figure 6.1: (a) Rectangles in an annulus, and (b) circles crossing two rectangles.

Proof. Suppose first that $\mathcal{C}$ has the large rectangles property; in particular, it has the property $L R_{20}$.

Consider the arrangement of overlapping rectangles shown in Figure 6.1(a). Taking the shorter side of each rectangle to have length 1, the arrangement is such that the longer side of each rectangle has length at most 20. Also, if each rectangle has a black crossing in the long direction, then $O_{\mathrm{b}}(C)$ holds, where $C$ is the outer circle. Let $a$ denote the area of $C$, so $a$ is an absolute constant.

We must show the existence of an ellipse $E$ with (large enough) area $A$ and both axes at least $L$ such that $\mathbb{P}\left(O_{\mathrm{b}}(E)\right) \geq c_{1}$, for some constant $c_{1}$. Let $d_{0}=d_{0}(\mathcal{C})$ be the constant in Lemma 4.2. The large rectangles property $L R_{20}$ gives us a $\theta$ and $m, n \geq \max \left\{L, 100 d_{0}\right\}$ with $m n=A / a$ such that $h_{\theta}(20 m, n) \geq c$ and $v_{\theta}(m, 20 n) \geq c$, for some constant $c$ that is independent of $A$. Consider the image of Figure 6.1 (a) under a linear transformation mapping each 1-by-1 square to a $\theta$-aligned $m$-by- $n$ rectangle. Note that the resulting ellipse $E$ (the image of $C$ ) has both axes at least $L$ and has area $A$. Using Corollary 4.10 to allow for translation, each image rectangle has a black crossing in the relevant direction with probability at least some constant $c^{\prime}=\psi(c)>0$. Using Lemma 3.2 (in the form (14) ), it follows that $\mathbb{P}\left(O_{\mathrm{b}}(E)\right)$ is bounded away from zero.

For the reverse implication, we assume the large ellipses property and deduce the property $L R_{2}$. As shown in Figure 6.1(b), one can arrange 9 circles $C_{i}$ of radius 0.9 to 'cross' a 2 -by- 1 rectangle $R_{1}$ and a 1-by-2 rectangle $R_{2}$ in such a 
way that if $O_{\mathrm{b}}\left(C_{i}\right)$ holds for each $i$, then $H_{\mathrm{b}}\left(R_{1}\right)$ and $V_{\mathrm{b}}\left(R_{2}\right)$ hold. Moreover, this remains true if each circle is translated by a small distance (at most 0.01, say). Given an ellipse $E$ with both axes at least $\max \left\{100 d_{0}, L\right\}$ such that $\mathbb{P}\left(O_{\mathrm{b}}(E)\right) \geq c$, one can rotate the arrangement in Figure 6.1(b) and then scale it along the directions of the axes of $E$ so that each circle is mapped to a translate $E_{i}$ of $E$, and $R_{1}$ and $R_{2}$ are mapped to $\theta$-aligned $2 m$-by- $n$ and $m$-by$2 n$ rectangles $R_{1}^{\prime}$ and $R_{2}^{\prime}$, for some $\theta, m$ and $n$. Note that $m, n \geq L$, and $m n$ is an absolute constant times the area of $E$.

Moving each $E_{i}$ by a distance of at most $d_{0}$ (which corresponds to translating $C_{i}$ through a distance of at most 0.01), we may assume that $E_{i}=E+\ell_{i}$ for some $\ell_{i} \in \mathcal{L}$. Then $\mathbb{P}\left(O_{\mathrm{b}}\left(E_{i}\right)\right)=\mathbb{P}\left(O_{\mathrm{b}}(E)\right) \geq c$, and if all 9 upsets $O_{\mathrm{b}}\left(E_{i}\right)$ hold, then $H_{\mathrm{b}}\left(R_{1}^{\prime}\right)$ and $V_{\mathrm{b}}\left(R_{2}^{\prime}\right)$ hold. Using Lemma 3.2 thus gives a constant lower bound on $h_{\theta}(2 m, n)$ and $v_{\theta}(m, 2 n)$, establishing the property $L R_{2}$.

Most of the time, we work with the large rectangles property; the large ellipses property will be convenient to use in Section 8. The equivalence is also useful in that it leads to a quick proof that the large rectangles property is affine-invariant.

Lemma 6.6. Let $T$ be an invertible linear map from $\mathbb{R}^{2}$ to $\mathbb{R}^{2}$ and let $\mathcal{C}$ be a non-degenerate independent lattice colouring. If $\mathcal{C}$ has the large rectangles property, then so does $T(\mathcal{C})$.

Proof. There is a constant $a=a(T)>0$ such that if $E$ is an ellipse both of whose axes have length at least $L$ then $T(E)$ is an ellipse both of whose axes have length at least $a L$. (For example, note that $E$ contains a circle of radius $L$, and the image of this circle contains a circle of radius $a L$ for some $a>0$.) Hence the large ellipses property is invariant under T. Applying Lemma 6.5 twice, we see that the large rectangles property is too.

Our next lemma shows that after a linear transformation, a self-dual hyperlattice percolation model must be related to its dual by one of a small number of linear transformations. We consider both the self-dual case (for Theorem 2.1) and the approximately self-dual case (for Theorem 2.2).

Lemma 6.7. Let $\mathcal{H}(\mathbf{p})$ be an (approximately) self-dual hyperlattice percolation model. Then there is a map $S: \mathbb{R}^{2} \rightarrow \mathbb{R}^{2}$ giving an isomorphism between $\mathcal{H}(\mathbf{p})$ and a hyperlattice model equivalent to $\mathcal{H}^{*}\left(\mathbf{p}^{*}\right)$ such that $S$ can be written in the form $S(x)=T(x)+\Delta(x)$, where $T$ is linear and $|\Delta(x)|$ is bounded. Furthermore, after a linear change of coordinates (if necessary), we may assume that $T$ is either reflection in some line, or rotation through one of the angles $0, \pi / 2$, or $\pi$.

Proof. Suppose first that $\mathcal{H}(\mathbf{p})$ is self-dual. By the definition of self-duality for hyperlattices, there is a homeomorphism $S$ from $\mathbb{R}^{2}$ to $\mathbb{R}^{2}$ mapping $\mathcal{H}$ to its dual, and preserving the lattice structure. More precisely, there is a linear map $T$ with $T(\mathcal{L})=\mathcal{L}$ such that $S(x+\ell)=S(x)+T(\ell)$ whenever $\ell \in \mathcal{L}$. Since 
$\Delta=S-T$ is continuous and doubly periodic, it is bounded, giving the first statement.

Since $T(\mathcal{L})=\mathcal{L}$, the map $T$ preserves area, so $T \in G L_{2}\left(\mathbb{R}^{2}\right)$ with $\operatorname{det}(T)=$ \pm 1 . From standard results, $T$ is conjugate in $G L_{2}\left(\mathbb{R}^{2}\right)$ to a map $T^{\prime}$ that is either a rotation, a shear with matrix $\left(\begin{array}{ll}1 & \lambda \\ 0 & 1\end{array}\right)$, or a stretch with matrix $\left(\begin{array}{cc}\lambda & 0 \\ 0 & \pm 1 / \lambda\end{array}\right)$. Changing coordinates (or applying a linear transformation to $\mathcal{H}$ and its dual simultaneously), we may assume that $T=T^{\prime}$.

Now $S^{2}$ maps $\mathcal{H}$ into itself, and $S^{2}(x)=T^{2}(x)+\Delta_{2}(x)$ where $\Delta_{2}$ is bounded. If $T$ is a shear with $\lambda \neq 0$ or a stretch with $|\lambda| \neq 1$, then repeated application of $S^{2}$ shows the existence of arbitrarily long edges in $\mathcal{H}$, giving a contradiction. Thus $T$ is either a rotation or a reflection. In the former case, the fact that $T$ maps $\mathcal{L}$ into itself guarantees that the angle of rotation $\theta$ is a multiple of either $\pi / 3$ or $\pi / 2$. Replacing $S$ by the isomorphism $S^{3}$ from $\mathcal{H}(\mathbf{p})$ to its dual allows us to reduce the cases $\theta=k \pi / 3, k \in \mathbb{Z}$, to the cases $\theta=0$ or $\theta=\pi$, and the case $\theta=3 \pi / 2$ to the case $\theta=\pi / 2$.

The argument for the approximately self-dual case is similar, except that from the definition of approximate self-duality we may simply assume that $T$ is a rotation or a reflection.

For the rest of the section, in the light of Lemma 6.6. we assume as we may that $\mathcal{H}(\mathbf{p})$ and its dual are related as described in Lemma 6.7 In the case where the map $T$ is a reflection, we take it to be reflection in the $x$-axis, and call this the reflection case. The remaining cases are rotation cases; we write $\theta^{*}$ for the angle of rotation passing from $\mathcal{H}$ to $\mathcal{H}^{*}$, so $\theta^{*} \in\{0, \pi / 2, \pi\}$.

Let $\mathcal{C}^{*}$ be the negative of the colouring $\mathcal{C}$, defined simply by interchanging black and white (both in $\mathcal{H}$, and in the colours of the subfaces of the grey faces of $\mathcal{H})$. Even in the self-dual case, although $\mathcal{C}$ realizes $\mathcal{H}(\mathbf{p})$, which is selfdual via the map $S: \mathbb{R}^{2} \rightarrow \mathbb{R}^{2}$, we cannot assume that $\mathcal{C}$ is self-dual in the natural sense. For example, in the dual colouring to that shown in the centre of Figure 4.1, there are two black subfaces within the hexagon. This pattern may not occur in $\mathcal{C}$. However, writing $d_{0}$ for the constant given by Lemma 4.2, since $\mathcal{C}$ realizes $\mathcal{H}(\mathbf{p})$, there is a natural coupling of $\mathcal{C}$ and $\mathcal{H}(\mathbf{p})$ such that any black path $P$ in $\mathcal{C}$ is within Hausdorff distance $d_{0}$ of an open path in $\mathcal{H}(\mathbf{p})$, and vice versa. Applying this observation also to $\mathcal{C}^{*}$, which realizes $\mathcal{H}^{*}\left(\mathbf{p}^{*}\right)=S(\mathcal{H}(\mathbf{p}))$, it follows that we can couple $\mathcal{C}$ and $\mathcal{C}^{*}$ so that for any black path $P$ in $\mathcal{C}$ there is a black path in $\mathcal{C}^{*}$ within distance $O(1)$ of $S(P)$, and vice versa. Recalling that black in $\mathcal{C}^{*}$ simply means white in $\mathcal{C}$, and using Lemma 6.7 this has the following consequence. Here, $\varphi$ is the 'probability scaling function' defined before Lemma 4.8 .

Lemma 6.8. Let $\mathcal{C}$ be a non-degenerate independent lattice colouring realizing an (approximately) self-dual hyperlattice percolation model $\mathcal{H}(\mathbf{p})$, and let $T$ be the linear map given by Lemma 6.7. Then there are constants $L$ and $C$ such that, for any angle $\theta$ and any $\theta$-aligned $m$-by-n rectangle $R$ with $m, n \geq L$, we have

$$
\varphi\left(\mathbb{P}\left(H_{\mathrm{b}}(R)\right)\right)-\varphi\left(\mathbb{P}\left(H_{\mathrm{w}}(T(R))\right)\right) \in[-C, C]
$$


Proof. We write out only the self-dual case; since we in any case allow some 'elbow room' when passing from $\mathcal{H}(\mathbf{p})$ to its dual, there are no additional difficulties in the approximately self-dual case.

Let $L=100 d_{0}+10 D$, where $D=\sup \{|S(x)-T(x)|\}$, which is finite by Lemma 6.7 Couple $\mathcal{C}$ and $\mathcal{C}_{1}=\mathcal{C}^{*}$ as above. Let $\mathcal{C}_{2}$ be the colouring obtained from $\mathcal{C}_{1}$ by interchanging white and black, so $\mathcal{C}_{2}$ has the same distribution as $\mathcal{C}$.

Let $R$ be a rectangle as described, and let $R^{+}$be obtained by moving the vertical sides of $R$ outwards by a distance $D$ and the horizontal sides inwards by the same distance. Suppose $R^{+}$has a black horizontal crossing in $\mathcal{C}$. Then from the remarks before the lemma, there is a black path in $\mathcal{C}_{1}$ close to $S(P)$ and hence to $T(P)$, and thus a white path in $\mathcal{C}_{2}$ close to $T(P)$. But any such path crosses $T(R)$. Hence

$$
\mathbb{P}_{\mathcal{C}}\left(H_{\mathrm{w}}(T(R))\right)=\mathbb{P}_{\mathcal{C}_{2}}\left(H_{\mathrm{w}}(T(R))\right) \geq \mathbb{P}_{\mathcal{C}}\left(H_{\mathrm{b}}\left(R^{+}\right)\right) .
$$

Writing $\mathbb{P}$ for $\mathbb{P}_{\mathcal{C}}$ as usual, and using Lemma 4.8, this gives $\varphi\left(\mathbb{P}\left(H_{\mathrm{w}}(T(R))\right)\right) \geq$ $\varphi\left(\mathbb{P}\left(H_{\mathrm{b}}(R)\right)\right)-O(1)$. The reverse inequality is proved similarly.

In what follows we assume throughout that our 'large' length $L$ is at least $100 d_{0}$, and is such that Lemma 6.8 applies. Note that rectangles with dimensions at least $L$ are 'large' in the terminology of the previous sections.

Lemma 6.9. Let $\mathcal{C}$ be a non-degenerate independent lattice colouring realizing an (approximately) self-dual hyperlattice percolation model $\mathcal{H}(\mathbf{p})$. For any $L$ there are constants $A_{0}=A_{0}(\mathcal{C}, L)$ and $C=C(\mathcal{C}, L)$ such that for any $A \geq A_{0}$, and any $\theta$ in the rotation case or $\theta=0$ in the reflection case, we may find $m$, $n \geq L$ with $m n=A$ such that $\varphi\left(h_{\theta}(m, n)\right), \varphi\left(v_{\theta}(m, n)\right) \in[-C, C]$.

In other words, both the horizontal and vertical crossing probabilities for some rectangle of the given area and orientation are bounded away from 0 and 1.

Proof. Let us write $h_{\theta}^{*}(m, n)$ and $v_{\theta}^{*}(m, n)$ for the probabilities that an $m$-by-n $\theta$-aligned rectangle has a white horizontal crossing or a white vertical crossing, respectively.

Consider first the rotation case with $\theta^{*}=\pi / 2$. In this case we simply set $A_{0}=L^{2}$, and choose $m=n=\sqrt{A}$. Let $R$ be the $m$-by- $m \theta$-aligned square centred on the origin. Note that $T(R)$ is the same rectangle $R$, but viewed as $(\theta+\pi / 2)$-aligned, so $v_{\theta}^{*}(m, m)=\mathbb{P}\left(H_{\mathrm{w}}(T(R))\right)$. Hence Lemma 6.8 gives $\varphi\left(h_{\theta}(m, m)\right)-\varphi\left(v_{\theta}^{*}(m, m)\right)=O(1)$. But by Lemma 4.4. we have $h_{\theta}(m, m)+$ $v_{\theta}^{*}(m, m)=1$, so $\varphi\left(v_{\theta}^{*}(m, m)\right)=-\varphi\left(h_{\theta}(m, m)\right)$. It follows that $\varphi\left(h_{\theta}(m, m)\right)=$ $O(1)$. Similarly, $\varphi\left(v_{\theta}(m, m)\right)=O(1)$.

For the remaining cases, for $A$ large enough, Lemma 4.12 gives us $m, n \geq L$ with $m n=A$ and $\varphi\left(h_{\theta}(m, n)\right) \in[-4,4]$. This time (noting that $\theta=0$ in the reflection case), the map $T$ maps our $\theta$-aligned $m$-by- $n$ rectangle into itself, and Lemma 6.8 gives $\varphi\left(h_{\theta}(m, n)\right)-\varphi\left(h_{\theta}^{*}(m, n)\right)=O(1)$. Hence $\varphi\left(v_{\theta}(m, n)\right)=$ $-\varphi\left(h_{\theta}^{*}(m, n)\right)=\varphi\left(h_{\theta}(m, n)\right)+O(1)=O(1)$. 
We are now ready to prove Theorem 6.3.

Proof of Theorem 6.3. We assume as we may that $L \geq 100 d_{0}$. Throughout we fix an arbitrary $A \geq 10^{6} A_{0}(\mathcal{C}, L)$, where $A_{0}(\mathcal{C}, L)$ is the constant in Lemma 6.9 All constants $c$ or $c_{i}$ below will depend only on $\mathcal{C}$, not on $A$ or $L$. We shall show that for some $\theta, a$ and $b$ with $a b=10 A$ we have

$$
h_{\theta}(10 a, b), v_{\theta}(a, 10 b) \geq c,
$$

where $c>0$ does not depend on $A$. This will establish that $\mathcal{C}$ has the large rectangles property $L R_{10}$.

Fix an orientation $\theta$, with $\theta=0$ in the reflection case, and $\theta$ arbitrary otherwise. Suppressing the dependence on $\theta$ in the notation, by Lemma 6.9 there are $m, n \geq L$ with $m n=A$ such that

$$
h(m, n), v(m, n) \geq c_{0},
$$

where $c_{0}>0$ is a constant depending only on $\mathcal{C}$.

Suppose for the moment that $h(100 m, n) \geq c_{1}$, where $c_{1}$ is a positive constant not depending on $A$. Set $m^{\prime}=10 m$ and $n^{\prime}=n$, and consider an $m^{\prime}$-by- $n^{\prime}$ rectangle $R$ and the $m^{\prime} / 10$-by- $n^{\prime}$ rectangle $R^{\prime}$ inside it. Note that $h(R)=h(10 m, n) \geq h(100 m, n) \geq c_{1}$. Also, since $R^{\prime}$ is $m$-by- $n$, we have $v\left(R^{\prime}\right) \geq c_{0}$. We may thus apply Theorem 5.12 with $m$ and $n$ replaced by $m^{\prime}$ and $n^{\prime}$, and $\alpha=\beta_{1}=1 / 10, \beta_{2}=1$, and $\eta=1 / 2$. Any black vertical crossing of $R^{\prime}$ satisfies the conditions for the event $E_{\mathrm{v}}$ considered in Theorem 5.12 simply because, seen with respect to the $m^{\prime}$-by- $n^{\prime}$ grid, $R^{\prime}$ is much taller than wide. Theorem 5.12 thus gives $v\left(m^{\prime}, 100 n^{\prime}\right) \geq c$, for some constant $c>0$. But then $h\left(10 m^{\prime}, n^{\prime}\right)=h(100 m, n)$ and $v\left(m^{\prime}, 10 n^{\prime}\right) \geq v\left(m^{\prime}, 100 n^{\prime}\right)$ are both at least $\min \left\{c_{0}, c\right\}$, establishing (17) with $a=m^{\prime}=10 m$ and $b=n^{\prime}=n$.

A similar argument (using a 'rotated' version of Theorem 5.12) shows that if $v(m, 100 n) \geq c_{1}$, then (17) holds with $a=m$ and $b=10 n$. Thus, in what follows, it suffices to show that one of $h(100 m, n)$ and $v(m, 100 n)$ is not too small.

Applying Lemma 6.9 again we find $m_{1}, n_{1} \geq L$ with $m_{1} n_{1}=m n / 400=$ $A / 400$ such that

$$
h\left(m_{1}, n_{1}\right), v\left(m_{1}, n_{1}\right) \geq c_{0} .
$$

Let $R_{1}$ be the $m_{1}$-by- $n_{1}$ rectangle centred on the origin, so $\mathbb{P}\left(H_{\mathrm{b}}\left(R_{1}\right)\right) \geq c_{0}$ and $\mathbb{P}\left(V_{\mathrm{b}}\left(R_{1}\right)\right) \geq c_{0}$.

Fix a small constant $\delta>0$; for example, $\delta=1 / 1000$ will do. Suppose first that $n_{1} / n \geq(1+\delta) m_{1} / m$, say. Then on the $m$-by- $n$ scale, the rectangle $R_{1}$ is significantly taller than wide, and has area $1 / 400$. After truncating $R_{1}$ vertically if necessary (after which we still have $\mathbb{P}\left(V_{\mathrm{b}}\left(R_{1}\right)\right) \geq c_{0}$ ), we may apply Theorem 5.12 with $R$ an $m$-by- $n$ rectangle, with $\beta_{1}=m_{1} / m \leq 1 / 20, \alpha=\beta_{2}=$ $\min \left\{n_{1} / n, 1 / 3\right\} \geq(1+\delta) m_{1} / m$, and $\eta=\delta / 2$. A black vertical crossing of $R_{1}$ once again ensures that $E_{\mathrm{v}}$ holds, and it follows that $v(m, 100 n) \geq c$ for some constant $c>0$. As noted above, we are done in this case. 
A similar argument applies if $m_{1} / m \geq(1+\delta) n_{1} / n$, so we may assume that $(1-\delta) / 20 \leq m_{1} / m, n_{1} / n \leq(1+\delta) / 20$.

As before, let $F$ be the function given by Lemma 3.2 applied to the product of partially ordered sets corresponding to the random partitions induced by each edge $e$ of $\mathcal{H}$. Recalling that $h\left(m_{1}, n_{1}\right), v\left(m_{1}, n_{1}\right) \geq c_{0}$, set $c_{2}=F\left(\psi\left(c_{0}\right), \psi\left(c_{0}\right)\right)$, where $\psi$ is the function appearing in Corollary 4.10. For any rectangle $R$, let $X(R)$ denote the event $H_{\mathrm{b}}(R) \cap V_{\mathrm{b}}(R)$ that $R$ has both horizontal and vertical black crossings. For any $m_{1}$-by- $n_{1}$ rectangle $R$, we have $\mathbb{P}\left(H_{\mathrm{b}}(R)\right)$, $\mathbb{P}\left(V_{\mathrm{b}}(R)\right) \geq \psi\left(c_{0}\right)$ by Corollary 4.10 , and thus

$$
\mathbb{P}(X(R)) \geq c_{2} .
$$

Set $c_{3}=F\left(c_{2} / 4, c_{2} / 4\right)<c_{2} / 4$, and $c_{4}=F\left(c_{3}, c_{3} / 2\right)<c_{3} / 2$.

Let us say that a rectangle $R$ is useful if it contains two black paths $P_{1}$ and $P_{2}$ such that, after scaling by dividing all $x$-coordinates by $m$ and all $y$-coordinates by $n$, the paths satisfy the assumptions of Lemma 5.17. In other words, for each $i$, the vector $v_{i}$ from the start of $P_{i}$ to the end has (after rescaling) length at least $\delta$, and the angle between $v_{1}$ and $v_{2}$ is at least $\delta$. We write $U(R)$ for the event that $R$ is useful. If there is any rectangle $R$ with width at most $m / 10$ and height at most $n / 10$ for which $\mathbb{P}(U(R)) \geq c_{4}$ then, recalling (18), Theorem 5.18 gives $v(m, 100 n) \geq c$ for some $c>0$. Hence we may assume that

$$
\mathbb{P}(U(R))<c_{4}<c_{3} / 2<c_{2} / 8
$$

for any rectangle with these dimensions. Note that $1.5 m_{1} \leq m / 10$ and $1.5 n_{1} \leq$ $n / 10$, so the rectangle $R_{1}$ defined earlier satisfies the size restriction above with room to spare.

Suppose that some $m_{1}$-by- $n_{1}$ rectangle $R$ is coloured in such a way that $X(R) \backslash U(R)$ holds. Then $R$ has a black horizontal crossing $P_{1}$ and a black vertical crossing $P_{2}$. The angle (after scaling) between these crossings must be very close to 0 , so it follows that either both $P_{1}$ and $P_{2}$ cross $R$ from near the top left to near the bottom right, or both cross from near the bottom left to near the top right. Suppose the former holds. Then, in addition, every black horizontal or vertical crossing stays (after the usual scaling) within distance $5 \delta$ of the diagonal; otherwise, such a crossing can be split into two parts $(A B$ and $B C$ in the figure) with an angle of at least $\delta$ between them, so $U(R)$ holds; see Figure 6.2.

Let $D_{1}=D_{1}(R)$ and $D_{2}=D_{2}(R)$ denote the strips of width (after rescaling as above) $5 \delta$ about the two diagonals of $R$. Let $X_{i}(R)$ denote the event that $D_{i}(R)$ contains both horizontal and vertical crossings of $R$; then $X(R) \backslash U(R) \subset$ $X_{1}(R) \cup X_{2}(R)$. Hence, for any $m_{1}$-by- $n_{1}$ rectangle $R$, for some $i$ we have $\mathbb{P}\left(X_{i}(R)\right) \geq(\mathbb{P}(X(R))-\mathbb{P}(U(R))) / 2 \geq c_{2} / 4$, using (19) and (20). We say that $R$ is of type $i$ if $\mathbb{P}\left(X_{i}(R)\right) \geq c_{2} / 4$, so any $R$ is of type 1 or type 2 . Furthermore, no $R$ can be of both types: otherwise, using Lemma 3.2 again, we have $\mathbb{P}(U(R)) \geq$ $\mathbb{P}\left(X_{1}(R) \cap X_{2}(R)\right) \geq F\left(c_{2} / 4, c_{2} / 4\right)=c_{3}$, contradicting (20).

At this point we consider two separate cases. 

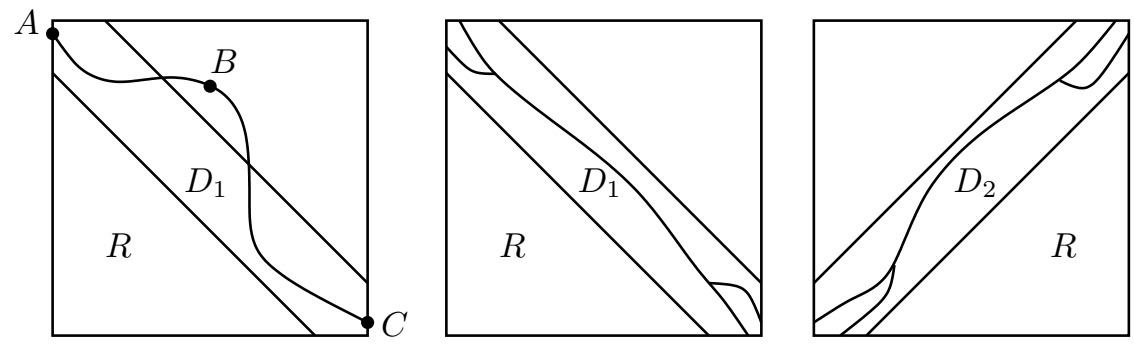

Figure 6.2: Various possible configurations in an $m_{1}$-by- $n_{1}$ rectangle $R$, rescaled by dividing $x$-coordinates by $m$ and $y$-coordinates by $n$. Note that the width and height of $R$ are then between $(1-\delta) / 20$ and $(1+\delta) / 20$. The strips $D_{1}$ and $D_{2}$ have width $10 \delta$. The second and third figures illustrate $X_{1}(R)$ and $X_{2}(R)$.

1. Reflection case. Let $R_{1}$ and $R_{2}$ be two $m_{1}$-by- $n_{1}$ rectangles with $R_{2}$ obtained by translating $R_{1}$ vertically through a distance $10 \delta n \leq n_{1} / 10$. Recalling that our orientation $\theta$ is the standard orientation $\theta=0$ in this case, we may choose $R_{1}$ and $R_{2}$ so that the rectangle $R_{1} \cup R_{2}$ is centred at the origin; in particular, the $x$-axis is an axis of symmetry of $R_{1} \cup R_{2}$.

Suppose first that $R_{1}$ and $R_{2}$ are of opposite types, say with $R_{i}$ of type $i$. Then $\mathbb{P}\left(U\left(R_{1} \cup R_{2}\right)\right) \geq \mathbb{P}\left(X_{1}\left(R_{1}\right) \cap X_{2}\left(R_{2}\right)\right) \geq F\left(c_{2} / 4, c_{2} / 4\right)=c_{3}$, contradicting (20). It follows that $R_{1}$ and $R_{2}$ must be of the same type, say type 1 .

Let $E$ be the event $X_{1}\left(R_{1}\right) \cap X_{1}\left(R_{2}\right) \backslash U\left(R_{1} \cup R_{2}\right)$, so $\mathbb{P}(E) \geq F\left(c_{2} / 4, c_{2} / 4\right)-$ $c_{3} / 2=c_{3} / 2$ by (20). If $E$ holds, then $R_{1}$ contains a black horizontal crossing $P_{1}$ in the strip $D_{1}=D_{1}\left(R_{1}\right)$, and $R_{2}$ contains a black horizontal crossing $P_{2}$ in the strip $D_{1}^{\prime}=D_{1}\left(R_{2}\right)$. It is easy to check that if $P_{1}$ and $P_{2}$ are joined by a black path in $R_{1} \cup R_{2}$, then $U\left(R_{1} \cup R_{2}\right)$ holds. (For example, since we also have vertical crossings of $R_{1}$ and $R_{2}$, we obtain a vertical crossing of $R_{1} \cup R_{2}$, whose direction is necessarily more than an angle $\delta$ away from that of $P_{1}$ or $P_{2}$.) Hence, whenever $E$ holds, so does the event $F$, that $D_{1} \cup D_{1}^{\prime}$ contains a white horizontal crossing of $R_{1} \cup R_{2}$. Thus, $\mathbb{P}(F) \geq c_{3} / 2$.

We now apply self-duality as in the proof of Lemma 6.8 recalling that now $T$ is reflection in the $x$-axis, an axis of symmetry of $R_{1} \cup R_{2}$. Let $\tilde{F}$ be the image of $F$ under the symmetry transformation. Then $\mathbb{P}(\tilde{F})=\mathbb{P}(F)$. Also, if $\tilde{F}$ holds then there is a black path in $R_{1} \cup R_{2}$ that almost crosses $R_{1} \cup R_{2}$ horizontally, and lies within or close to the mirror image of $D_{1} \cup D_{1}^{\prime}$. Since $\tilde{F}$ is an upset, by Lemma 3.2 we have $\mathbb{P}\left(X_{1}\left(R_{1}\right) \cap X_{2}\left(R_{2}\right) \cap \tilde{F}\right) \geq F\left(c_{3}, c_{3} / 2\right)=c_{4}$. But whenever this event holds, $R_{1} \cup R_{2}$ is clearly useful: the relevant crossings must in fact meet, but we do not even need this, simply the observation that $R_{1} \cup R_{2}$ then contains two longish black paths that, after the usual rescaling, are at almost 90 degrees to each other. Thus $\mathbb{P}\left(U\left(R_{1} \cup R_{2}\right)\right) \geq c_{4}$, contradicting (20). This contradiction completes the proof in the reflection case.

2. Rotation case. In this case, Lemma 6.9 applies regardless of the orien$\operatorname{tation} \theta$. The idea is to observe that the argument above shows that for each $\theta$, the $\theta$-aligned $m_{1}$-by- $n_{1}$ rectangle $R_{1}$ centred on the origin is either of type 1 
or of type 2. As we rotate, we can assume (as we will shortly show) that $R_{1}$ varies continuously. Thus it should not jump from type 1 to type 2 . But after rotating by 90 degrees, we return to the same rectangle viewed with a different orientation. Changing orientation in this way interchanges types 1 and 2 .

To make this precise, first note that the argument above establishes the following. Let $C$ be the constant in Lemma 6.9, let $\theta$ be any orientation, and let $m_{1}$ and $n_{1}$ satisfy $m_{1} n_{1}=A / 400$ and $m_{1}, n_{1} \geq L$. If $R$ is the $\theta$-aligned $m_{1}$-by- $n_{1}$ rectangle centred on the origin, and $\varphi\left(h_{\theta}\left(m_{1}, n_{1}\right)\right), \varphi\left(v_{\theta}\left(m_{1}, n_{1}\right)\right) \in$ [ $-C-8, C+8]$, then $R$ is either of type 1 or of type 2 (and not both, although we shall not use this). To see this, simply use the rectangle $R$ under consideration as $R_{1}$ in the argument above: so far we selected an arbitrary rectangle with the properties described by Lemma 6.9 now we choose a specific one with the same properties, except that we have replaced $C$ by $C+8$, which makes no difference.

Pick an integer $k \geq A / d_{0}$, and let $\varepsilon=(\pi / 2) / k$. We claim that we can construct a finite sequence $R_{0}, R_{1}, \ldots, R_{N}$ of rectangles, all centred on the origin, where $R_{i}$ is $\theta_{i}$-aligned and satisfies the conditions above, with $\theta_{0}=0, \theta_{N}=\pi / 2$ and $R_{0}$ and $R_{N}$ the same geometric rectangle, such that for $i=0,1, \ldots, N-1$, either $R_{i}$ and $R_{i+1}$ have the same dimensions and $\theta_{i+1}=\theta_{i}+\varepsilon$, or $R_{i}$ and $R_{i+1}$ have the same orientation, and their corresponding dimensions differ by at most one.

To establish the claim, simply start with $\theta_{0}=0$ and $R_{0}$ of the dimensions given by Lemma 6.9. At each stage, if $R_{i}$ satisfies $\varphi\left(h\left(R_{i}\right)\right), \varphi\left(v\left(R_{i}\right)\right) \in[-C, C]$, then rotate it through an angle $\varepsilon$ to obtain $R_{i+1}$. Since the largest dimension of $R$ is at most $(A / 400) /\left(100 d_{0}\right) \leq k / 10$, Corollary 4.11 ensures that $R_{i+1}$ has the required properties. Otherwise, by assumption we have $\varphi\left(h\left(R_{i}\right)\right)$, $\varphi\left(v\left(R_{i}\right)\right) \in[-C-8, C+8]$. Applying Lemma [6.9, pick a rectangle $R$ with the same orientation and area for which $\varphi(h(R)), \varphi(v(R)) \in[-C, C]$. Since $R_{i}$ and $R$ have the same orientation, we may pass gradually from one to the other with $h(\cdot)$ increasing and $v(\cdot)$ decreasing or vice versa; the intermediate rectangles thus all satisfy our requirements. Once we reach $R$, we are ready for the next rotation step.

Since the type of $R_{N}$ is the opposite of that of $R_{0}$, there is some $i$ such that $R_{i}$ and $R_{i+1}$ have different types; suppose that $R_{i}$ is of type 1 and $R_{i+1}$ of type 2 . Then by Lemma 3.2 we have $\mathbb{P}\left(X_{1}\left(R_{i}\right) \cap X_{2}\left(R_{i+1}\right)\right) \geq c_{3}$. Although the dimensions of our rectangles may change radically as we rotate them, they cannot do so in one step. In particular, after the rescaling above applied to $R_{i}$, the paths witnessing $X_{1}\left(R_{i}\right)$ and $X_{2}\left(R_{i+1}\right)$ are close to orthogonal. It follows that if $X_{1}\left(R_{i}\right) \cap X_{2}\left(R_{i+1}\right)$ holds, then $R_{i}$ is useful, so $\mathbb{P}\left(U\left(R_{i}\right)\right) \geq c_{3}$, contradicting (20) above.

\section{From rectangle crossings to percolation}

In this section we shall deduce Theorems 2.1 and 2.2 from Theorem 6.3, this turns out to be relatively straightforward, adapting the very simple argument for bond percolation on $\mathbb{Z}^{2}$ described in [4. First, we get one technical detail 
out of the way.

Recall that we can represent any hyperlattice percolation model $\mathcal{H}(\mathbf{p})$ by an independent lattice colouring $\mathcal{C}$; our results in the previous section apply only to malleable $\mathcal{C}$. In the bulk of this section we shall prove the following variant of Theorem 2.1, differing only in the additional assumption of malleability.

Theorem 7.1. Let $\mathcal{H}(\mathbf{p})$ be a malleable (approximately) self-dual hyperlattice percolation model. Then for any $\mathbf{q} \succ \mathbf{p}$ the model $\mathcal{H}(\mathbf{q})$ percolates, and for any $\mathbf{q} \prec \mathbf{p}$ the model $\mathcal{H}(\mathbf{q})$ exhibits exponential decay.

Before proving this result, we note that Theorem 2.1 follows.

Proof of Theorem 2.1. Let $\mathcal{H}(\mathbf{p})$ be self-dual, and let $S$ be an isomorphism from $\mathcal{H}$ to $\mathcal{H}^{*}$ witnessing this. For this $S$, the self-duality condition reduces to a set of equations equating certain entries $p_{i, \pi}$ of $\mathbf{p}$. In particular, any entry $p_{i, \pi}$ where $\pi$ is a partition into singletons is equated with some $p_{j, \pi^{\prime}}$, where $\pi^{\prime}$ is a partition into one part. Recall that we call entries of these two types bottom and top entries, respectively.

If $\mathbf{q} \succ \mathbf{p}$, then by definition of our partial order, each bottom entry of $\mathbf{p}$ is non-zero. Hence by self-duality each top entry is non-zero. Since corresponding top or bottom entries of $\mathbf{p}$ and $\mathbf{q}$ cannot be equal, it follows that we can adjust $\mathbf{p}$ slightly to find some $\mathbf{p}^{\prime}$ all of whose entries are strictly positive such that $\mathcal{H}\left(\mathbf{p}^{\prime}\right)$ is self-dual, with $\mathbf{p}^{\prime} \prec \mathbf{q}$. Since $\mathbf{p}^{\prime}$ is malleable, Theorem 7.1 implies that $\mathcal{H}(\mathbf{q})$ percolates, as required.

The argument that any $\mathbf{q} \prec \mathbf{p}$ exhibits exponential decay proceeds similarly.

The argument above shows that in proving Theorem 2.1, we may impose the condition of malleability (which we need in the proof) without loss of generality, so we do not need to assume malleability in the statement of the theorem. Unfortunately, there does not seem to be an obvious analogous argument in the approximately self-dual case: it is not clear how to adjust the probabilities slightly while preserving approximate self-duality. For this reason we simply impose malleability as a condition in Theorem 2.2. so Theorem 2.2 trivially follows from Theorem 7.1 .

To deduce Theorem 7.1 from Theorem 6.3 we shall use an analogue for posets of the well-known Friedgut-Kalai sharp-threshold result for symmetric events, Theorem 2.1 of [12, which is itself a consequence of a result of Kahn, Kalai and Linial [15] (see also 8]) concerning the influences of coordinates in a product space. This sharp-threshold result has been applied in many contexts - it was first used to prove criticality (for random Voronoi percolation) in [3]; we shall use the same technique here (see also [4).

Let $\mathcal{P}$ be a finite poset. Given two probability measures $\mathbb{P}_{0}$ and $\mathbb{P}_{1}$ on $\mathcal{P}$, recall that $\mathbb{P}_{1}$ strictly dominates $\mathbb{P}_{0}$, written $\mathbb{P}_{1} \succ \mathbb{P}_{0}$, if $\mathbb{P}_{1}(\mathcal{U})>\mathbb{P}_{0}(\mathcal{U})$ for every upset $\mathcal{U} \subset \mathcal{P}$, except the trivial upsets $\mathcal{U}=\emptyset, \mathcal{P}$.

As usual, given a (po)set $\mathcal{P}$ and a subset $\mathcal{A}$ of $\mathcal{P}^{n}$, a coordinate $i$ is pivotal for $\mathcal{A}$ in a configuration $\omega \in \mathcal{P}^{n}$ if changing the $i$ th coordinate of $\omega$ can affect 
whether $\omega \in \mathcal{A}$. Let $\mathcal{A}_{i}(\omega) \subset \mathcal{P}$ denote the set of values that, when substituted for the $i$ th coordinate of $\omega$, give some $\omega^{\prime} \in \mathcal{A}$. Thus $i$ is pivotal for $\mathcal{A}$ in $\omega$ if and only if $\emptyset \neq \mathcal{A}_{i}(\omega) \neq \mathcal{P}$. If $\mathcal{A}$ is an upset, then $\mathcal{A}_{i}(\omega)$ is an upset.

Given $\mathbb{P}_{0} \prec \mathbb{P}_{1}$, for $0<h<1$ define $\mathbb{P}_{h}$ by linear interpolation: $\mathbb{P}_{h}(x)=$ $h \mathbb{P}_{1}(x)+(1-h) \mathbb{P}_{0}(x)$ for all $x \in \mathcal{P}$. Let $c_{0}=c_{0}\left(\mathbb{P}_{0}, \mathbb{P}_{1}\right)$ be the minimum of $\mathbb{P}_{1}(\mathcal{U})-\mathbb{P}_{0}(\mathcal{U})$ over all non-trivial upsets in $\mathcal{P}$, so $c_{0}>0$ by assumption. Then, for any non-trivial upset $\mathcal{U}$, we have $\frac{\mathrm{d}}{\mathrm{d} h} \mathbb{P}_{h}(\mathcal{U}) \geq c_{0}$. Considering partial derivatives in a product with different values for $h$ in each coordinate, one obtains an analogue of the Margulis-Russo formula [20, 23]: if $\mathcal{A} \subset \mathcal{P}^{n}$ is an upset, then with $c_{0}=c\left(\mathbb{P}_{0}, \mathbb{P}_{1}\right)>0$ as above, we have

$$
\frac{\mathrm{d}}{\mathrm{d} h} \mathbb{P}_{h}^{n}(\mathcal{A}) \geq c_{0} \mathbb{E}_{h} N
$$

where $\mathbb{E}_{h}$ denotes expectation with respect to the product measure $\mathbb{P}_{h}^{n}$, and $N=$ $N(\omega)$ is the number of pivotal coordinates for $\mathcal{A}$ in the random configuration $\omega$.

Bourgain, Kahn, Kalai, Katznelson and Linial [8] showed that if $X$ is any probability space, and $\mathcal{A}$ is a subset of $X^{n}$, then then there is some coordinate $i$ such that the probability that $i$ is pivotal for $\mathcal{A}$ is at least $c t(1-t) \log n / n$, where $c>0$ is an absolute constant, and $t$ is the probability of $\mathcal{A}$. As usual, we say that $\mathcal{A}$ is symmetric if there is a permutation group acting transitively on $\{1,2, \ldots, n\}$ whose induced action on $X^{n}$ preserves $\mathcal{A}$. If $\mathcal{A}$ is symmetric, each coordinate has the same probability of being pivotal, so the expected number of pivotal elements is at least $c t(1-t) \log n$. Using (21) in place of the usual Margulis-Russo formula, one then obtains the following result; we omit the simple calculation, noting that one may take $c_{1}\left(\mathbb{P}_{0}, \mathbb{P}_{1}\right)=c c_{0}\left(\mathbb{P}_{0}, \mathbb{P}_{1}\right) / 2$.

Theorem 7.2. Let $\mathbb{P}_{0}$ and $\mathbb{P}_{1}$ be probability measures on a poset $\mathcal{P}$ with $\mathbb{P}_{0} \prec \mathbb{P}_{1}$. There is a constant $c_{1}\left(\mathbb{P}_{0}, \mathbb{P}_{1}\right)>0$ with the following property. Let $0<\varepsilon<1 / 2$, and let $\mathcal{A}$ be a symmetric, increasing event in a power $\mathcal{P}^{n}$ of $\mathcal{P}$ with $\mathbb{P}_{0}^{n}(\mathcal{A})>\varepsilon$. If

$$
c_{1}\left(\mathbb{P}_{0}, \mathbb{P}_{1}\right) \log n \geq \log (1 / \varepsilon),
$$

then $\mathbb{P}_{1}^{n}(\mathcal{A})>1-\varepsilon$.

Using Theorem 7.2 in place of the Friedgut-Kalai result, it is very simple to adapt (one of) the simple arguments given in 4 to deduce Theorem 7.1 from Theorem 6.3.

Proof of Theorem 7.1. Let $\mathcal{H}(\mathbf{p})$ be a malleable (approximately) self-dual hyperlattice percolation model, with $\mathcal{L}$ the corresponding lattice of translational symmetries, and let $\mathbf{q} \succ \mathbf{p}$. Note that since $\mathcal{H}(\mathbf{p})$ is non-degenerate, so is $\mathcal{H}\left(\mathbf{q}^{\prime}\right)$, where $\mathbf{q}^{\prime}=(\mathbf{p}+\mathbf{q}) / 2$. If some bottom entries in $\mathbf{q}$ are zero, we replace $\mathbf{q}$ by $\mathbf{q}^{\prime}$ in what follows: since $\mathbf{q} \succ \mathbf{q}^{\prime} \succ \mathbf{p}$, it suffices to prove percolation in $\mathcal{H}\left(\mathbf{q}^{\prime}\right)$. Thus we may assume without loss of generality that $\mathcal{H}(\mathbf{q})$ is non-degenerate.

As usual, we wish to work with crossings of rectangles, so it is more convenient to work with independent lattice colourings.

By Lemma 5.10 there is a malleable independent lattice colouring $\mathcal{C}_{0}$ realizing $\mathcal{H}(\mathbf{p})$. As in Section 4 (before Lemma 4.2), we regard the state space $\Omega$ 
underlying the random colouring $\mathcal{C}_{0}$ as a product of one poset $\mathcal{P}_{F}$ for each grey face $F$ of $\mathcal{H}$ : in the partial order, we have $c_{1} \preccurlyeq c_{2}$ if every subface that is black in $c_{1}$ is black in $c_{2}$. Picking a finite set $F_{1}, \ldots, F_{k}$ of faces representing the orbits of $E(\mathcal{H})$ (the set of grey faces) under the action of $\mathcal{L}$, from lattice invariance we may regard $\Omega$ as a countable power of the poset $\mathcal{P}=\mathcal{P}_{F_{1}} \times \cdots \times \mathcal{P}_{F_{k}}$. (As usual, the events we consider in the following arguments will be defined in terms of finite regions of the plane, and so can be viewed as events in a finite power of $\mathcal{P}$.)

From independence, the probability measure associated to $\mathcal{C}_{0}$ is a power of a probability measure $\mathbb{P}_{0}$ on $\mathcal{P}$. Furthermore, since $\mathbf{p} \prec \mathbf{q}$, we may choose another measure $\mathbb{P}_{1}$ on $\mathcal{P}$ with $\mathbb{P}_{0} \prec \mathbb{P}_{1}$ such that the corresponding independent lattice colouring $\mathcal{C}_{1}$ realizes $\mathcal{H}(\mathbf{q})$. We may and shall assume that $\mathcal{C}_{1}$ is non-degenerate.

Let $\delta(\mathcal{P}, \mathbb{P}, k, \varepsilon)$ be the function appearing in Corollary 3.3 (our version of the square-root trick), and set

$$
\delta=\delta\left(\mathcal{P}, \mathbb{P}_{1}, 100,0.01\right)>0 .
$$

Let $c>0$ be the constant in the $L R_{10}$ property of $\mathcal{C}_{0}$; such a constant exists by Theorem 6.3. Let $\varepsilon>0$ be the minimum of $c$ and $\delta$, and choose $N$ such that

$$
c_{1}\left(\mathbb{P}_{0}, \mathbb{P}_{1}\right) \log N \geq \log (1 / \varepsilon),
$$

where $c_{1}\left(\mathbb{P}_{0}, \mathbb{P}_{1}\right)$ is defined as in Theorem 7.2 Let $D=F_{1} \cup \cdots \cup F_{k}$, and choose $L_{0}$ so that $L_{0}^{2} \geq N$ area $(D)$, so any region of area at least $L_{0}^{2}$ meets at least $N$ translates of $D$ by elements of $\mathcal{L}$.

Let $d_{1}$ be the maximum of the quantity $d_{0}$ appearing in Lemma 4.2 and $\operatorname{diam}(D)$, the diameter of $D$. Since $\mathcal{C}_{0}$ has the large rectangles property $L R_{10}$, we can find an angle $\theta$ and $m, n \geq \max \left\{L_{0}, 100 d_{1}\right\}$ such that $h_{\theta}(10 m, n) \geq c$ and $v_{\theta}(m, 10 n) \geq c$. In other words, there are orthogonal vectors $v_{1}$ and $v_{2}$ (obtained by rotating $(m, 0)$ and $(0, n)$ through an angle $\theta)$ with the following property: the rectangles $R_{1}$ with corners $\pm 5 v_{1} \pm v_{2} / 2$ and $R_{2}$ with corners $\pm v_{1} / 2 \pm 5 v_{2}$ are such that the probability that $R_{i}$ has a 'long' (parallel to the $10 v_{i}$ side) black crossing in $\mathcal{C}_{0}$ is at least $c$.

By Lemma 4.2. every point of $\mathbb{R}^{2}$ is within distance $d_{1}$ of some point of $\mathcal{L}$, so we may find $\ell_{1}, \ell_{2} \in \mathcal{L}$ within distance $d_{1}$ of $1.1 v_{1}$ and $1.1 v_{2}$, respectively. Let $\widetilde{R}_{1}$ be the parallelogram with corners $0,8 \ell_{1}, 8 \ell_{1}+\ell_{2}$ and $\ell_{2}$. Since $v_{1}$ and $v_{2}$ have length at least $100 d_{1}$, this parallelogram is obtained from a translate of $R_{1}$ by first 'distorting it very slightly', and then making it significantly shorter and thicker. It is easy to check that a translate of $\widetilde{R}_{1}$ through a suitable lattice element has the property that any 'long' crossing of $R_{1}$ includes a 'long' crossing of $\widetilde{R}_{1}$, so, in $\mathcal{C}_{0}$, the probability that $\widetilde{R}_{1}$ has a 'long' black crossing is at least $c$. Define $\widetilde{R}_{2}$ from $R_{2}$ similarly. Since $\ell_{i}$ is close to $1.1 v_{i}$ and the vectors $v_{i}$ are not too short and are orthogonal, the area of $\widetilde{R}_{i}$ is (crudely) at least $8\left|v_{1}\right|\left|v_{2}\right| \geq 8 m n \geq 8 L_{0}^{2} \geq 8 N$ area $(D)$.

Applying a linear transformation mapping $\ell_{1}$ to $(1,0)$ and $\ell_{2}$ to $(0,1)$, we find that in the transformed model $\mathcal{C}_{0}^{\prime}$, with lattice of symmetries $\mathcal{L}^{\prime} \supset \mathbb{Z}^{2}$, the probability that the rectangle $R_{1}^{\prime}=[0,8] \times[0,1]$ has a black horizontal crossing 
is at least $c$, as is the probability that $R_{2}^{\prime}=[0,1] \times[0,8]$ has a black vertical crossing. Note that the image $D^{\prime}$ of our fundamental domain $D$ has diameter at most $1 / 10$, say, since $\left|\ell_{i}\right| \geq 100 d_{1} \geq 100 \operatorname{diam}(D)$ and the $\ell_{i}$ are close to orthogonal. Also, the area of $D^{\prime}$ is area $(D) \times \operatorname{area}\left(R_{1}^{\prime}\right) / \operatorname{area}\left(\widetilde{R}_{1}\right) \leq 1 / N$.

Let $\mathbb{T}$ be the torus obtained by taking the quotient of $\mathbb{R}^{2}$ by the lattice $10 \mathbb{Z}^{2}$ generated by $(10,0)$ and $(0,10)$. Since $10 \mathbb{Z}^{2} \subset \mathcal{L}^{\prime}$, we may choose $n=$ $100 /$ area $\left(D^{\prime}\right)$ translates of $D^{\prime}$ by elements of $\mathcal{L}^{\prime}$ so that their images in $\mathbb{T}$ cover $\mathbb{T}$ exactly once. This allows us to define a natural equivalent of $\mathcal{C}_{0}^{\prime}$ on $\mathbb{T}$; the corresponding probability measure may be seen as $\mathbb{P}_{0}^{n}$, where $n=100 / \operatorname{area}\left(D^{\prime}\right) \geq$ $100 N$. Moreover, given a rectangle that does not come 'close' to wrapping around the torus, the events that it has a horizontal black crossing in the plane or in the torus have the same probability.

Let $E$ be the event that some translate of $[0,8] \times[0,1]$ in $\mathbb{T}$ has a black horizontal crossing. Then $\mathbb{P}_{0}^{n}(E) \geq c$, and $E$ is a symmetric, increasing event in $\mathbb{P}_{0}^{n}$ in the sense of Theorem 7.2 Since $n \geq N$, from our choice (23) of $N$ and Theorem [7.2, we have $\mathbb{P}_{1}^{n}(E) \geq 1-\delta$. As in [4, let $R_{1}, \ldots, R_{100}$ be translates in $\mathbb{T}$ of the rectangle $[0,6] \times[0,2]$ arranged so that any 8 -by- 1 rectangle crosses some $R_{i}$ horizontally. Then we have $\mathbb{P}_{1}^{n}\left(\bigcup H_{\mathrm{b}}\left(R_{i}\right)\right) \geq 1-\delta$, so by Corollary 3.3 and our choice (22) of $\delta$ we have $\mathbb{P}_{1}^{n}\left(H_{\mathrm{b}}\left(R_{i}\right)\right) \geq 0.99$ for some $i$, and thus for all $i$.

Translating back to the plane, we see that in $\mathcal{C}_{1}^{\prime}$ (obtained from $\mathcal{C}_{1}$ by the linear transformation mapping $\mathcal{C}_{0}$ to $\mathcal{C}_{0}^{\prime}$ ), any 6-by-2 rectangle $R$ with corners at points of the lattice $\mathcal{L}^{\prime}$ has $\mathbb{P}\left(H_{\mathrm{b}}(R)\right) \geq 0.99$. The same argument shows that any 2-by-6 rectangle $R^{\prime}$ with lattice point corners has $\mathbb{P}\left(V_{\mathrm{b}}\left(R^{\prime}\right)\right) \geq 0.99$. From here it is very easy to prove that percolation occurs, using any of several standard methods; we shall give one example.

Note that if $S$ is a 2-by-2 square then, with $\mathbb{P}$ denoting the probability measure associated to $\mathcal{C}_{1}^{\prime}$, we have $\mathbb{P}\left(H_{\mathrm{b}}(S)\right) \geq \mathbb{P}\left(H_{\mathrm{b}}(R)\right) \geq 0.99$ and $\mathbb{P}\left(V_{\mathrm{b}}(S)\right) \geq$ $\mathbb{P}\left(V_{\mathrm{b}}\left(R^{\prime}\right)\right) \geq 0.99$. As in 4 (the third version of the proof of Theorem 10 there), let $G(R)$ be the event that $H_{\mathrm{b}}(R)$ holds and each of the two 2-by-2 'end squares' of $R$ has a black vertical crossing, and define $G\left(R^{\prime}\right)$ similarly. Then $\mathbb{P}(G(R))$, $\mathbb{P}\left(G\left(R^{\prime}\right)\right) \geq 1-3(1-0.99)=0.97$. Of course, the bound 0.97 here can be replaced by any constant less than 1 , although, as we shall see, 0.97 is more than good enough.

Considering a square grid of 6 -by-2 and 2-by-6 rectangles overlapping in 2by- 2 squares as in 4 , and taking a bond of $\mathbb{Z}^{2}$ to be open if $G(R)$ holds for the corresponding rectangle, one obtains a dependent bond percolation measure on $\mathbb{Z}^{2}$. Given sets $S$ and $T$ of bonds of $\mathbb{Z}^{2}$ separated by a distance (in the graph $\mathbb{Z}^{2}$ ) of at least 1 , the corresponding unions of rectangles are disjoint, and are separated in the plane by a distance of at least $2 \geq 1 / 10$. It follows that the states of the bonds in $S$ are independent of the sates of the bonds in $T$, i.e., the bond percolation measure is 1-independent. It is rather easy to see that any such measure in which each bond is open with high enough probability has an infinite open cluster with probability 1; see, for example, the general domination result of Liggett, Schonmann and Stacey [19. The best current bound on what 'high 
enough' means is due to Balister, Bollobás and Walters [2], who showed that all bond probabilities at least 0.8639 will do. Since $\mathbb{P}(G(R)), \mathbb{P}\left(G\left(R^{\prime}\right)\right) \geq 0.97$, we see that with probability 1 there is an infinite open cluster in $\mathbb{Z}^{2}$. Translating back, the definition of $G(R)$ ensures that we find a corresponding infinite black cluster in $\mathcal{C}_{1}^{\prime}$. Since $\mathcal{C}_{1}^{\prime}$ is simply a linear image of $\mathcal{C}_{1}$, it follows that $\mathcal{C}_{1}$ contains an infinite black cluster with probability 1 ; hence $\mathcal{H}(\mathbf{q})$ percolates, as required.

It remains to establish exponential decay of the volume in $\mathcal{H}(\mathbf{q})$ for $\mathbf{q} \prec \mathbf{p}$. But first note that with $\mathbf{q} \succ \mathbf{p}$ as above, and with the arbitrary constant 0.99 replaced by a suitable constant $a<1$, the argument in [5, Section 3] (again using locally-dependent percolation) shows that the dual of the model $\mathcal{C}_{1}^{\prime}$ exhibits exponential decay (of the volume). It follows that the dual $\mathcal{H}^{*}\left(\mathbf{q}^{*}\right)$ of $\mathcal{H}(\mathbf{q})$ exhibits exponential decay.

Given a self-dual model $\mathcal{H}(\mathbf{p})$ and $\mathbf{q} \prec \mathbf{p}$, the model $\mathcal{H}^{*}\left(\mathbf{p}^{*}\right)$ is self-dual (it is isomorphic to $\mathcal{H}(\mathbf{p}))$, and $\mathbf{q}^{*} \succ \mathbf{p}^{*}$. Applying the result above to $\mathcal{H}^{*}\left(\mathbf{p}^{*}\right)$ and $\mathcal{H}^{*}\left(\mathbf{q}^{*}\right)$, we see that the dual $\mathcal{H}(\mathbf{q})$ of $\mathcal{H}^{*}\left(\mathbf{q}^{*}\right)$ exhibits exponential decay, as required.

Suppose instead that $\mathcal{H}(\mathbf{p})$ is approximately self-dual. Then by definition there is a model $\mathcal{H}^{\prime}\left(\mathbf{p}^{\prime}\right)$ that is isomorphic to $\mathcal{H}(\mathbf{p})$, such that $\mathcal{H}^{*}\left(\mathbf{p}^{*}\right)$ and $\mathcal{H}^{\prime}\left(\mathbf{p}^{\prime}\right)$ are equivalent, in the sense that they may be coupled so that for any open path in either model there is a nearby open path in the other model. Recall also that we may take the isomorphism to be given by an isometry of the plane (plus a small 'distortion', if needed). Taking the colouring viewpoint, open paths are simply black paths. Now the notion of equivalence is not obviously preserved under taking duals, i.e., inverting the colouring. However, the condition for approximate self-duality is exactly that for every white path in $\mathcal{H}(\mathbf{p})$ (corresponding to a black path in $\left.\mathcal{H}^{*}\left(\mathbf{p}^{*}\right)\right)$, there is a nearby black path in $\mathcal{H}^{\prime}\left(\mathbf{p}^{\prime}\right)$, and vice versa. Since $\mathcal{H}(\mathbf{p})$ and $\mathcal{H}^{\prime}\left(\mathbf{p}^{\prime}\right)$ are isomorphic, $\mathcal{H}^{\prime}\left(\mathbf{p}^{\prime}\right)$ also satisfies this condition, so we may couple $\mathcal{H}^{\prime}\left(\mathbf{p}^{\prime}\right)$ and $\mathcal{H}(\mathbf{p})$ so that for every white path in $\mathcal{H}^{\prime}\left(\mathbf{p}^{\prime}\right)$ there is a nearby black path in $\mathcal{H}(\mathbf{p})$, and vice versa. In other words, approximate self-duality holds after interchanging black and white, i.e., $\mathcal{H}^{*}\left(\mathbf{p}^{*}\right)$ is approximately self-dual. From this point the argument for exponential decay is as in the self-dual case.

\section{On the critical surface}

In the bulk of this paper we have shown that any self-dual hyperlattice percolation model $\mathcal{H}(\mathbf{p})$ is 'critical' in the sense that if $\mathbf{q} \succ \mathbf{p}$ then $\mathcal{H}(\mathbf{q})$ percolates, while if $\mathbf{q} \prec \mathbf{p}$ then $\mathcal{H}(\mathbf{q})$ exhibits exponential decay.

As noted earlier, the model $\mathcal{H}(\mathbf{p})$ itself may or may not percolate. Here we show that, except for degenerate cases, it does not. Furthermore, we show that one has power-law decay of the radius, as expected. Let $v_{0}$ be any fixed vertex of $\mathcal{H}$; we write $v_{0} \rightarrow r$ for the event that there is an open path from $v_{0}$ to a vertex at distance at least $r$ from $v_{0}$.

Theorem 8.1. Let $\mathcal{H}(\mathbf{p})$ be a malleable self-dual hyperlattice percolation model. Then there are constants $0<a_{1}<a_{2}$ and $r_{0}$ such that $r^{-a_{2}} \leq \mathbb{P}\left(v_{0} \rightarrow r\right) \leq r^{-a_{1}}$ 
for all $r \geq r_{0}$.

Note that since we argue directly about properties of the self-dual case, we need to impose the technical condition of malleability defined in Definition 5.9. It seems likely that this can be weakened to non-degeneracy; the latter condition is used throughout the proofs in the previous sections, whereas malleability is only used at one point, where the need for it could perhaps be circumvented.

Proof. By Lemma 5.10, $\mathcal{H}(\mathbf{p})$ can be realized by a malleable independent lattice colouring $\mathcal{C}$. By Theorem 6.3 , $\mathcal{C}$ has the large rectangles property and hence, by Lemma 6.5, the large ellipses property.

Let $S$ and $T$ be the maps exhibiting self-duality, as described in Lemma 6.7 Let $L=100 \max \left\{d_{0}, d_{1}\right\}$, where $d_{0}$ is the constant in Lemma 4.2 and $d_{1}$ is the bound on $|S(x)-T(x)|$ from Lemma 6.7

The large ellipses property tells us that there are constants $c>0$ and $A_{0}$ such that for every $A \geq A_{0}$ there is an ellipse $E=E(A)$ centred on the origin with area $A$ and with both axes having length at least $L$ such that $\mathbb{P}\left(O_{\mathrm{b}}(E)\right) \geq c$. Let $N$ be an integer such that $(1-c)^{N}<c$.

For $i \geq 0$ let $E_{i}=E\left((10 N)^{i} A_{0}\right)$ be an ellipse as above with area $(10 N)^{i} A_{0}$. We claim that for each $i, T\left(E_{i}\right)$ fits inside a copy of $E_{i+1}$ scaled by a factor $1 / 2$. To see this, rotate and scale so that the ellipse $T\left(E_{i}\right)$ becomes a circle with diameter 1, and $E_{i+1}$ has horizontal major axis with length $a$ and vertical minor axis with length $b$. Our claim is exactly that $b \geq 2$. But if not, then $b \leq 2$ so, since area $\left(E_{i+1}\right)=10 N$ area $\left(E_{i}\right)=10 N$ area $\left(T\left(E_{i}\right)\right)$, we have $a \geq 5 N$. Writing $E^{0}$ for the annulus between an ellipse $E$ and the concentric ellipse $2 E / 3$, it is not hard to see that when $b \leq 2$ and $a \geq 5 N$ one can arrange $N$ disjoint copies of $T\left(E_{i}\right)$ to 'cross' the annulus $E_{i+1}^{0}$ as in Figure 8.1 Moreover, in the rescaled

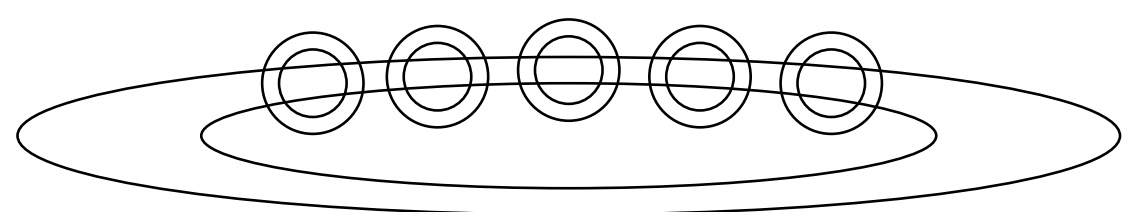

Figure 8.1: Circles 'crossing' an elliptical annulus. Depending on the height of the ellipse, the circles may or may not intersect the bottom half of the ellipse; this is irrelevant for the argument.

arrangement one can easily ensure that the circles are separated by a distance of at least $1 / 10$, say, and each 'crosses' $E_{i+1}^{0}$ even after any transformation moving points by a distance of at most $1 / 10$. Since lengths with original scale $\max \left\{d_{0}, d_{1}\right\}$ have transformed scale at most $1 / 100$, it follows that each image $S\left(E_{i}\right)$ crosses $E_{i+1}^{0}$, and the $S\left(E_{i}\right)$ are separated by a distance of at least $d_{0}$. But by self-duality each $S\left(E_{i}\right)$ contains a white path surrounding its centre with probability at least $c$, and these events are independent. Whenever one of these 
white paths is present, $O_{\mathrm{b}}\left(E_{i+1}\right)$ cannot hold. Hence $\mathbb{P}\left(O_{\mathrm{b}}\left(E_{i+1}\right)\right) \leq(1-c)^{N}<$ $c$, a contradiction.

Recalling from Lemma 6.7 that $T^{2}$ is either the identity or reflection in the origin, we see that $E_{i+2}^{0}$ surrounds $T\left(E_{i+1}^{0}\right)$ which surrounds $T\left(T\left(E_{i}^{0}\right)\right)=E_{i}^{0}$. Hence the annuli $E_{2 i}^{0}$ are disjoint. Moreover, since $1 / 2<2 / 3$ and all axes of all $E_{i}$ have length at least $L$, the annuli $E_{2 i}^{0}$ are separated by distances of at least $d_{0}$, and so meet disjoint sets of faces of $\mathcal{H}$. Now the shorter axis of $E_{i}$ has length at least $L \geq 1$, so its longer axis has length at most $(10 N)^{i} A_{0}$. It follows that for large $r$, any point at distance $r$ from the origin is outside $E_{k}$, where $k=\Theta(\log r)$. There can only be a white path starting at the origin and ending at least distance $r$ away if none of the events $O_{\mathrm{b}}\left(E_{2 i}\right), 2 i<k$, holds. Since these events are independent, this has probability at most $(1-c)^{\lfloor k / 2\rfloor}=\exp (-\Theta(\log r))$. Passing to the dual, this proves the upper bound on $\mathbb{P}\left(v_{0} \rightarrow r\right)$.

The lower bound is essentially immediate, with 1 as the exponent. Indeed, Lemma 4.4 tells us that if $R$ is an $r$-by- $r$ square, then either $\mathbb{P}\left(H_{\mathrm{b}}(R)\right) \geq 1 / 2$ or $\mathbb{P}\left(V_{\mathrm{w}}(R)\right) \geq 1 / 2$. Suppose first that $\mathbb{P}\left(H_{\mathrm{b}}(R)\right) \geq 1 / 2$. Then considering one of the $O(r)$ translates of our reference vertex $v_{0}$ within distance $O(1)$ of the left-hand side of $R$, by the union bound there is a translate of $v_{0}$ such that the probability that there is an open path starting at $v_{0}$ with length at least $r-O(1)$ is at least $O(1 / r)$.

If $\mathbb{P}\left(V_{\mathrm{w}}(R)\right) \geq 1 / 2$ then we apply the same argument in the dual, which is isomorphic to the original percolation model.

It is easy to see that the argument above extends to the approximately self-dual case. The key point is that there is enough 'elbow room' for small distortions of the paths considered not to matter.

It seems very probable that the general conjecture of Aizenman and Langlands, Pouliot and Saint-Aubin [18, concerning conformal invariance of the scaling limit of critical plane percolation will hold for all non-degenerate self-dual hyperlattice percolation models. However, this is likely to be very hard to prove. This conjecture asserts, among other things, that if $R$ is any rectangle, $\lambda R$ denotes its image under a dilation with scale-factor $\lambda$, and $H(R)$ denotes the event that $R$ has an open (or here, black) horizontal crossing, then for any fixed $R$, the $\operatorname{limit}_{\lim _{\lambda \rightarrow \infty}} \mathbb{P}(H(\lambda R))$ exists and lies strictly between 0 and 1 . Moreover, this limit should be given by Cardy's formula [9], after first applying a suitable linear transformation to the model.

This conjecture has been proved by Smirnov [28 for site percolation on the triangular lattice; this is essentially the only case known. For many other models, such as bond percolation on the square lattice, RSW-type theorems give the much weaker result that

$$
0<\liminf _{\lambda \rightarrow \infty} \mathbb{P}(H(\lambda R)) \leq \limsup _{\lambda \rightarrow \infty} \mathbb{P}(H(\lambda R))<1 .
$$

(This applies just as well to shapes other than rectangles.) Unfortunately, Theorem6.3. while strong enough to establish criticality, lacks the uniformity needed to prove (24), so we leave this as a conjecture. 
Conjecture 8.2. Let $\mathcal{H}(\mathbf{p})$ be a non-degenerate (approximately) self-dual hyperlattice percolation model. Then for any fixed rectangle $R$, the bounds (24) hold.

As far as we are aware, this conjecture is open even for the simple special case of inhomogeneous bond percolation on the square lattice, where each horizontal bond is open with probability $p$ and each vertical one with probability $1-p$, with the states of all bonds independent.

Acknowledgements. We are grateful to Robert Ziff for bringing the results of Scullard and himself to our attention: this paper started from an attempt to show that the self-duality they established in certain (quite general) cases does imply criticality. The last section of this paper was added in response to a question asked by Marek Biskup.

\section{References}

[1] M. Aizenman, H. Kesten and C. M. Newman, Uniqueness of the infinite cluster and related results in percolation, in Percolation Theory and Ergodic Theory of Infinite Particle Systems (Minneapolis, Minn., 1984-1985), Springer (1987), pp. 13-20.

[2] P. Balister B. Bollobás and M. Walters, Continuum percolation with steps in the square or the disc, Random Struct. Alg. 26 (2005), 392-403.

[3] B. Bollobás and O. Riordan, The critical probability for random Voronoi percolation in the plane is 1/2, Probability Theory and Related Fields 136 (2006), 417-468.

[4] B. Bollobás and O. M. Riordan, A short proof of the Harris-Kesten Theorem, Bull. London Math. Soc. 38 (2006), 470-484.

[5] B. Bollobás and O. Riordan, Sharp thresholds and percolation in the plane, Random Struct. Alg. 29 (2006), 524-548.

[6] B. Bollobás and O. Riordan, Percolation, Cambridge University Press, $2006, \mathrm{x}+323 \mathrm{pp}$.

[7] B. Bollobás and O. Riordan, Percolation on dual lattices with $k$-fold symmetry, Random Struct. Alg. 32 (2008), 463-472.

[8] J. Bourgain, J. Kahn, G. Kalai, Y. Katznelson and N. Linial, The influence of variables in product spaces, Israel J. Math. 77 (1992), 55-64.

[9] J.L. Cardy, Critical percolation in finite geometries, J. Phys. A 25 (1992), L201-L206.

[10] L. Chayes and H.K. Lei, Random cluster models on the triangular lattice, J. Stat. Phys. 122 (2006), 647-670. 
[11] L. Chayes and H.K. Lei, Cardy's formula for certain models of the bondtriangular type, Reviews in Math. Physics 19 (2007), 511-565.

[12] E. Friedgut and G. Kalai, Every monotone graph property has a sharp threshold, Proc. Amer. Math. Soc. 124 (1996), 2993-3002.

[13] G. Grimmett, Percolation, Second edition, Springer, 1999, xiv+444 pp.

[14] T.E. Harris, A lower bound for the critical probability in a certain percolation process, Proc. Cam. Philos. Soc. 56 (1960), 13-20.

[15] J. Kahn, G. Kalai and N. Linial, The influence of variables on boolean functions, Proc. 29th Annual Symposium on Foundations of Computer Science, 68-80, Computer Society Press, 1988.

[16] H. Kesten, The critical probability of bond percolation on the square lattice equals 1/2, Comm. Math. Phys. 74 (1980), 41-59.

[17] H. Kesten, Percolation Theory for Mathematicians, Birkhäuser, 1982, iv +423 pp.

[18] R. Langlands, P. Pouliot and Y. Saint-Aubin, Conformal invariance in twodimensional percolation, Bull. Amer. Math. Soc. (N.S.) 30 (1994), 1-61.

[19] T.M. Liggett, R.H. Schonmann and A.M. Stacey, Domination by product measures, Ann. Probab. 25 (1997), 71-95.

[20] G.A. Margulis, Probabilistic characteristics of graphs with large connectivity, Problemy Peredači Informacii 10 (1974), 101-108.

[21] M.V. Menshikov, Coincidence of critical points in percolation problems, Soviet Math. Dokl. 33 (1986), 856-859.

[22] L. Russo, A note on percolation, Z. Wahrsch. Verw. Gebiete 43 (1978), $39-48$.

[23] L. Russo, On the critical percolation probabilities, Z. Wahrsch. Verw. Gebiete $\mathbf{5 6}$ (1981), 229-237.

[24] C.R. Scullard, Exact site percolation thresholds using a site-to-bond transformation and the star-triangle transformation, Phys. Rev. E 73 (2006), 016107 [6 pages]

[25] M.R.A. Sedlock and J.C. Wierman, Equality of bond percolation critical exponents for pairs of dual lattices, Phys. Rev. E 79 (2009), 051119 [10 pages]

[26] P.D. Seymour and D.J.A. Welsh, Percolation probabilities on the square lattice, in Advances in graph theory (Cambridge Combinatorial Conf., Trinity College, Cambridge, 1977). Ann. Discrete Math. 3 (1978), pp. 227-245. 
[27] S. Sheffield, Random surfaces, Astérisque 304 (2005), vi+175 pp.

[28] S. Smirnov, Critical percolation in the plane: conformal invariance, Cardy's formula, scaling limits, Comptes Rendus de l'Académie des Sciences. Série I. Mathématique, 333 (2001), 239-244. Expanded version available at www.math.kth.se/ ${ }^{\text {stas/papers. }}$

[29] P.N. Suding and R.M. Ziff, Site percolation thresholds for Archimedean lattices, Phys. Rev. E 60 (1999), 275-283.

[30] M.F. Sykes and J.W. Essam, Some exact critical percolation probabilities for bond and site problems in two dimensions, Physical Review Letters 10 (1963), 3-4.

[31] J. C. Wierman, Bond percolation on honeycomb and triangular lattices, Adv. in Appl. Probab. 13 (1981), 298-313.

[32] J. C. Wierman, A bond percolation critical probability determination based on the star-triangle transformation, J. Phys. A 17 (1984), 1525-1530.

[33] J.C. Wierman and R.M. Ziff, Triangle-duality and equality of infinitely many bond percolation thresholds, preprint (2009). http://arxiv.org/abs/0903.3135v1

[34] F.Y. Wu, New critical frontiers for the Potts and percolation models, Physical Review Letters 96 (2006), 090602 [4 pages]

[35] R.M. Ziff, Generalized cell-dual-cell transformation and exact thresholds for percolation, Phys. Rev. E 73 (2006), 016134 [6 pages]

[36] R.M. Ziff and C.R. Scullard, Exact bond percolation thresholds in two dimensions, J. Phys. A: Math. Gen. 39 (2006), 15083-15090. 